\title{
Effects of Serotonin and Noradrenaline on Neuroplasticity and Excitability of The Primary Motor Cortex in Humans
}

\author{
Dissertation \\ zur Erlangung des humanwissenschaftlichen Doktorgrades \\ in der Medizin \\ der Georg-August-Universität Göttingen
}

vorgelegt von

Kuo, Hsiao-I

Aus

Taiwan

Goettingen, 2017 


\section{Doctoral Thesis Committee}

Prof. Dr. Nitsche, Michael (Supervisor)

Abteilung Klinische Neurophysiologie

Universitätsmedizin Göttingen

Robert-Koch-Straße 40

37075 Göttingen

Prof. Dr. Niklas, Andree (Thesis reviewer)

Georg August University Göttingen

Institut for sportmedizin

Sprangerweg 2

37075 Göttingen

Prof. Dr. Blumentritt, Siegmar (Thesis reviewer)

PFH Private Hochschule Goettingen

ZHT Zentrum fur Healthcare Technology

Robert-Koch-Strasse 40

37075 Goettingen 


\section{Statement of Originality}

I hereby declare that this thesis "Effects of Serotonin and Noradrenaline on Neuroplasticity and Excitability of The Primary Motor Cortex in Humans" was independently written and with no other sources and aids than quoted in the text, references and acknowledgements.

Goettingen, 10.01.2017

Kuo, Hsiao-I 



\section{Table of Contents}

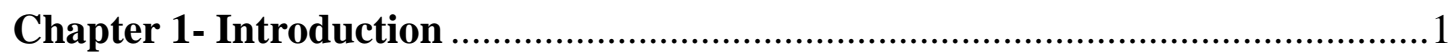

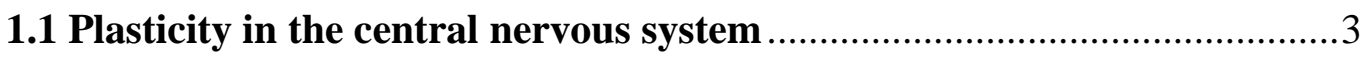

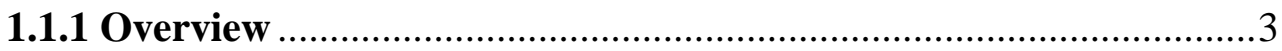

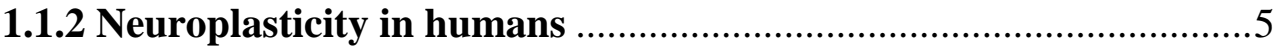

1.1.3 Motor system as a model for neuroplasticity in humans................5

1.2 Non-invasive brain stimulation in humans........................................

1.2.1 Transcranial magnetic stimulation (TMS) ...................................

1.2.2 Transcranial direct current stimulation (tDCS) .......................... 8

1.3 Pharmacological modulation of human cortical plasticity......................10

1.3.1 Serotonergic modulation of plasticity ........................................ 11

1.3.2 Noradrenergic modulation of plasticity ......................................12

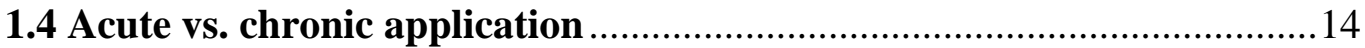

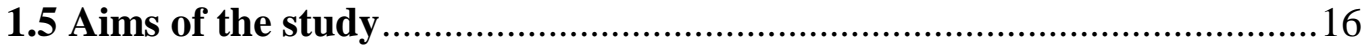

Chapter 2- Original articles and manuscripts...................................................... 17

2.1 Chronic enhancement of serotonin facilitates excitatory transcranial direct current stimulation-induced neuroplasticity ....................................... 18

2.2 Acute and chronic effects of noradrenergic enhancement on transcranial direct current stimulation (tDCS)-induced neuroplasticity in humans ........28

2.3 Acute and chronic noradrenergic effects on cortical excitability in

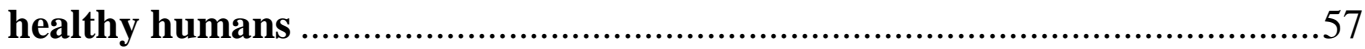

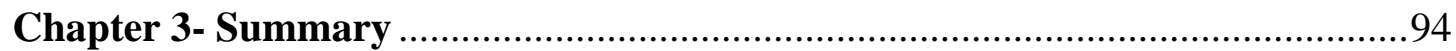

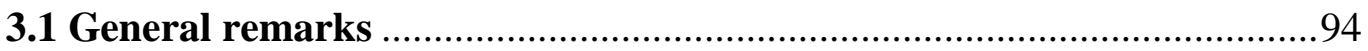

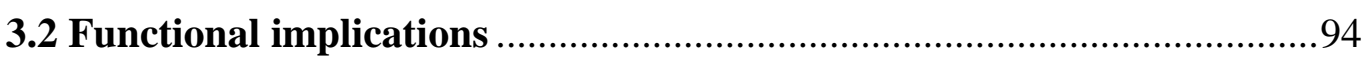

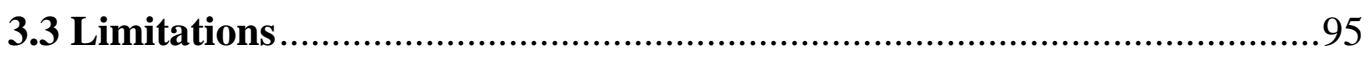

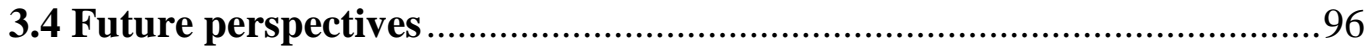

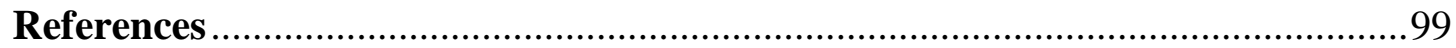

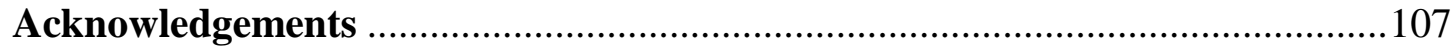

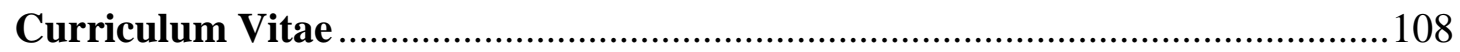




\section{Chapter 1- Introduction}

Neuroplasticity is the capacity of neural activity generated by an experience to modify neural circuit function and thereby modify subsequent thoughts, feelings, and behaviors (Criti \& Malenka, 2008). The best explored forms of plasticity are long-term potentiation (LTP) and long-term depression (LTD), that is, the strengthening or weakening of excitatory (or inhibitory) synapses (Criti \& Malenka, 2008). Recently, abnormal neuroplasticity has come increasingly into the focus as a correlate and pathological mechanism in many neurological and psychiatric diseases (e.g., stroke, Alzheimer's disease, Parkinson's disease, and depression) (Cooke \& Bliss, 2006). Therefore, modification of such pathological plasticity, or enhancing beneficial plasticity in these diseases, might be an interesting new therapeutic option (Kuo et al., 2014).

At the cellular level, plasticity is induced predominantly at glutamatergic and GABAergic synapses. The respective plasticity can however be altered by so-called neuromodulators, such as dopamine (DA), acetylcoline (Ach), serotonin (5-HT), and noradrenaline (NE) (Gu, 2002). These effects have so far been mainly studied in animal models. Typically, the role of neuromodulators in synaptic changes critically depends on the receptor subtypes, the concentration and kind of activity of the modulators, and their site of action. Among these neuromodulators, serotonin and noradrenaline received more attention recently since both of them are involved in diverse brain functions and also play important role in different psychiatric diseases (Ohashi et al., 2003; Straube \& Frey, 2003). A number of studies have shown that serotonin can enhance LTP (Kojic et al., 1997; Park et al., 2012) and block LTD (Normann et al., 2007). Activation of 5-HT receptors was further shown to convert 
LTD induction into LTP (Kemp \& Manahan-Vaughan, 2005). Furthermore, application of a selective serotonin reuptake inhibitor (SSRI) led to significantly enhanced LTP in the rat hippocampus, but the effect was present only after repeated application (Kemp \& Manahan-Vaughan, 2005). With regard to the noradrenergic system, noradrenaline has shown to facilitate LTP and block LTD (Katsuki et al., 1997; $\mathrm{Hu}$ et al., 2007). In addition, chronic administration of the selective noradrenaline reuptake inhibitor (NRI) reboxetine (RBX) restored spatial learning deficits and hippocampal synaptic plasticity in an animal model of depression (Bhagya et al., 2015).

Recently developed noninvasive brain stimulation protocols provide the opportunity to study LTP/LTD-like plasticity at the system level of the human brain (Cooke \& Bliss, 2006; Ziemann et al., 2008). Transcranial direct current stimulation (tDCS) is one of these stimulation protocols. It induces prolonged excitability changes in humans: Anodal stimulation increases and cathodal stimulation decreases cortical excitability (Nitsche \& Paulus, 2001; Nitsche et al., 2003a). Its primary mechanism of action is neural hyperpolarization accomplished by cathodal tDCS and subthreshold neural depolarization induced by anodal tDCS. Neuroplastic aftereffects are N-methyl-D-asparate (NMDA) and $\mathrm{Ca}^{2+}$-dependent and affected by neuromodulators, such as dopamine, acetylcholine, serotonin, and noradrenaline (Nitsche et al., 2003b; Nitsche et al., 2004; Kuo et al., 2007; Nitsche et al., 2009). Previous studies have found that dopaminergic and cholinergic activation affects plasticity in humans, which depends on dosage, the plasticity induction protocol, and the subtype of receptors (Nitsche et al., 2012).

The effects of serotonin and noradrenaline on plasticity in humans were explored in two recent experiments. A single dose of the SSRI citalopram enhanced both the 
amplitude and duration of the after effect of anodal tDCS, whereas it reversed the inhibition seen after cathodal tDCS to facilitation (Nitsche et al., 2009). Regarding noradrenaline, the monoamine reuptake inhibitor amphetamine enhanced duration of facilitation induced by anodal tDCS (Nitsche et al., 2004). In addition, previous studies suggested that acute application of selective NRI enhances cortical excitability in the human brain (Herwig et al., 2002). These results give us an initial insight how serotonin and noradrenaline affect neuroplasticity and cortical excitability in the human brain. Interestingly, clinical studies showed that it usually takes several weeks to obtain therapeutic effects of serotonergic and noradrenergic agents (Bezchilbnyk-Butler et al., 2000; Kasper et al., 2000). However, knowledge about the impact of chronic serotonergic and noradrenergic enhancement in humans on plasticity and excitability is limited at present.

In this project, we were interested to explore the impact of acute and chronic serotonergic and noradrenergic receptor activity enhancement on functional plasticity of the human brain. Furthermore, we investigated the acute and chronic effects of noradrenaline on cortical excitability in humans. The first chapter introduces basic mechanisms relevant for understanding the studies included in the thesis. The second chapter consists of the papers presenting the research results. The concluding chapter summarizes the main results of the studies and offers an outlook to future research in the field.

\subsection{Plasticity in the central nervous system}

\subsubsection{Overview}

One of the most fascinating properties of the mammalian brain is its plasticity; the 
ability of brain to reorganize its structure and function due to intrinsic or environmental demands (Criti \& Malenka, 2008). At the cellular level, long term potentiation (LTP) and long term depression (LTD) are the most widely studied neuroplastic mechanisms considered to be fundamental for learning and memory formation (Rioult-Pedotti et al., 2000; Cooke \& Bliss, 2006). These processes are most detailed studied at glutamatergic synapses, especially in the region of the hippocampus, but also in other cortical and subcortical areas (Malenka \& Bear, 2004). Plasticity of the glutamatergic system is accomplished primarily via calcium-permeable N-methyl-D-asparate (NMDA) receptors (Cooke \& Bliss, 2006). The induction of LTP is accomplished by activation of postsynaptic NMDA receptors and calcium-dependent protein kinases which results in the postsynaptic insertion of alpha-amino-3-hydroxy-5-methyl-4-isoxazolepropionate (AMPA) receptors (Criti \& Malenka, 2008). LTD is generated by moderate activation of NMDA receptors and another type of calcium-dependent enzymes which leads to the internalization of postsynaptic AMPA receptors (Criti \& Malenka, 2008). It is well known that increased postsynaptic intracellular calcium concentration is an important signal for the induction of LTP and LTD (Gu, 2002; Malenka \& Bear, 2004; Criti \& Malenka, 2008). High enhancement of intracellular calcium induces LTP, whereas low enhancement results in LTD (Lisman, 2001). The mechanisms of synaptic alteration are in accordance to the rules of Hebbian plasticity, characterized by longevity, input specificity and associativity, which state that learning and memory are based on modifications of synaptic strength among neurons (Martin et al., 2000). Moreover, these mechanisms are important for adaptive reorganization of cortical networks of the brain following physiological or pathological changes (Buonomano \& Merzenich, 1998). 


\subsubsection{Neuroplasticity in humans}

Despite the findings in animal models, direct evidence in humans has shown that neuroplastic alterations play a crucial role in the cerebral cortex (Cooke \& Bliss, 2006). Studies demonstrated that neural activity in ipsilesional and contralesional cortical areas are pathologically changed (Aydina et al., 2007) following injury in stroke patients (Levy et al., 2001; Hodics et al., 2006). However, these changes are converted after rehabilitation, and correlated to functional recovery (Johansen-Berg et al., 2010). Apart from stroke, pathological alterations of plasticity are increasingly explored as relevant factors in diverse neurological and psychiatric diseases, such as Parkinson's disease, Alzheimer disease and depression (Ueki et al., 2006; Normann et al., 2007). Not only in pathological states, but also in physiological conditions, such kinds of plasticity have been observed, for example in musicians (Pantev et al., 2001), mathematicians (Aydina et al., 2007), athletes (Park et al., 2009) and following motor practice (Ziemann et al., 2001). Therefore, exploring the mechanisms of neuroplasticity can improve our knowledge about fundamental mechanisms of brain physiology not only in health but also in disease.

\subsubsection{Motor system as a model for neuroplasticity in humans}

In humans, cortical excitability alterations as a sign of LTP/LTD-like plasticity is typically monitored by the amplitude of the motor-evoked potential (MEP), which is elicited by transcranial magnetic stimulation (TMS) of the motor cortex. For obtaining MEPs, small hand muscles are most often used because of the superficial position of their motor cortex representation, low thresholds for stimulation and relatively large representations. In all studies mentioned in the thesis, MEPs have 
been obtained from the abductor digiti minimi muscle (ADM). The MEP amplitude obtained by single pulse TMS is a measure of corticospinal excitability (Rothwell, 1993) that reflects the synaptic strength and the balance of excitatory and inhibitory inputs at the synapses of corticospinal neurons (Ziemann, 2003).

\subsection{Non-invasive brain stimulation in humans}

Recently, a number of non-invasive brain stimulation tools applicable in humans became available such as TMS, repetitive transcranial magnetic stimulation (rTMS), transcranial direct current stimulation (tDCS), and paired associative stimulation (PAS), which can further increase our understanding of cortical plasticity via monitoring cortical excitability, or controlled induction of plasticity. With these techniques, it has become possible to evaluate and influence cortical activity in awake, behaving humans. Here, we introduce TMS and tDCS, which are related to our current studies.

\subsubsection{Transcranial magnetic stimulation (TMS)}

TMS was introduced by Anthony Barker in 1985 (Barker et al., 1985). It is a safe and painless noninvasive brain stimulation technique to study neural activity in conscious humans (Kobayashi \& Pascual-Leone, 2003; Wagner et al., 2007). TMS uses a rapidly changing magnetic field to elicit electric currents running parallel to the cortical surface via electromagnetic induction. These brief pulsed magnetic fields painlessly pass through the skull and can create electric currents of sufficient magnitude in discrete brain regions to depolarize neurons. TMS activates both excitatory and inhibitory neuronal elements in the cerebral cortex (Kobayashi \& Pascual-Leone, 2003). Unlike electric stimulation, which excites cortical output 
neurons directly (Merton \& Morton, 1980), TMS is thought to stimulate these neurons indirectly via interneurons and can therefore elicit responses that reflect cortical excitability. The design of TMS consists of a main stimulator and a stimulating coil. In the studies discussed below, TMS is applied to the primary motor cortex (M1) for eliciting a response in the abductor digiti minimi (ADM) muscle. The resulting MEP is recorded via surface electromyography (EMG) (Rothwell, 1993). TMS can be delivered in the form of single, paired, and repetitive pulses; each of those protocols is applied for different purposes. In our studies, we applied single and paired pulse TMS, which we introduce in more detail below.

\subsubsection{Single-pulse TMS}

Single-pulse TMS provides information about corticospinal excitability by measuring variables such as motor thresholds (MTs) (including: active motor threshold (AMT), and resting motor threshold (RMT)), MEP amplitudes, and the input-output curve (I-O curve). MTs reflect neuronal membrane excitability and depend primarily on ion channel activity, as MTs are increased by voltage-gated sodium channel blockers, but not affected by drugs modulating gamma-aminobutyric acid (GABA)-ergic or glutamatergic transmission (Ziemann et al., 1996; Ziemann et al., 1998a). The I-O curve serves as an index of excitability of larger neuronal populations compared to MTs (Chen, 2000; Abbruzzese \& Trompetto, 2002). The I-O curve depends on neuronal membrane excitability, because its slope is decreased by sodium and calcium channel blockers. Furthermore, synaptic mechanisms are involved, as it is modulated by drugs influencing the GABAergic and glutamatergic system, especially with higher stimulation intensities (Boroojerdi et al., 1999; Di Lazzaro et al., 2003). 


\subsubsection{Paired-pulse TMS}

Paired-pulse is delivered at varying interstimulus intervals and stimulation intensities to elicit cortical inhibition or facilitation. Short-latency intracortical inhibition (SICI) and facilitation (ICF), motor cortex indirect waves (I-waves), and short-interval afferent inhibition (SAI) were monitored by paired-pulse TMS in our studies. For obtaining SICI-ICF, the subthreshold conditioning stimulus (determined as $70 \%$ of AMT) precedes the test stimulus. The test pulse was adjusted to achieve a baseline MEP of $\sim 1 \mathrm{mV}$ and readjusted during the respective stimulation protocols, if needed, to compensate for effects of global excitability changes on test-pulse amplitude. SICI is mainly controlled by glutamate and $\mathrm{GABA}_{\mathrm{A}}$ receptors and based on induction of inhibitory postsynaptic potentials (Ziemann et al., 1996; Liepert et al., 1997; Ziemann et al., 1998a). ICF is thought to reflect activity of GABAergic and predominantly glutamatergic systems (Ziemann, 2004). For I-wave facilitation, the TMS test stimulus precedes the conditioning stimulus (determined as $70 \%$ of RMT). I-waves are thought to be primarily controlled by GABA-related neuronal circuits (Ziemann et al., 1998a; Ziemann et al., 1998b). SAI combines peripheral and motor cortex stimulation. In this protocol, a suprathreshold electric pulse (width of $200 \mu \mathrm{s}$ and an intensity of $200 \%$ of the perceptual threshold) over the ulnar nerve precedes the motor cortex TMS test pulse. With SAI, it is possible to evaluate the cholinergic system in humans such as in patients with Alzheimer's disease (Di Lazzaro et al., 2006).

\subsubsection{Transcranial direct current stimulation (tDCS)}

This non-invasive brain stimulation tool was established in the 1950s and 1960s 
primarily in animals (Bindman et al., 1964b; Purpura \& Mcmurtry, 1965). According to these early experiments, it was shown that subthreshold direct current stimulation increases spontaneous neuronal activity if the anode is placed over the cortex, while reversal of electrode polarity, i.e. positioning of the cathode over the motor cortex, resulted in reduced activity (Bindman et al., 1964a; Purpura \& Mcmurtry, 1965). Later experiments revealed the excitability and activity changes can last for many hours after the end of stimulation for a few minutes, depend on protein synthesis (Gartside, 1968), intracellular cyclic adenosine monophosphate (cAMP) concentration (Hattori et al., 1990), and gene expression mediated by N-methyl-D-asparate (NMDA) receptors (Islam et al., 1995a; Islam et al., 1995b). Therefore, the after-effects depend on plasticity mechanism similar to those obtained in LTP and LTD induced by "classic" stimulation protocols (Islam et al., 1995a).

For application in humans, Nitsche and Paulus developed non-invasive transcranial application of weak direct currents to the human motor cortex, termed transcranial direct current stimulation (tDCS) (Nitsche \& Paulus, 2000, 2001). Anodal stimulation elicits neural depolarization which enhances cortical excitability, whereas cathodal tDCS results in neural hyperpolarization which diminishes cortical excitability (Nitsche \& Paulus, 2001; Nitsche et al., 2003a). Stimulation for some minutes results in respective neuroplastic effects, which depend on glutamatergic mechanisms and are calcium-dependent (Nitsche and Paulus, 2000, 2001; Nitsche et al., 2003a; Nitsche et al., 2005). Nevertheless, tDCS-induced plasticity differs from classical plasticity induction protocols, used primarily in vitro. The latter involve not tonic subthreshold, but pulsatile suprathreshold stimulation. Thus, mechanisms of plasticity might not be identical, although similarities like NMDA receptor dependency, and calcium dependency, are present (Liebtanz et al., 2002; Nitsche et al., 2003b). 
TDCS consists of applying prolonged, low-intensity electrical currents over the scalp -usually delivered by a small battery-driven constant current stimulator- by attaching electrodes (sizes employed about $25-35 \mathrm{~cm}^{2}$ ) with different polarities to the skin (Brunoni et al., 2012). To stimulate the primary motor cortex, typically one electrode is placed on the scalp over M1 and the other over the contralateral supraorbital area. The duration and strength of the after-effects of tDCS usually depend on stimulation intensity and duration (Nitsche \& Paulus, 2001; Nitsche et al., 2003a). In addition to the motor cortex, tDCS can also be applied to visual and somatosensory systems, and has been shown to modulate cognitive processes, when applied over respective target regions (Kuo et al., 2014; Shin et al., 2015; Balzarotti \& Colombo, 2016). TDCS protocols should state current strength, electrode size, stimulation duration, and electrode position to enhance comparability between studies (Nitsche et al., 2008).

\subsection{Pharmacological modulation of human cortical plasticity}

Neuromodulator systems (dopaminergic, cholinergic, serotoninergic and noradrenergic) have modulating roles on plasticity ( $\mathrm{Gu}, 2002)$. Activation of these systems is not necessary to induce plasticity, but these systems have the capacity to modify the amount and direction of plasticity induced. Generally, the impact of neuromodulators depends on several factors such as the type of receptor subtypes, the dosage of the substance, and the cortical background activity in the specific brain region. Neuromodulators may impact on cortical plasticity through facilitation or reduction of NMDA receptor-gated processes, beyond other mechanisms (Gu, 2002). Serotonin and noradrenaline both play crucial roles in diverse brain functions and are also correlated with various neurological and psychiatric disorders (Normann et al., 
2007; Marzo et al., 2009). Given the involvement of both transmitters in neuroplasticity, it is of great interest how pharmacological intervention will influence neurophysiologically induced plasticity.

\subsubsection{Serotonergic modulation of plasticity}

Serotonin (or 5-HT) is one of the most important neuromodulators in the central nervous system $(\mathrm{Gu}, 2002)$. It is also an important agent in diverse neuropsychiatric diseases such as depression. Serotonin has multiple receptor subtypes $\left(5-\mathrm{HT}_{1}, 5-\mathrm{HT}_{2}\right.$, 5- $\mathrm{HT}_{3}, 5-\mathrm{HT}_{4}, 5-\mathrm{HT}_{5}, 5-\mathrm{HT}_{6}$, and 5- $\mathrm{HT}_{7}$ receptors), which are linked to multiple signal transduction mechanisms and related to learning and memory formation in animals and humans (Geyer, 1996; Jacobs BL \& Formal, 1997; Bert et al., 2008). One important foundation for its effects might be its impact on neuroplasticity ( $\mathrm{Gu}, 2002)$. Animal experiments have shown that serotonin affects LTP and LTD in slice preparations. The direction of the effects depends on receptor subtypes, dosage of respective drugs, duration of 5-HT receptor activation, and site of action (Kojic et al., 1997; Mori et al., 2001; Ohashi et al., 2003; Kemp \& Manahan-Vaughan, 2005). In different studies, serotonin resulted in both LTP-enhancing and -abolishing effects (Kojic et al., 1997; Park et al., 2012). Regarding LTD, application of 5-HT agonists blocks LTD or even converts it into LTP, whereas 5-HT antagonists enhance LTD expression (Kemp \& Manahan-Vaughan, 2005). These results confirm that serotonin is involved in brain plasticity, but the specific effects are complex.

The effects of acute serotonin enhancement on motor cortical plasticity induced by tDCS and paired associative stimulation (PAS) were explored recently in healthy humans. In a foregoing study, a single dose of the selective serotonin receptor inhibitor (SSRI) citalopram enhanced both the amplitude and duration of the 
after-effects of anodal tDCS until the same evening of stimulation, and it reversed the excitability diminution seen after cathodal tDCS into facilitation (Nitsche et al., 2009). Likewise for PAS, acute application of citalopram enhanced PAS-induced LTP-like after-effects and abolished LTD-like PAS-induced after-effects (Batsikadze et al., 2013). These results show a prominent impact of serotonin on plasticity in humans. Similar effects were obtained for visual cortex plasticity (Normann et al., 2007). These effects on plasticity might partially explain the positive effects of SSRI on motor task performance and memory formation in healthy individuals as well as on the recovery processes in rehabilitation therapy after stroke (Loubinoux et al., 2002; Loubinoux et al., 2005; Acler et al., 2009).

\subsubsection{Noradrenergic modulation of plasticity}

Noradrenaline is a crucial neuromodulator in the central nervous system which increases excitability and thus affects learning and memory processes in animals and humans (Wang et al., 2011; Robinson, 2012). Similar to serotonin, the precise effect of noradrenaline on plasticity, as explored in animal models, is complex and depends on receptor subtype, concentration, and the site of action (Marzo et al., 2009). Specifically, noradrenaline affects the direction of LTP as well as LTD dependent on the activation of $\alpha$ - and $\beta$-adrenoreceptors (Kemp \& Manahan-Vaughan, 2008; Marzo et al., 2009). Pharmocological and receptor cloning studies have given rise to a further subdivision of this main grouping into $\alpha_{1}$ (divided into $\left.\alpha_{1 \mathrm{~A}}, \alpha_{1 \mathrm{~B}}, \alpha_{1 \mathrm{D}}\right), \alpha_{2}\left(\alpha_{2 \mathrm{~A}}, \alpha_{2 \mathrm{~B}}, \alpha_{2 \mathrm{C}}\right.$, $\left.\alpha_{2 \mathrm{D}}\right)$, and $\beta_{1}, \beta_{2}, \beta_{3}$ receptor subtypes $(\mathrm{Gu}, 2002)$. In general, activation of $\beta$-adrenoreceptors enhances LTP, whereas the activation of $\alpha$-adrenoreceptors reduces it (Marzo et al., 2009; Wojtowicz et al., 2010). For LTD, the effects of adrenoreceptors show conflicting results. With regard to $\beta$-adrenoreceptors, Kemp and 
Mahahan-Vaughan described an enhancement of LTD, whereas LTD was abolished in another study (Katsuki et al., 1997; Kemp \& Manahan-Vaughan, 2008). For $\alpha$-adrenoreceptors, $\alpha 2$ receptor activation reduces LTD, while $\alpha 1$ receptors enhance it (Nakadate et al., 2006). The underlying mechanism of noradrenaline on neuroplasticity might be that activation of adrenoreceptors results in the activation of various intracellular factors and modifications of membrane ion channel opening (Nakadate et al., 2006; Marzo et al., 2009). $\beta$-adrenoreceptors activation decreases potassium conductance and results in depolarization of postsynaptic neurons (Hass \& Konnerth, 1983). This results in enhancement of calcium currents into the intraneuronal compartment through NMDA receptors and voltage-dependent calcium channels, which is closely related to LTP induction (Heinbotham \& Dunwiddie, 1991). In contrast, $\alpha$-adrenoreceptors induce neuronal membrane hyperpolarization by opening of potassium channels, which inhibits voltage activated calcium currents, and might be related to LTD expression (Kirwood et al., 1999). Furthermore, $\alpha$-adrenoreceptors enhance GABA-induced inhibition in different cortical areas (Lei et al., 2007).

With regard to noradrenergic modulation of human brain plasticity, a foregoing study has shown that a single dose of the monoamine reuptake inhibitor amphetamine enhances the duration of the aftereffects of anodal tDCS (Nitsche et al., 2004). Furthermore, in accordance with the above-mentioned experiments in animal models, both, the aftereffects induced by anodal and cathodal tDCS, were reduced by a $\beta$-adrenergic receptor blocker (Nitsche et al., 2004). Methylphenidate, a noradrenaline-dopamine reuptake inhibitor, had however no effect on PAS-induced LTP-like plasticity, while PAS-induced LTP-like plasticity was abolished by an $\alpha 1$ receptor antagonist (Korchounov \& Ziemann, 2011). Taken together, the results of 
these studies suggest that the adrenergic system significantly impacts on plasticity in humans, which is in accordance with the results from animal studies (Marzo et al., 2009).

Growing evidence suggests that psychiatric diseases such as major depression are accompanied by compromised LTP, which can be re-installed by antidepressant treatment (Campell \& Macqueen, 2004; Castren, 2004). Patients with major depression show reduced LTP-like plasticity, as compared with healthy controls (Normann et al., 2007), and application of antidepressant agents can increase LTP-like plasticity. It was furthermore recently shown that chronic administration of the selective noradrenaline reuptake inhibitor (NRI) reboxetine (RBX) restored spatial learning deficits and hippocampal synaptic plasticity in an animal model of depression (Bhagya et al., 2015). For studies in humans, acute administration of RBX improves cognition and motor performance in healthy and depressed subjects (Ferguson et al., 2003; Wang et al., 2009). In summary, selective NRI might at least partially exert their treatment effects by enhancing LTP-like plasticity in depression, and therefore improve learning and cognition. With regard to therapeutic application, apart from depression, RBX has been shown to improve maximum grip power and finger tapping frequency in stroke patients, which might also be caused by noradrenaline-dependent modulation of neuroplasticity (Wang et al., 2011). Thus, NRI might have a potential for clinical application in various neurological and psychiatric diseases accompanied by pathological alterations of plasticity. However, knowledge about the impact of noradrenergic enhancement on neuroplasticity in humans on plasticity is limited at present.

\subsection{Acute vs. chronic application}


In clinical studies, it is well known that maximal effects of serotonergic and noradrenergic agents are obtained after weeks or even months of treatment (Bezchilbnyk-Butler et al., 2000; Kasper et al., 2000), suggesting that longer-term adaptive changes contribute to therapeutic efficacy. Animal studies suggest that both single and repeated treatments with SSRI can enhance synaptic efficacy, but only repeated treatments significantly facilitated the induction of LTP compared to placebo medication (Ohashi et al., 2002). Another difference was observed after acute and chronic administration of SSRIs: a down-regulation of the brain-derived neurotrophic factor (BDNF) gene expression after $4 \mathrm{~h}$ (acute) and an up-regulation after 14 days (chronic) of application of the agent in the rat hippocampus (Coppell et al., 2003). Besides, chronic administration of the selective noradrenaline reuptake inhibitor (NRI) reboxetine results in a greater net increase of extracellular noradrenalin and dopamine compared to an acute dose (Page \& Lucki, 2002). Another NRI, desipramine improved cognitive functions in both acutely and chronically treated rats (Lapiz et al., 2007). In an human functional magnetic resonance imaging (fMRI) study, chronic administration of selective serotonin reuptake inhibitor (SSRI) induced a significant hypoactivation of the primary sensorimotor cortex, but an hyperactivation after single dose treatment (Loubinoux et al., 2005). Furthermore, recent TMS studies have shown different modulation of the input-output curve (I-O curve) and intracortical facilitation (ICF) after chronic administration of SSRI compared to single dose application (Gerdelat-Mas et al., 2005). These results imply that cortical excitability might be modulated in different ways according to the duration of treatment. One candidate mechanism, which can however not explain all results, might be desensitization or downregulation of receptors. The specific interaction between dosage, plasticity and serotonergic/noradrenergic activity in the 
human brain is so far not systematically explored.

\subsection{Aims of the study}

The purpose of this work is to investigate the impact of serotonergic and noradrenergic neuromodulation on human brain physiology, especially cortical excitability, and plasticity, in larger detail. According to the literature, acute administration of the SSRI citalopram enhances facilitatory plasticity. Based on the above-mentioned study results, it might be speculated that chronic effects of citalopram might differ physiologically and functionally. Moreover, we are interested in the underlying mechanisms, i.e., modulatory effects on glutamatergic plasticity, and on the plasticity of the serotonergic system itself. Therefore, in the first study, we explored the impact of chronic application of the citalopram on plasticity induced by tDCS in healthy humans. Likewise for noradrenlaine, studies showed that acute administration affected neuroplasticity in humans. However, knowledge about the chronic effect of noradrenaline on neuroplasticity in humans is still missing. Thus, for the second study, we investigated the acute and chronic effects of selective NRI reboxetine on neuroplasticity in healthy subjects. Beyond its impact on plasticity, recent studies have shown that noradrenaline impacts also on cortical excitability in humans. Nevertheless, most of the studies so far were conducted in a single dose design. It cannot be excluded that also for cortical excitability, acute and chronic effects of noradrenergic enhancement differ. Thus for the third study, we explored the acute and chronic effects of noradrenaline on cortical excitability in humans. The knowledge we aim to gain via these studies might further help to optimize the manipulation of cortical plasticity for clinical therapeutic interventions. 


\section{Chapter 2- Original articles and manuscripts}

This chapter contains two published articles and one submitted manuscript. The first study focused on chronic effects of serotonin on neuroplasticity in healthy humans. The second and third study investigated acute and chronic noradrenergic effects on neuroplasticity as well as cortical excitability in healthy humans.

I. Kuo HI, Paulus W, Batsikadze G, Jamil A, Kuo MF, Nitsche MA (2016) Chronic Enhancement of serotonin facilitates excitatory transcranial direct current stimulation-induced neuroplasticity. Neuropsychopharmacology 41:1223-1230 (Published)

II. Kuo HI, Paulus W, Batsikadze G, Jamil A, Kuo MF, Nitsche MA (2016) Acute and chronic effects of noradrenergic enhancement on transcranial direct current stimulation (tDCS)-induced plasticity in humans. The Journal of Phsyiology 7:1-10 (Published)

III. Kuo HI, Paulus W, Batsikadze G, Jamil A, Kuo MF, Nitsche MA. Acute and chronic noradrenergic effects on cortical excitability in healthy humans. (in revision) 


\subsection{Chronic enhancement of serotonin facilitates excitatory transcranial direct current stimulation-induced neuroplasticity}

Neuroplasticity, which is the dynamic structural and functional reorganization of central nervous system, is the foundation of various cognitive, motor, and behavioral processes in humans. In the past decades, it became increasingly clear that pathological alteration of neuroplasticity is involved in various neuropsychiatric diseases. Therefore, modification of such pathological plasticity might be a promising therapeutic opportunity. Indeed, studies have found that distress disrupts neuroplasticity, while antidepressant treatment, including serotonin enhancement via selective serotonin reuptake inhibitor (SSRI), produces opposing effects in animal models and humans (Henn \& Vollmayer, 2004; Normann et al., 2007). In a foregoing study, a single dose of the SSRI citalopram enhanced both the amplitude and duration of the after-effects of anodal transcranial direct current stimulation (tDCS) until the same evening of stimulation, and it reversed the excitability diminution seen after cathodal tDCS into facilitation (Nitsche et al., 2009). Likewise for paired associative stimulation (PAS), acute application of citalopram enhanced long term potentiation (LTP)-like PAS-induced after-effects and abolished long term depression (LTD)-like PAS-induced after-effects (Batsikadze et al., 2013). Therefore, SSRI treatment may exert a therapeutic effect via modulation of brain plasticity. Clinically it usually takes several weeks to obtain therapeutic effects via SSRI (Bezchilbnyk-Butler et al., 2000). Thus it can be speculated that the physiological effects of acute and chronic serotonin enhancement differ. However, the effect of chronic administration of SSRIs on neuroplasticity in humans has not yet been explored, and the underlying mechanism is unclear. Here we explored the impact of chronic SSRI citalopram application on tDCS-induced neuroplasticity in healthy humans in a randomized, double-blinded, 
crossover design. The N-methyl-D-asparate (NMDA) receptor antagonist (dextromethorphan) was also applied to test the role of citalopram in modulating glutamatergic plasticity. 12 subjects received anodal or cathodal tDCS over the primary motor cortex combined with placebo medication. Afterwards, they took citalopram (20 mg/day) for 35 days, during which four additional plasticity induction sessions were applied (citalopram combined with dextromethorphan or placebo + anodal/ cathodal tDCS). Plasticity was monitored by motor evoked potentials with transcranial magnetic stimulation. Chronic application of citalopram increased and prolonged the LTP-like plasticity induced by anodal tDCS for over 24 hours, and converted cathodal tDCS-induced LTD-like plasticity into facilitation. These effects were abolished by the NMDA receptor antagonist dextromethorphan. Thus chronic serotonergic enhancement results in a strengthening of LTP-like glutamatergic plasticity, which might partially explain the therapeutic impact of SSRIs in depression and other neuropsychiatric diseases. 


\title{
Chronic Enhancement of Serotonin Facilitates Excitatory Transcranial Direct Current Stimulation-Induced Neuroplasticity
}

\author{
Hsiao-I Kuo', Walter Paulus', Giorgi Batsikadze', Asif Jamil', Min-Fang Kuo² and Michael A Nitsche ${ }^{*, 1,2,3}$ \\ 'Department of Clinical Neurophysiology, University Medical Center, Georg-August-University, Göttingen, Germany; ${ }^{2}$ Department of Psychology \\ and Neurosciences, Leibniz Research Centre for Working Environment and Human Factors, Dortmund, Germany; ${ }^{3}$ Department of Neurology, \\ University Medical Hospital Bergmannsheil, Bochum, Germany
}

\begin{abstract}
Serotonin affects memory formation via modulating long-term potentiation (LTP) and depression (LTD). Accordingly, acute selective serotonin reuptake inhibitor (SSRI) administration enhanced LTP-like plasticity induced by transcranial direct current stimulation (tDCS) in humans. However, it usually takes some time for SSRI to reduce clinical symptoms such as anxiety, negative mood, and related symptoms of depression and anxiety disorders. This might be related to an at least partially different effect of chronic serotonergic enhancement on plasticity, as compared with single-dose medication. Here we explored the impact of chronic application of the SSRI citalopram (CIT) on plasticity induced by tDCS in healthy humans in a partially double-blinded, placebo (PLC)-controlled, randomized crossover study. Furthermore, we explored the dependency of plasticity induction from the glutamatergic system via $\mathrm{N}$-methyl-D-aspartate receptor antagonism. Twelve healthy subjects received PLC medication, combined with anodal or cathodal tDCS of the primary motor cortex Afterwards, the same subjects took CT (20 mg/day) consecutively for 35 days. During this period, four additional interventions were performed (CIT and PLC medication with anodal/cathodal tDCS, CIT and dextromethorphan ( $150 \mathrm{mg}$ ) with anodal/cathodal tDCS). Plasticity was monitored by motor-evoked potential amplitudes elicited by transcranial magnetic stimulation. Chronic application of CIT increased and prolonged the LTP-like plasticity induced by anodal tDCS for over $24 \mathrm{~h}$, and converted cathodal tDCS-induced LTD-like plasticity into facilitation. These effects were abolished by dextromethorphan. Chronic serotonergic enhancement results in a strengthening of LTP-like glutamatergic plasticity, which might partially explain the therapeutic impact of SSRIs in depression and other neuropsychiatric diseases.

Neuropsychopharmacology (2016) 4I, 1223-1230; doi:10.1038/npp.2015.270; published online 23 September 2015
\end{abstract}

\section{INTRODUCTION}

Serotonin (or 5-HT), one of the most important neuromodulators in the central nervous system, is related to learning and memory formation in animals and humans (Bert et al, 2008; Jacobs and Formal, 1997). It is also an important agent in depression. One important foundation for its effects might be its impact on neuroplasticity (Gu, 2002). Animal experiments have shown that serotonin can affect long-term potentiation (LTP) and long-term depression (LTD) in slice preparations. The direction of the effects depends on receptor subtypes, dosage of respective drugs, duration of 5-HT receptor activation, and site of action (Kemp and Manahan-Vaughan, 2005; Kojic et al, 1997; Mori et al, 2001). Both LTP-enhancing and -abolishing

*Correspondence: Professor Dr MA Nitsche, Department Psychology and Neuroscience, Leibniz Research Centre for Working Environment and Human Factors, Ardeystrasse 67,44139 Dortmund, Germany, Tel: +49 55 I 39 9571, Fax +49 551 39 8126, E-mail: nitsche@ifado.de Received 9 April 2015; revised 12 July 2015; accepted 17 August 2015; accepted article preview online 2 September 2015 effects were described in different studies (Kojic et al, 1997; Park et al, 2012). Furthermore, application of 5-HT agonists blocks LTD or even converts it into LTP, whereas 5-HT antagonists enhance LTD expression (Kemp and ManahanVaughan, 2005). Concerning human studies, it was shown that selective serotonin reuptake inhibitor (SSRI) enhance LTP-like plasticity of late visual-evoked potentials in healthy subjects, whereas LTD-like plasticity was converted into facilitation (Normann et al, 2007). Similar effects were observed for motor cortex plasticity (Batsikadze et al, 2013; Nitsche et al, 2009). Moreover, several studies have demonstrated that serotonin enhancers can improve learning and memory formation, and motor functions in healthy individuals, as well as functional outcome in depression and stroke patients (Acler et al, 2009; Brunoni et al, 2013), ie, functional effects that involve neuroplasticity. These results confirm that serotonin is involved in brain plasticity, but the specific effects are complex.

The effects of acute serotonin enhancement on motor cortical plasticity induced by transcranial direct current stimulation (tDCS) and paired associative stimulation (PAS) 
were explored recently in healthy humans. These noninvasive brain stimulation tools induce prolonged cortical excitability changes (Nitsche et al, 2003b; Nitsche and Paulus, 2000, 2001; Stephan et al, 2000; Ziemann et al, 2008). tDCS induces non-focal plasticity via the primary mechanism of tonic subthreshold modulation of resting membrane potentials (Nitsche et al, 2007, 2008). Cathodal tDCS results in neural hyperpolarization and anodal tDCS elicits neural depolarization. For motor cortex stimulation, anodal tDCS enhances, whereas cathodal tDCS diminishes cortical excitability (Nitsche et al, 2003a; Nitsche and Paulus, 2000, 2001). In contrast, PAS induces focal and synapse-specific plasticity of the respective target neurons, via combined activation of the motor cortex and peripheral afferents. PAS shares some features with spike timingdependent plasticity. The direction of plasticity depends on the synchronous or asynchronous activation of the target neurons (Stephan et al, 2000). Both stimulation protocols induce LTP-/LTD-like plasticity of the glutamatergic system, which is $N$-methyl-D-aspartate (NMDA) receptor- and calcium-dependent (Liebetanz et al, 2002; Nitsche et al, 2003b; Stephan et al, 2000). In a foregoing study, a single dose of the SSRI citalopram (CIT) enhanced both the amplitude and duration of the after-effects of anodal tDCS until the same evening of stimulation, and it reversed the excitability diminution seen after cathodal tDCS into facilitation (Nitsche et al, 2009). Likewise for PAS, acute application of CIT enhanced LTP-like PAS-induced after-effects and abolished LTD-like PAS-induced aftereffects (Batsikadze et al, 2013). These results show a prominent impact of serotonin on plasticity in humans.

For clinical implications in psychiatric diseases, pathologically altered plasticity, especially compromised LTP, recently came into the focus of attention as a potentially important pathophysiological mechanism in major depression. Distress disrupts neuroplasticity, whereas antidepressant treatment, including serotonin enhancement via SSRI, produces opposing effects in animal models (Henn and Vollmayr, 2004; Holderbach et al, 2007). Accordingly, plasticity is disturbed in patients with depression, but restituted by SSRI application (Normann et al, 2007). Thus, SSRI treatment may exert a therapeutic effect via modulation of brain plasticity. Although an delayed clinical impact of antidepressant medication is usually assumed, these might act faster than previously thought. New data suggest that the maximum improvement of clinical symptoms occurs during the first 2 weeks after initiation of antidepressant medication (Stassen and Angst, 2012). However, a single dosage of SSRI has usually no prominent clinical effects. Thus, it can be speculated that the physiological effects of acute and chronic serotonin enhancement differ in a similar manner. However, the effect of chronic administration of SSRIs on neuroplasticity in humans has not yet been explored. We hypothesized that chronic application of the SSRI CIT would enlarge the neuroplastic excitability enhancement, which is induced by anodal tDCS, whereas cathodal tDCS-induced inhibitory plasticity should be converted into excitation, in accordance with the results obtained by acute administration of CIT. Based on the superior clinical effects induced by repeated administration of SSRI, we hypothesized that chronic application should enhance LTP-like plasticity, ie, induce a larger and/or longer-lasting excitability enhancement

Neuropsychopharmacolog compared with the acute medication condition. We additionally combined SSRI administration with an NMDA receptor antagonist to demonstrate that in accordance with its neuromodulatory effects, serotonin enhancement does not induce plasticity itself, but modulates plasticity of the glutamatergic system. Accordingly we hypothesized that the NMDA receptor antagonist would prevent plasticity induction via tDCS also in the presence of SSRI.

\section{MATERIALS AND METHODS}

\section{Subjects}

Twelve right-handed healthy subjects participated in the experiment (five men and seven women, aged $27.5 \pm 4.01$ years). Inclusion criteria were age between 18 and 50 years, no history of chronic or acute neurological, psychiatric, or medical diseases, no family history of epilepsy, no present pregnancy, no cardiac pacemaker, no previous surgery involving implants in the head (cochlear implants, aneurysm clips, brain electrodes), and absent acute or chronic medication or drug intake, including nicotine. All participants gave written informed consent. The study was approved by the ethics committee of the University of Göttingen, and conforms to the Declaration of Helsinki.

\section{Transcranial Direct Current Stimulation}

We used a battery-driven constant current stimulator (NeuroConn GmbH, Ilmenau, Germany) with a maximum output of $4.5 \mathrm{~mA}$. Two saline-soaked surface sponge electrodes $\left(35 \mathrm{~m}^{2}\right)$ were applied to deliver the current. One electrode was positioned over the motor cortex representation area of the right abductor digiti minimi muscle (ADM), and the other electrode was located above the right orbit. tDCS was administered with a current strength of $1 \mathrm{~mA}$ for $13 \mathrm{~min}$ (anodal tDCS) or $9 \mathrm{~min}$ (cathodal tDCS). These stimulation protocols induce prolonged excitability changes in the human motor cortex: anodal stimulation increases and cathodal stimulation decreases cortical excitability for $\sim 1 \mathrm{~h}$ after stimulation (Nitsche et al, 2003a; Nitsche and Paulus, 2001).

\section{Pharmacological Interventions}

Two hours before the start of each experimental session, $20 \mathrm{mg}$ CIT with placebo (PLC), $20 \mathrm{mg}$ CIT with $150 \mathrm{mg}$ dextromethorphan (DMO), or equivalent PLC medication only were administered orally to the subjects. The maximum plasma level is achieved $\sim 2 \mathrm{~h}$ after oral intake of these substances, and the respective doses are sufficient to elicit prominent effects in the central nervous system (Bezchilbnyk-Butler et al, 2000). The chosen dosage of CIT is identical with that applied in our foregoing experiments (Batsikadze et al, 2013; Nitsche et al, 2009). The chosen dosage of DMO abolished tDCS-induced plasticity in other experiments (Liebetanz et al, 2002; Nitsche et al, 2003b, 2004). 


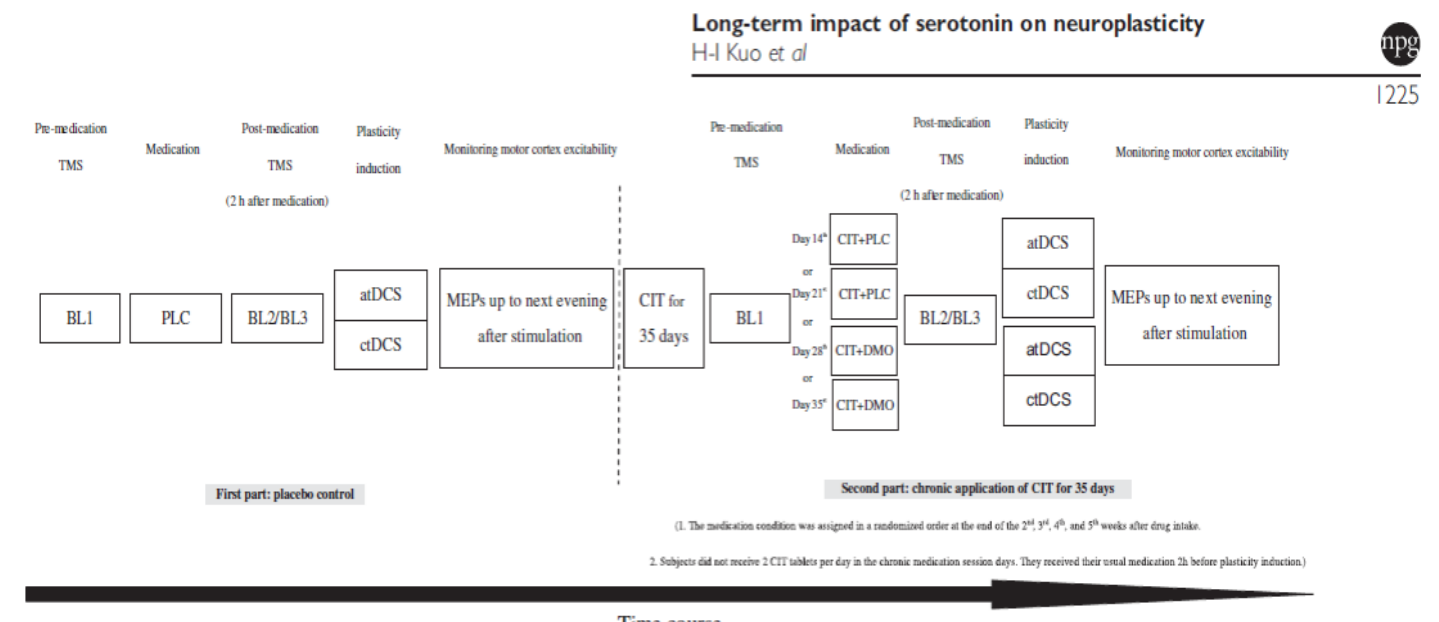

Time course

Figure I Experimental course of the present study. The study was conducted in two parts. For the first part, subjects received placebo medication (PLC) with cathodal or anodal tDCS. For the second part, the same subjects took $20 \mathrm{mg}$ CIT consecutively for 35 days. During this period, the other four sessions were conducted (CT and PLC with anodal/cathodal tDCS, CIT and $150 \mathrm{mg}$ dextromethorphan (DMO) with anodal/cathodal tDCS). For each session, first transcranial magnetic stimulation (TMS) was applied over the left motor cortical representation area of the right abductor digiti minimi muscle (ADM) with an intensity to elicit motor-evoked potentials (MEPs) with a peak-to-peak amplitude of on average I mV (BLI). Two hours after intake of the medication, a second baseline (BL2) was determined to control for a possible influence of the drug on cortical excitability and adjusted if necessary (BL3). Afterwards, transcranial direct current stimulation (tDCS) was applied and MEPs were recorded immediately after stimulation. Further TMS measurements were conducted in the evening of the same day (SE), next moming (NM), next noon (NN), and next evening (NE).

\section{Monitoring of Motor Cortical Excitability}

Cortical excitability was monitored by peak-to-peak amplitudes of motor-evoked potentials (MEPs) induced by transcranial magnetic stimulation (TMS) of the motor cortical representation of the right ADM. Single-pulse TMS was conducted by a Magstim 200 magnetic stimulator (Magstim Company, Whiteland, Dyfed, United Kingdom) connected with a figure-of-eight magnetic coil (diameter of one winding $=70 \mathrm{~mm}$, peak magnetic coil $=2.2 \mathrm{~T}$ ). The coil was held tangentially to the skull, with the handle pointing backwards and laterally at an angle of $45^{\circ}$ to the mid-sagittal plane. Electromyographic recording was obtained from the right $\mathrm{ADM}$ with $\mathrm{Ag}-\mathrm{AgCl}$ electrodes attached in a belly-tendon montage. Signals were filtered $(2 \mathrm{~Hz}$ to $2 \mathrm{kHz})$, amplified, and then stored on computer via a Power 1401 data acquisition interface (Cambridge Electronic Design, Cambridge, United Kingdom). TMS intensity was adjusted to elicit baseline MEPs of averaged $1 \mathrm{mV}$ peak-to-peak MEP amplitude and was kept constant for the post-stimulation assessment unless adjusted (see below).

\section{Experimental Procedures}

The experiment was conducted in a partially blinded (subjects were blinded for all stimulation and medication conditions, and the experimenter was blinded for the medication conditions in the second part), complete crossover, and PLC-controlled design. Each volunteer participated in all experimental sessions (six sessions per subject). The experimental sessions were carried out in randomized order and separated by 1 week to avoid cumulative drug or tDCS effects. A specific sequence of experimental sessions was randomly assigned to each subject, which differed for all participants. The study was separated in two parts. For the first part, subjects received PLC medication combined with cathodal or anodal tDCS before chronic CIT medication was started. In the second part, participants received CIT $(20 \mathrm{mg} /$ day) consecutively for 35 days. During this period, the other four sessions (CIT and PLC with anodal/cathodal tDCS, CIT and dextromethorphan with anodal/cathodal tDCS) were conducted in randomized order at the end of the second, third, fourth, and fifth week after the start of chronic drug intake. At the day of the respective stimulation session, the participants were seated in a comfortable chair with head and arm rest. TMS was applied over the left motor cortical representational area of the right ADM where it produced consistently the largest MEPs in the resting muscle (optimal site). The intensity of the TMS stimulus was adjusted to elicit MEPs with a peak-to-peak amplitude of on average $1 \mathrm{mV}$ (baseline 1). Two hours after intake of the medication (CIT (the usual once-daily dosage) plus dextromethorphan or PLC), a second baseline was recorded to monitor the possible influence of the drug on cortical excitability (baseline 2) and make the experimental design comparable to that of Nitsche et al (2009). The TMS intensity was adjusted to result in baseline MEP amplitudes of $1 \mathrm{mV}$ when necessary (baseline 3). Afterwards, tDCS was performed. Immediately after $\mathrm{tDCS}, 25 \mathrm{MEPs}$ were recorded at the time points of $0,5,10,15,20,25,30,60,90$, and $120 \mathrm{~min}$, and then again the same evening (SE: between $6 \mathrm{pm}$ and $7 \mathrm{pm}$ ), next morning (NM: between 9 am and $10 \mathrm{am})$, next noon (NN: between 12 am and $1 \mathrm{pm}$ ), and next evening (NE: between $6 \mathrm{pm}$ and $7 \mathrm{pm}$; Figure 1).

\section{Data Analysis and Statistics}

Individual MEP amplitude means were calculated for each time bin, including baseline 1,2, and 3 and post-stimulation time points, separately for each stimulation/medication 
Table I MEP Amplitudes and Stimulation Intensity Before and After Citalopram Administration

\begin{tabular}{lllrrrr}
\hline Stimulation & TMS parameter & Medication condition & Baseline I & Baseline 2 & Baseline 3 & p value \\
\hline Anodal tDCS & MEP & PLC & $0.97 \pm 0.1$ & $0.98 \pm 0.01$ & $0.99 \pm 0.01$ & 0.728 \\
& & CIT+PLC & $1.09 \pm 0.05$ & $1.00 \pm 0.05$ & $0.98 \pm 0.01$ & 0.103 \\
& \% MSO & CIT+DMO & $1.03 \pm 0.07$ & $1.00 \pm 0.09$ & $1.03 \pm 0.09$ & 0.266 \\
& PLC & $52 \pm 8.09$ & $52 \pm 8.09$ & $53.38 \pm 9.18$ & 0.166 \\
& CIT+PLC & $53.23 \pm 9.18$ & $53.23 \pm 9.18$ & $53.38 \pm 9.18$ & 0.485 \\
Cathodal tDCS & CT+DMO & $53.75 \pm 8.93$ & $53.75 \pm 8.93$ & $54.08 \pm 9.25$ & 0.305 \\
& MEP & PLC & $0.98 \pm 0.1$ & $0.97 \pm 0.06$ & $0.99 \pm 0.05$ & 0.786 \\
& CIT+PLC & $1 \pm 0.06$ & $0.94 \pm 0.18$ & $0.98 \pm 0.04$ & 0.266 \\
& CIT+DMO & $0.98 \pm 0.08$ & $0.99 \pm 0.05$ & $1.00 \pm 0.14$ & 0.815 \\
& PLC & $54.25 \pm 8.77$ & $54.25 \pm 8.77$ & $54.33 \pm 9.06$ & 0.795 \\
& CIT+PLC & $53.08 \pm 9.28$ & $53.08 \pm 9.28$ & $53.75 \pm 9.29$ & 0.166 \\
& CIT+DMO & $54 \pm 9.28$ & $54 \pm 9.28$ & $54.42 \pm 9.17$ & 0.096 \\
\hline
\end{tabular}

Shown are the mean MEP amplitudes \pm SD and stimulation intensity (percentage of maximum stimulator output, \%MSO) mean \pm SD of baseline I, 2, and 3. The intensity of TMS was determined to elicit MEPs with peak-to-peak amplitude of $\sim \mathrm{ImV}$ (baselinel). A second baseline (baseline 2) was recorded $2 \mathrm{~h}$ after medication intake to determine the effect of the drug on cortical excitability and adjusted if necessary (baseline 3). Student's t-tests revealed no significant differences between conditions $(P>0.05)$.

Table 2 Results of the Repeated Measures ANOVA and ANCOVA

\begin{tabular}{|c|c|c|c|c|c|c|}
\hline \multirow[b]{2}{*}{ Factor } & \multicolumn{3}{|c|}{ ANOVA } & \multicolumn{3}{|c|}{ ANCOVA } \\
\hline & d.f. & $\mathbf{F}$ & $P^{\prime}$ & d.f. & $\mathbf{F}$ & $p$ \\
\hline Drug & 2 & 23.894 & $<0.001^{\mathrm{a}}$ & I & 156.69 & $<0.001^{\mathrm{a}}$ \\
\hline tDCS & I & 1.709 & 0.218 & 1 & 8.973 & $0.003^{\mathrm{a}}$ \\
\hline Time course & 14 & 4.372 & $<0.001^{\mathrm{a}}$ & 14 & 5.572 & $<0.001^{\mathrm{a}}$ \\
\hline Drug $\times$ tDCS & 2 & 40.447 & $<0.001^{\mathrm{a}}$ & I & 42.781 & $<0.001^{\mathrm{a}}$ \\
\hline Drug $\times$ time & 28 & 3.703 & $<0.001^{\mathrm{a}}$ & 14 & 0.558 & 0.987 \\
\hline tDCS $\times$ time & 14 & 3.467 & $<0.001^{\mathrm{a}}$ & 14 & 3.315 & $<0.001^{\mathrm{a}}$ \\
\hline Drug $\times$ tDCS $\times$ time & 28 & 11.467 & $<0.001^{\mathrm{a}}$ & 14 & 0.953 & 0.502 \\
\hline Order & & & & 3 & 2.561 & 0.11 \\
\hline
\end{tabular}

Abbreviation: d.f, degrees of freedom.

asignificant results at $p<0.05$.

combination. The post-intervention MEPs were normalized and are given as ratios of the third baseline.

A repeated-measure analysis of variance (ANOVA) for the time bins up to the next evening measurement after tDCS was performed with the within-subject factors time course (time bins up to the next evening after stimulation), drug condition (PLC, CIT with PLC, CIT with DMO), stimulation type (anodal and cathodal tDCS), and the dependent variable MEP. The Mauchly test of sphericity was conducted, and the Greenhouse-Geissser correction was applied when necessary. In case of significant results of the ANOVA, post hoc comparisons were performed using Student's $t$-tests (paired samples, two-tailed, $p<0.05$, not corrected for multiple comparisons) to determine whether the MEP amplitudes before and after tDCS differed in each intervention condition and whether those differences depend on drug condition. Furthermore, we performed an ANCOVA for the chronic medication condition with order as co-variate to rule out systematic effects of order of conditions on the results. To explore whether medication modified baseline MEPs, additional tests (Student's $t$-tests, $p<0.05$ ) for comparison of baseline 1 and 2 were performed. To explore if baseline MEP, and the TMS intensity needed to obtain baseline MEP (percentage of maximal stimulator output) differed between tDCS and medication conditions, the respective baseline values were compared via Student's $t$-tests.

\section{RESULTS}

All subjects tolerated tDCS and medication without adverse events. No subjects dropped out due to side effects of medication or $\mathrm{tDCS}$. The average baseline MEP values and percentage of maximal stimulator output did not significantly differ between groups $(P>0.05$, Student's paired, two-tailed $t$-test). The peak-to-peak amplitude of the baseline MEPs was not significantly affected by medication between first and second baseline values $(P>0.05$, Student's paired, two-tailed $t$-test; Table 1).

The ANOVA revealed significant main effects of drug and time, drug $\times$ stimulation, drug $\times$ time, stimulation $\times$ time, and drug $\times$ stimulation $\times$ time interactions (Table 2). This is due to the following pattern of results: in the PLC condition, anodal and cathodal tDCS significantly increased or decreased cortical excitability, respectively, until $90 \mathrm{~min}$ after the end of stimulation. Interestingly, the excitabilityenhancing effects of anodal tDCS developed with a delay of $15 \mathrm{~min}$ after the end of tDCS, and also the excitabilitydiminishing effects of cathodal stimulation became significant not immediately, but $5 \mathrm{~min}$ after stimulation. Chronic application of CIT enlarged the MEP amplitudes significantly vs PLC medication and extended the duration of the facilitation induced by anodal tDCS until the evening of the day after $\mathrm{tDCS}$, and thus for more than $24 \mathrm{~h}$, whereas it turned cathodal tDCS-induced LTD-like into LTP-like plasticity (Figure 2). The latter effects, however, vanished

Neuropsychopharmacology 

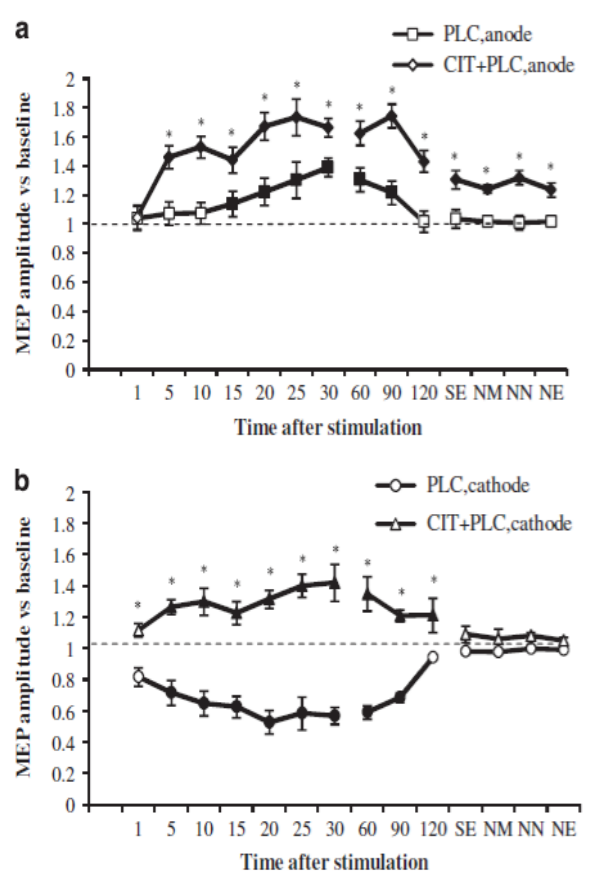

Figure 2 Impact of chronic serotonin enhancement on transcranial direct current stimulation (tDCS)-induced motor cortex plasticity. Shown are baseline-standardized MEP amplitudes after plasticity induction by anodal) cathodal tDCS under placebo (PLC) or citalopram with placebo (CIT+PLC) conditions up to the next evening of the post-stimulation day. (a) In the placebo medication condition (square), anodal tDCS induced a significant excitability enhancement for up to $90 \mathrm{~min}$ after stimulation. Citalopram (diamond) enhanced and prolonged these excitability enhancements unt next evening. (b) In the placebo medication condition (circle), cortical excitability was significantly reduced after cathodal tDCS for $90 \mathrm{~min}$, whereas citalopram (triangle) converted the inhibitory effect into facilitation. Error bars indicate SEM. Filled symbols indicate significant differences of post-stimulation MEP amplitudes from respective baseline values; asterisks indicate significant differences between the drug and placebo medication conditions at the same time points (Student's $t$-test, two-tailed, paired samples, $p<0.05)$. NE, next evening; NM, next morning, NN, next noon; SE, same evening.

120 min after tDCS. When combined with the NMDA receptor antagonist DMO, the after-effects generated by anodal and cathodal tDCS were absent (Figure 3). In this case, post-tDCS MEPs did not differ from baseline values, but did significantly differ from MEP amplitudes after cathodal and anodal tDCS combined with PLC medication.

The ANCOVA conducted for the chronic medication conditions did not result in a significant effect of order $(p=0.11 ;$ Table 2$)$.

\section{DISCUSSION}

The results of the present study show that chronic application of SSRI in healthy humans increased and extended the duration of the facilitation induced by anodal tDCS for more than $24 \mathrm{~h}$ after intervention, whereas it turned cathodal tDCS-induced inhibition into facilitation. These findings support recent concepts that the impact on neural
Long-term impact of serotonin on neuroplasticity $\mathrm{H}-\mathrm{l}$ Kuo et al
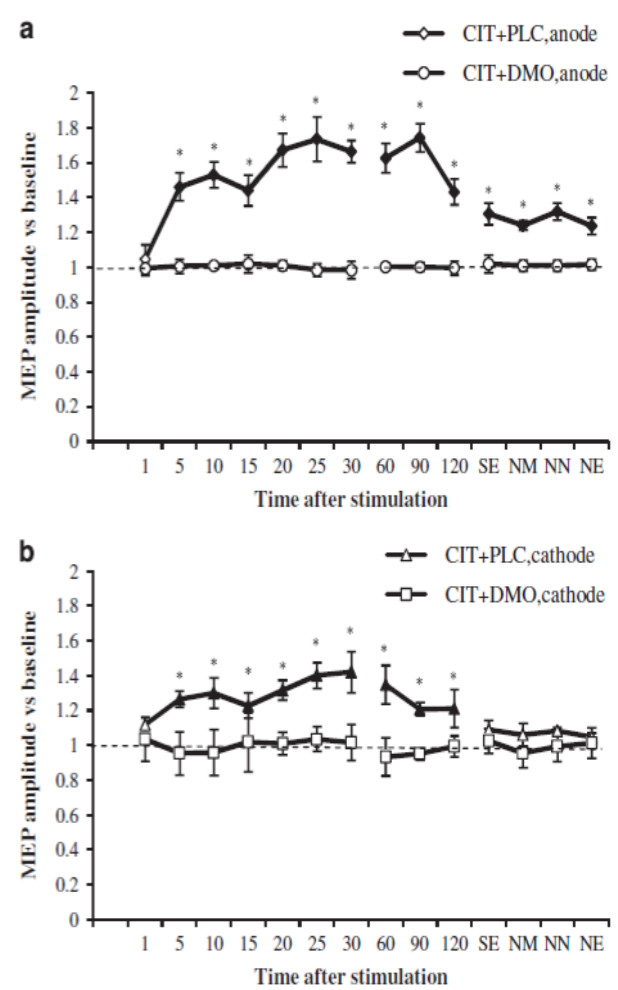

Figure 3 NMDA receptor block abolishes serotonin-dependent tDCS induced plasticity enhancements. Shown are the mean \pm SEM MEP amplitudes versus baseline across time following plasticity induction by anodal or cathodal tDCS for CIT with PLC (CT+PLC) and CIT with DMO (CTT+DMO) conditions. (a) DMO (circle) eliminated after-effects following anodal tDCS under CIT (diamonds). (b) DMO (square) likewise abolished the after-effects following cathodal tDCS under citalopram (triangle). Error bars indicate SEM. Filled symbols indicate significant differences of poststimulation MEP amplitudes from respective baseline values; asterisks indicate significant differences between two conditions at the same time points (Student's $t$-test, two tailed, paired samples, $p<0.05$ ). NE, next evening, NM, next morning $N N$, next noon; SE, same evening.

plasticity is relevant for therapeutic effects of serotonergic enhancement (Holderbach et al, 2007; Pittenger and Duman, 2007). The LTP-like effects of anodal tDCS lasted longer than $24 \mathrm{~h}$ after intervention. This duration exceeds that induced by single dose SSRI application in a foregoing study relevantly. Although direct comparability between study results is limited because of different participant groups, this might be a hint for better effects of chronic SSRI application on plasticity, which might-given the relevance of compromised LTP for depression-at least partially explain a need for repeated application of SSRI to exert antidepressant effects. DMO, a NMDA-antagonist, prevented both anodal and cathodal tDCS-induced after-effects, indicating that chronic SSRI effects are NMDA-receptor dependent, and are compatible with a neuromodulatory, but not plasticity-driving function of serotonin.

Our results extend previous data obtained in humans. Acute application of SSRI resulted in enhancement and prolongation of anodal tDCS-induced LTP-like plasticity and 
conversion of cathodal tDCS-induced LTD- into LTP-like plasticity (Nitsche et al, 2009). Furthermore, a single dose of SSRI strengthened excitatory PAS-induced, whereas it abolished inhibitory PAS-induced after-effects (Batsikadze et al, 2013). Likewise, application of SSRI increased facilitatory plasticity of late visual-evoked potentials and converted inhibitory plasticity into facilitation in healthy individuals (Normann et al, 2007). Thus, the impact of SSRI on plasticity is not restricted to single cortical areas, and seems not to be as qualitatively different between plasticity induction protocols, as it is the case for other neuromodulators like dopamine (Kuo et al, 2008; Monte-Silva et al, 2010; Thirugnanasambandam et al, 2011).

Our results also comply with some of the animal studies. Repeated application of SSRI can enhance LTP (Kojic et al, 1997; Mori et al, 2001). In addition, chronic application of the SSRI fluvoxamine prevented stress-induced facilitation of LTD and increased LTP both in stressed and non-stressed animals (Holderbach et al, 2007). Activation of 5-HT receptors was also shown to block LTD or further convert LTD induction into LTP (Kemp and Manahan-Vaughan, 2005; Normann and Clark, 2005). However, serotonin enhancement or activation of serotonergic receptors via specific agonists resulted in divergent effects in other studies. In some studies, serotonin or 5-HT receptor activation reduced or abolished LTP (Huang and Kandel, 2007; Kojima et al, 2003). This might be explained by the activation of different 5-HT receptor subtypes, the concentration of 5-HT agonists and antagonists, and different brain sites of action (Mori et al, 2001; Staubli and Otaky, 1994). To explore the reasons for these inconsistent results and to learn more about the impact of serotonin on neuroplasticity in humans, future studies should consider specific serotonergic receptor subtypes, apply different 5-HT-receptor agonists and antagonists, and explore the impact of different dosages, which might be a relevant factor for the strength and direction of effects, as seen for other neuromodulators, which have nonlinear effects on plasticity (Frensnoza et al, 2014; Monte-Silva et al, 2010).

Mechanistically, the impact of CIT on tDCS-generated plasticity can be explained by the impact of serotonin on calcium influx through NMDA receptors and voltage-gated calcium channels (Normann and Clark, 2005; Reiser et al, 1989). CIT reduces membrane potassium conductance and hereby enhances neuronal depolarization, which will lead to enhanced calcium influx via the above-mentioned channels (Panicker et al, 1991). Increased postsynaptic intracellular calcium concentration is an important signal for the induction of long-term synaptic plasticity (Criti and Malenka, 2008; Gu, 2002). High enhancement of intracellular calcium induces LTP, whereas low enhancement results in LTD (Lisman, 2001). As the after-effects of tDCS are NMDA receptor- and calcium-dependent (Liebetanz et al, 2002; Nitsche et al, 2003b), serotonergic enhancement might have strengthened the excitability enhancement induced by anodal tDCS and the conversion of LTD-like to LTP-like plasticity in case of cathodal tDCS via an enhancement of calcium influx. This assumption is supported by the fact that block of NMDA receptors, which reduces calcium influx, prevented plasticity induction via tDCS under CIT. Other mechanisms, such as reduction of serotonin autoreceptor density by CIT, or indirect effects by its modulatory effect on other transmitters and neuromodulators, cannot be ruled out, however, at present (Consolo et al, 1994; Wood and Wren, 2008). Which specific serotonin receptors are involved in these mechanisms is unclear. 5-HT2 and 5-HT4 receptors might be candidates. The 5-HT2 receptor stimulates intracellular calcium release, whereas the 5-HT4 receptor modulates calcium conductance, which induces LTP as well as depotentiation (Kulla and Manahan-Vaughan, 2002; Reiser et al, 1989). Concerning to the after-effects of tDCS, activation of the 5-HT2 receptor facilitates the induction of NMDA receptor-dependent LTP in the rat visual cortex (Kojic et al, 1997), and 5-HT4 receptor activation leads to expression on LTD in hippocampal slices (Kemp and Manahan-Vaughan, 2005). Given the LTP-like plasticityenhancing and LTD-like plasticity-abolishing effects of CIT in our study, the 5-HT2 receptor might be the more probable candidate.

In the present study, we aimed to investigate the effects of chronic serotonin enhancement on neuroplasticity in the human motor cortex. In summary, our results show that the SSRI CIT enhances and prolongs facilitatory plasticity, and converts inhibitory plasticity into facilitation. These findings add important information to our understanding of the mechanisms of consolidation of neuroplasticity in the human cortex. The modulatory action of SSRIs could also explain their positive effects on learning and memory in humans (Savaskan et al, 2008). In stroke and depression, reduced facilitatory and enhanced inhibitory plasticity have been described (Foy et al, 1987; Schaechter, 2004). SSRIs might reduce clinical symptoms in these diseases by antagonizing this imbalance.

tDCS is increasingly applied for treatment of neuropsychiatric diseases (Kuo et al, 2014), and in many cases it is used to induce LTP-like plasticity for therapeutic effects. Given the strengthening effect of CIT on the after-effects of tDCS, combining tDCS with SSRI might be a promising venue to enhance its clinical impact. In accordance, it was shown recently that combined tDCS and SSRI treatment had a superior impact on major depression, compared both of the interventions alone or PLC treatment (Brunoni et al, 2013). Likewise, SSRIs might be suited to strengthen tDCS effects in other diseases such as Alzheimer's disease, Parkinson's disease, or motor rehabilitation after stroke. Future studies should explore these possibilities and include functional outcomes to assess the agonistic effects of brain stimulation and SSRI therapy. Beyond tDCS, a variety of stimulation methods with different plasticity mechanisms can be used to evaluate the effects of SSRI on plasticity in humans. For example, PAS shares some characteristics with spike timing-dependent plasticity, which is assumed to be closely related to learning processes, which might be relevant for depression, especially with regard to therapeutic aspects (Stephan et al, 2000; Ziemann et al, 2008). Furthermore, plasticity induction of the dorsolateral prefrontal cortex with this stimulation protocol could be relevant for our understanding of the pathophysiological foundation of this disease, because this cortical area is closer related to respective symptoms of the disease as compared with the primary motor cortex, which was explored in the present study (Rajji et al, 2013).

Some limitations of the present study should be taken into consideration. First, we performed the PLC medication tDCS

Neuropsychopharmacology 
interventions before the start of long-term SSRI medication, thus the study was conducted in a partially blinded design, as described above. However, we did not tell subjects if they received real or PLC medication in the acute and chronic medication condition, furthermore we did not tell them about the respective tDCS condition. As participants described no side effects of medication, and tDCS polarity is not discernable, we assume that subjects were blinded. Second, we did not compare acute and chronic effects of serotonin enhancement in identical participants to restrict the number of sessions per participant. Consequently, comparability of effects between groups is limited. Nevertheless, taking into account the very similar duration of the after-effects under PLC-medication in both studies, comparability should be given at least to a certain degree. Third, participants had not the exact identical duration of CIT intake in the respective chronic medication conditions (medication duration between 14 and 35 days). To rule out systematic effects of order of conditions on the results, we conducted an ANCOVA for the chronic medication condition with order as co-variate. As the results show no significant impact of order, we think that pooling of data is justified. Fourth, this study was conducted in healthy young humans, and the primary motor cortex served as model for plasticity. One-to-one transferability of the results to participants with other characteristics (eg, patients with major depression) cannot be taken for granted, because basal state of brain activity and excitability will differ between groups, which might impact on $\mathrm{tDCS}$ effects. Moreover, it is currently unclear to what degree motor cortex plasticity results are transferable to other areas, which are more relevant for depression, such as the dorsolateral prefrontal cortex. However, as also motor cortex plasticity is reduced in major depression, but recovers along with reduction of depression symptoms (Player et al, 2014), M1 plasticity is assumed to be a feasible, although not ideal model for plasticity in major depression.

To our knowledge, this is the first pharmacological tDCS study evaluating effects of chronic pharmacological treatment on neuroplasticity in healthy participants. This paradigm can be used for other substances in future as well, in which different effects of acute and chronic medication are assumed, eg, for benzodiazepines. Our findings indicate that CIT shifts tDCS-induced plasticity into a facilitatory direction. This impact of serotonin on plasticity may be a relevant neurophysiological foundation of the effects of SSRIs in depressed patients. The results also suggest that modulation of brain plasticity via long-term SSRI application might be a promising pathway to treat patients with neurological deficits or psychiatric diseases who suffer from compromised plasticity.

\section{FUNDING AND DISCLOSURE}

The authors declare no conflict of interest.

\section{ACKNOWLEDGMENTS}

WP is a member of Advisory Boards of GSK, UCB, Desitin. MAN is a member of the Advisory Board of Neuroelectrics. H-IK is supported by the government scholarship, Republic of China (Taiwan). This study was supported by the BMBFproject, "Netzwerk psychische Erkrankungen", grant 01EE1403C. M-FK, H-IK, AJ, and GB received no financial support or compensation from any individual or corporate entity over the past 3 years for research or professional service and there are no personal financial holdings that could be perceived as constituting a potential conflict of interest.

\section{REFERENCES}

Acler M, Robol E, Fiaschi A, Manganotti P (2009). A double blind placebo RCT to investigate the effcts of serotonergic modulation on brain excitability and motor recovery in stroke patients. J Neurol 256: 1152-1158.

Batsikadze G, Paulus W, Kuo M, Nitsche M (2013). Effect of serotonin on paired associative stimulation-induced plasticity in the human motor cortex. Neuropsychopharmacology 38: 2260-2267.

Bert B, Fink H, Rothe J, Walstab J, Bonisch H (2008). Learning and momory in 5-HT(1A)-receptor mutant mice. Behav Brain Res 195: 78-85.

Bezchilbnyk-Butler K, Aleksic I, Kennedy S (2000). Citalopram- a review pf pharmachological and clinical effects. J Psychiatry Neurosci 25: 241-254.

Brunoni A, Valiengo L, Baccaro A, Zanao T, Oliveira Jd, Goulart A (2013). The sertraline vs electrical current therapy for treating depression clinical study: results from a factorial, randomized, controlled trial. JAMA Psychiatry 70: 1-9.

Consolo S, Arnaboldi S, Giogi S, Russi G, Ladinsky H (1994). 5-HT4 receptor stimulation facilitates acetylcholine release in rat frontal cortex. Neuroreport 5: 1230-1232.

Criti A, Malenka R (2008). Synaptic plasticity: multiple foms, functions, and mechanisms. Neuropsychopharmachology 33: $18-41$.

Foy M, Stanton M, Levine S, Thompason R (1987). Behavioral stress impairs long-term potentiation in rodent hippocampus. Behav Neural Biol 48: 138-149.

Frensnoza S, Paulus W, Nitsche MA, Kuo MF (2014). Nonlinear dose-dependent impact of D1 receptor activation on motor cortex plasticity in humans. J Neurosci 34: 2744-2753.

Gu Q (2002). Neuromodulatory transmitter systems in the cortex and their role cortical plasticity. Neuroscience 111: 815-835.

Henn FA, Vollmayr B (2004). Basic pathophysiological mechanisms in depression: what are they and how might they affect the course of the illness? Pharmacopsychiatry 37 (Suppl 2): S152-S156.

Holderbach R, Clark K, Moreu J, Bischofberger J, Norman C (2007). Enhanced long-term synaptic depression in an animal model of depression. Biol Psychiatry 62: 373-380.

Huang Y, Kandel E (2007). 5-Hydroxytryptamine induces a protein kinase a mitogen-dependent protein kinase-mediated and macromolecular synthesis-dependent late phase of long-term potentiation in the amagdala. J Neurosci 27: 3111-3119.

Jacobs BL, Formal C (1997). Serotonin and motor activity. Curr Opin Neurobiol 7: 820-825.

Kemp A, Manahan-Vaughan D (2005). The 5-hydroxytryptamine4 receptor exhibits frequnecy-dependent properties in synaptic plasticity and behavioral metaplasticity in the hippocampal CA1 region in vivo. Cereb Cortex 15: 1037-1043.

Kojic L, Gu Q, Douglas R, Cynader M (1997). Serotonin facilitates synaptic plasticity in kitten visual cortex: an in vitro study. Brain Res DEv Brain Res 101: 299-304.

Kojima T, Matsumoyo M, Yogashi H, Tachibana K, Kemmotsu O, Yoshioka M (2003). Fluvoxamine suppress the long-term potentiation in the hippocampal CA1 field of anesthetized 
rats: an effect medisted via 5-HT 1 A receptors. Brain Res 959: 165-168.

Kulla A, Manahan-Vaughan D (2002). Mosulation by serotonin 5-HT(4) receptors of long-term potentiation and depotentiation in the dentate gyrus of freely mobing rats. Cereb Cortex 12: 150-162.

Kuo MF, Paulus W, Nitsche MA (2008). Boosting focally-induced brain plasticity by dopamine. Cereb Cortex 18: 648-651.

Kuo MF, Paulus W, Nitsche MA (2014). Therapeutic effects of noninvasive brain stimulation with direct currents (tDCS) in neuropsychiatric diseases. Neuroimage 15: 948-960.

Liebetanz D, Nitsche M, Tergau F, Paulus W (2002). Pharmacological approach to synaptic and membrane mechanisms of DC-induced neuroplasticity in man. Brain 125: 2238-2247.

Lisman J (2001). Three Ca2+ levels affect plasticity differently: the

LTP zone, the LTD zone and no man's land. J Physiol 532: 285.

Monte-Silva K, Liebetanz D, Grundey J, Paulus W, Nitsche M

(2010). Dosage-dependent non-linear effect of L-dopa on human motor cortex plasticity. J Physiol 588: 3415-3424.

Mori K, Togashi H, Kojima T, Matsumoto M, Ohaishi S, Ueno K et al (2001). Different effects of anxiolytic agents, diazeoam and 5-HT(1A) agonist tandospirone, on hippocampal long-term potentiation in vivo. Pharmacol Biochem Behav 69: 367-372.

Nitsche M, Grundey J, Liebetanz D, Lang N, Tergau F, Paulus W (2004). Catecholaminergic consolidation of motor cortical neuroplasticity in humans. Cereb Cortex 14: 1240-1245.

Nitsche MA, Cohen LG, Wassermann EM, Priori A, Lang N, Antal A et al (2008). Transcranial direct surrent stimulation: state of art 2008. Brain Stimul 1: 206-223.

Nitsche MA, Fricke K, Henschke U, Schlitterlau A, Liebtanz D, Lang $\mathrm{N}$ et al (2003b). Pharmacological modulation of cortical excitability shifts induced by transcrnial DC stimulation. J Physiol 533: 293-301.

Nitsche MA, Klein C, Tergau F, Rothwell J, Paulus W (2003a). Level of action of cathodal DC polarization induced inhibition of the human motor cortex. Clin Neurophysiol 144: 600-604.

Nitsche MA, Kuo M, Karrasch R, Warden B, Liebtanz D, Paulus W (2009). Serotonin affects transcrnial direct current (tDCS)induced neuroplasticity in humans. Biol Psychiatry 66: 503-508.

Nitsche MA, Paulus W (2000). Excitability changes induced in the human motor cortex by weak transcranial direct current stimulation. J Physiol 527: 633-639.

Nitsche MA, Paulus W (2001). Sustained excitability elevations induced by transcranial DC motor cortex stimulation in humans. Neurology 57: 1899-1901.

Nitsche MA, Roth A, Kuo NF, Fischer AK, Liebetanz D, Lang N (2007). Timing-dependent modulation of associative plasticity by general network excitability in the human motor cortex. J Neurosci 27: 3807-3812.

Normann C, Clark K (2005). Selective modulation of $\mathrm{Ca}(2+)$ influx pathways by 5 -HT regulates synaptic long-term plasticity in the hippocampus. Brain Res 1037: 187-193.
Normann C, Schmitz D, Furmaier A, Doing C, Bach M (2007). Long-term platsicity of visually evoked potentials in humans is altered in major depression. Biol Psychiatry 62: 373-380.

Panicker MM, Parker I, Miledi R (1991). Receptors of the serotonin $1 \mathrm{C}$ subtype expressed from cloned DNA mediate the closing of $\mathrm{K}+$ membrane channels encoded by brain mRNA. Proc Natl Acad Sci USA 88: 2560-2562.

Park S, Jang H, Cho K, Kim M, Yoon S, Rhie D (2012), Developmental switch of the serotonergic role in the induction of synaptic long-term potentiarion in the rat visual cortex. Korean J Physiol Pharmacol 16: 65-70.

Pittenger C, Duman R (2007). Stress, depression and neuroplasticity: a convergence of mechanisms. Neuropharmachology 33: 88-109.

Player MJ, Taylor JL, Weikert CS, Alonzo A, Sachdev PS, Martin D et al (2014). Increase in PAS-induced neuroplasticity after a treatment course of transcranial direct current stimulation for depression. J Affect Disord 167: 140-147.

Rajii TK, Sun Y, Zomorrodi-Moghaddam R, Farzan1 F, Blumberger DM, Benoit Mulsant BH et al (2013). PAS-induced potentiation of cortical-evoked activity in the dorsolateral prefrontal cortex. Neuropsychopharmacology 38: 2545-2552.

Reiser G, Donie F, Binmoller FJ (1989). Serotonin regulates cytosolic $\mathrm{Ca} 2+$ activity and membrane potential in a neuronal and in a glial cell line via 5-HT3 and 5-HT2 receptors by different mechanisms. J Cell Sci 93: 545-555.

Savaskan E, Muller S, Boehringer A, Schulz A, Schaechinger H (2008). Antidepressive therapy with escitalopram improves mood, cognitive sumptoms, and identity memory for angry faces in elderly depressed patients. Int J Neuropsychopharmacol 11: 381-388.

Schaechter J (2004). Motor rehabilitation and brain plasticity after hemiparetic stroke. Prog Neurobiol 73: 61-72.

Stassen HH, Angst J (2012). Delayed onset of action of antidepressant. CNS Drugs 9: 177-184.

Staubli U, Otaky N (1994). Serotonin controls the magnitude of LTP induced by theta bursts via action on NMDApreceptor-mediated responses. Brain Res 643: 10-16.

Stephan K, Kunesch E, Cohen LG, Benecke R, Classen J (2000). Induction of plasticity in the human motor cortex by paired associative stimulation. Brain 123: 572-584.

Thirugnanasambandam N, Grundey J, Paulus W, Nitsche MA (2011). Dose-dependent nonlinear effect of L-DOPA on paired associative stimulation-induced neuroplasticity in humans. J Neurosci 31: 5294-5299.

Wood MD, Wren PB (2008). Serotonin-dopamine interactions: omplications for the design of novel therapeitic agents for psychiatric disorders. Prog Brain Res 172: 213-230.

Ziemann U, Paulus W, Nitsche M, Pascual-Leone A, Byblow W, Berardelli A et al (2008). Consesus: motor cortex plasticity protocols. Brain Stimul 1: 164-182. 


\subsection{Acute and chronic effects of noradrenergic enhancement on transcranial direct current stimulation (tDCS)-induced neuroplasticity in humans}

Similar to serotonine, noradrenaline is thought to act on plasticity in the nervous system, which furthermore affects cognition and motor learning (Kemp \& Manahan-Vaughan, 2008; Marzo et al., 2009). Studies have shown that both, acute and chronic administration of the selective noradrenaline reuptake inhibitor (NRI) reboxetine (RBX) restored spatial learning deficits and hippocampal synaptic plasticity in an animal model of depression (Bhagya et al., 2015). In human studies, acute administration of RBX improves cognition and motor performance in healthy and depressed subjects (Ferguson et al., 2003; Wang et al., 2009). Thus NRI might have a potential for clinical application in various neurological and psychiatric diseases accompanied by pathological alterations of plasticity. A previous study has shown that a single dose of the monoamine reuptake inhibitor amphetamine enhances the duration of the aftereffects of anodal transcranial direct current stimulation (tDCS) (Nitsche et al., 2004). Furthermore, both the aftereffects induced by anodal and cathodal tDCS were reduced by a $\beta$-adrenergic receptor blocker (Nitsche et al., 2004). The results of this study suggest that the adrenergic system significantly impacts on plasticity in humans. In clinical applications, it usually takes several weeks to obtain therapeutic effects through selective NRI (Kasper et al., 2000). Thus it might be specifically relevant to learn about the impact of chronic noradrenergic activity enhancement on physiological processes. Interestingly, chronic administration of the selective serotonin reuptake inhibitor (SSRI) citalopram increased and prolonged tDCS-induced long term potentiation (LTP)-like plasticity in healthy subjects as compared to placebo in our previous study (Kuo et al., 2016). Since serotonin and noradrenaline are both neuromodulators and are effective for treating depression, they 
might show some similar patterns to the same plasticity-induction protocol. Nevertheless, related studies regarding chronic effects of noradrenlaine on neuroplasticity in humans are rare. The aim of the study was thus to explore the impact of single dose and chronic administration of the NRI RBX, on plasticity induced by tDCS in healthy humans via a double-blinded, placebo-controlled, randomized crossover study. 16 healthy volunteers received placebo or single dose RBX (8mg) before anodal or cathodal tDCS of the primary motor cortex. Afterwards, the same subjects took RBX ( $8 \mathrm{mg} /$ day) consecutively for 21 days. During this period, two additional interventions were performed (RBX with anodal or cathodal tDCS), to explore the impact of chronic RBX treatment on plasticity. Plasticity was monitored by motor evoked potential amplitudes elicited by transcranial magnetic stimulation. Chronic administration of RBX increased and prolonged the LTP-like plasticity induced by anodal tDCS for over 24 hours. It significantly converted cathodal tDCS-induced long term depression (LTD)-like plasticity into facilitation, as compared to the single dose condition, for 120 minutes after stimulation. The results show a prominent impact of noradrenaline receptor enhancement on plasticity of the human brain. Specifically, noradrenergic enhancement fosters the impact of brain stimulation by reinforcing facilitatory plasticity. 
Title Page

\section{Acute and chronic effects of noradrenergic enhancement on transcranial direct current stimulation (tDCS)-induced neuroplasticity in humans}

Hsiao-I Kuo ${ }^{12}$, Prof. Dr. Walter Paulus ${ }^{1}$, Dr. Giorgi Batsikadze ${ }^{14}$, Asif Jamil ${ }^{12}$, Dr. Min-Fang Kuo ${ }^{2}$, Prof. Dr. Michael A. Nitsche $e^{1,2,3 *}$

${ }^{1}$ Department of Clinical Neurophysiology, University Medical Center, Georg-August-University, Robert -Koch-Straße 40, 37075 Göttingen, Germany

${ }^{2}$ Dept. Psychology and Neurosciences,Leibniz Research Centre for Working Environment and Human Factors, Ardeystrasse 67, Dortmund, Germany

${ }^{3}$ Department of Neurology, University Medical Hospital Bergmannsheil, Bochum, Germany ${ }^{4}$ Department of Neurology, Essen University Hospital, University of Duisburg-Essen, Germany

*Corresponding author: Prof. Dr. M.A. Nitsche, Department Psychology and Neuroscience , Leibniz Research Centre for Working Environment and Human Factors, Ardeystrasse 67, Dortmund Germany. Tel: +49 2311084 301, Fax: +49 55139 8126. E-mail: nitsche@ifado.de

Running title: long term impact of noradrenaline on neuroplasticity

Key words: noradrenaline, neuroplasticity, transcranial direct current stimulation 


\section{Key point summary}

1. Chronic administration of the selective noradrenaline reuptake inhibitor (NRI) reboxetine (RBX) increased and prolonged the long term potentiation (LTP)-like plasticity induced by anodal transcranial direct current stimulation (tDCS) for over 24 hours.

2. Chronic administration of RBX converted cathodal tDCS-induced long term depression (LTD)-like plasticity into facilitation for $120 \mathrm{~min}$.

3. Chronic noradrenergic activity enhancement on plasticity of the human brain might partially explain the delayed therapeutic impact of selective NRIs in depression and other neuropsychiatric diseases. 


\begin{abstract}
Noradrenaline affects cognition and motor learning processes via its impact on long-term potentiation (LTP) and depression (LTD). We aimed to explore the impact of single dose and chronic administration of the selective noradrenaline reuptake inhibitor (NRI) reboxetine (RBX) on plasticity induced by transcranial direct current stimulation (tDCS) in healthy humans via a double-blinded, placebo-controlled, randomized crossover study. 16 healthy volunteers received placebo or single dose RBX (8mg) before anodal or cathodal tDCS of the primary motor cortex. Afterwards, the same subjects took RBX (8 mg/day) consecutively for 21 days. During this period, two additional interventions were performed (RBX with anodal or cathodal tDCS), to explore the impact of chronic RBX treatment on plasticity. Plasticity was monitored by motor evoked potential amplitudes elicited by transcranial magnetic stimulation. Chronic administration of RBX increased and prolonged the LTP-like plasticity induced by anodal tDCS for over 24 hours. Chronic RBX significantly converted cathodal tDCS-induced LTD-like plasticity into facilitation, as compared to the single dose condition, for 120 minutes after stimulation. The results show a prominent impact of chronic noradrenergic enhancement on plasticity of the human brain which might partially explain the delayed therapeutic impact of selective NRIs in depression and other neuropsychiatric diseases.
\end{abstract}

Abbreviations list: LTP, long-term potentiation; LTD, long-term depression; NRI, noradrenaline reuptake inhibitor; PLC, placebo; RBX, reboxetine; tDCS, transcranial direct current stimulation 


\section{Introduction}

Noradrenaline affects learning and memory processes via modulating long-term potentiation (LTP) and depression (LTD) (Tully et al., 2007; Wallings et al., 2016). The precise effect of noradrenaline on plasticity, as explored in animal models, is complex and depends on receptor subtype, concentration, and the site of action (Marzo et al., 2009). Specifically, noradrenaline affects the direction of LTP as well as LTD. Its specific impact depends on the activation of $\alpha$ - and $\beta$-adrenoreceptors (Kemp \& Manahan-Vaughan, 2008; Marzo et al., 2009). Activation of adrenoreceptors affects various intracellular factors and modifications of ion channel opening (Marzo et al., 2009). Importantly, adrenoreceptors also impact on N-methyl-D-aspartate (NMDA) and gamma-aminobutyric acid (GABA) receptors, and therefore influence the direction of LTP and LTD (Lei et al., 2007).

Noninvasive brain stimulation techniques such as transcranial direct current stimulation (tDCS) have been established to induce LTP- and LTD-like cortical excitability alterations in humans (Nitsche \& Paulus, 2000). TDCS induces polarity-dependent plasticity via its primary subthreshold effects on resting membrane potentials (Purpura \& McMurtry, 1965; Nitsche et al., 2007). Anodal stimulation elicits neural depolarization which enhances cortical excitability, whereas cathodal tDCS results in neural hyperpolarization which diminishes cortical excitability. Stimulation for some minutes results in respective neuroplastic effects, which depend on glutamatergic mechanisms and are calcium-dependent (Nitsche \& Paulus, 2000, 2001; Nitsche et al., 2003a; Nitsche at al., 2005). Nevertheless, tDCS-induced plasticity differs from classical plasticity induction protocols, used primarily in vitro. 
The latter involve not tonic subthreshold, but pulsatile suprathreshold stimulation. Thus, mechanisms of plasticity might not be identical, although similarities like NMDA receptor dependency, and calcium dependency, are present (Liebtanz et al., 2002; Nitsche et al., 2003b). A previous study has shown that acute administration of the monoamine reuptake inhibitor amphetamine enhances the duration of the aftereffects of anodal tDCS (Nitsche et al., 2004). Furthermore both, the aftereffects induced by anodal and cathodal tDCS, were reduced by a $\beta$-adrenergic receptor blocker (Nitsche et al., 2004). The results of this study suggest that the adrenergic system significantly impacts on plasticity in humans, which is in accordance with the results from animal studies (Nakadate et al., 2006; Marzo et al., 2009).

Psychiatric diseases such as major depression are accompanied by compromised LTP, which can be restored by antidepressant treatment (Campell \& Macqueen, 2004; Castren, 2004). Patients with major depression show reduced LTP-like plasticity, as compared with healthy controls, and administration of antidepressant agents can increase LTP-like plasticity (Normann et al., 2007). Furthermore, chronic administration of the selective noradrenaline reuptake inhibitor (NRI), reboxetine (RBX), restored spatial learning deficits and hippocampal synaptic plasticity in an animal model of depression (Bhagya et al., 2015). For studies in humans, acute administration of RBX improves cognition and motor performance in healthy and depressed subjects (Ferguson et al., 2003; Plewnia et al., 2004; Wang et al., 2009). In summary, selective NRIs might partially exert their treatment effects by enhancing LTP-like plasticity in depression, and therefore improve learning and cognition. Thus selective NRIs might have a potential for treatment of psychiatric diseases accompanied by pathological alterations of plasticity. Knowledge about the impact of 
noradrenergic enhancement in humans on plasticity is however limited at present. Clinically, it usually takes weeks to obtain therapeutic effects through selective NRIs (Kasper et al., 2000), such it might be relevant to learn about the impact of chronic noradrenergic activity enhancement on physiological processes.

Here, we explored the impact of single dose and chronic noradrenergic receptor activity enhancement via administration of the selective NRI RBX, on tDCS-induced motor cortical plasticity. We hypothesized that RBX increases LTP-like plasticity induced by anodal tDCS, whereas cathodal tDCS-induced LTD-like plasticity should be abolished or converted into excitation. Additionally, a foregoing study found that chronic administration of the selective serotonin reuptake inhibitor (SSRI) citalopram increased and prolonged tDCS-induced LTP-like plasticity in healthy subjects as compared to placebo (Kuo et al., 2016). This might indicate more stable serotonergic enhancement or upregulation of respective receptors due to chronic administration (Pariente et al., 2001; Coppell et al., 2003). Since serotonin and noradrenaline are both neuromodulators and are effective for treating depression, they might show some similar patterns to the same plasticity-induction protocol. We furthermore hypothesized that chronic administration of RBX should induce more prominent effects as compared to single dose administration.

\section{Material and Methods}

Ethical approval

The study was approved by the ethics committee of the University Medical Center of Goettingen, and we conformed to the standards set by the Declaration of Helsinki 
(2008 version). Written informed consent was obtained from all subjects who participated in the study before inclusion.

Subjects

Sixteen right-handed, healthy subjects participated in the experiments ( 8 males, age 27.5 \pm 4.01 (standard deviation) years). Subjects were all right-handed, and between 18 and 50 years old. They had no history of chronic or acute neurological, psychiatric, or medical diseases, no family history of epilepsy, no present pregnancy, no cardiac pacemaker, no previous surgery involving implants to the head (cochlear implants, aneurysm clips, brain electrodes), and absent acute or chronic medication or drug intake, including nicotine consumption. Participants familiar with non-invasive brain stimulation and pharmacological studies were preferred. However, responder or non-responder status (i.e., to tDCS) did not serve as criterion to include or exclude participants and we did not check for genetic polymorphisms (brain-derived neurotrophic factor (BDNF), catechol-O-methyl transferase (COMT), or others).

Transcranial direct current stimulation

Direct current was applied through a pair of saline-soaked surface sponge electrodes $\left(35 \mathrm{~m}^{2}\right)$ and delivered by a battery-driven constant current stimulator (neuroConn $\mathrm{GmbH}$, Ilmenau, Germany) with a maximum output of $4.5 \mathrm{~mA}$. The stimulating electrode was placed over the representational hotspot of the right abductor digiti minimi muscle (ADM) identified with transcranial magnetic stimulation (TMS), and the return electrode was placed contralaterally above the right orbit. A current strength of $1 \mathrm{~mA}$ was administered for 9 min for cathodal tDCS and 13 min for anodal tDCS. These stimulation durations induce cortical excitability alterations lasting 
approximately for 1 hour after the end of stimulation (Nitsche \& Paulus, 2001; Nitsche et al., 2003a). In our study, we aimed to keep the classical protocol identical to other pharmacology-tDCS studies in our lab to enhance comparability between studies (Kuoet al., 2008; Monte-Silva et al., 2010; Fresnoza et al., 2014 ).

Pharmacological interventions

Reboxetine (RBX) (8mg) (Winthrop Arzneimittel GmbH, Frankfurt am Main, Germany) or equivalent placebo medication (PLC) (Pfizer Italia S.r.l., Ascoli Piceno, Italy) was administered 2 hours before the start of the experimental sessions. A sufficient plasma level of RBX is achieved 2 hours after oral intake, and the respective dose is sufficient to elicit prominent effects in the central nervous system (Dostert et al., 1997). Steady state plasma concentrations are achieved after five days of drug intake (Pellizzoni et al., 1996). In clinical application, the majority of antidepressants have therapeutic effects after approximately two weeks of treatment (Dostert et al., 1997; Kasper et al., 2000). Therefore, we choose three weeks administration of RBX for the chronic part of experiments, and started plasticity induction procedures after 2 weeks of administration in the chronic medication condition.

\section{Monitoring of motor cortical excitability}

TMS-elicited motor evoked potentials (MEPs) were recorded to monitor excitability changes of the motor cortical representation of the right abductor digiti minimi muscle (ADM). Single-pulse TMS was conducted by a Magstim 200 magnetic stimulator (Magstim, Whiteland, Dyfed, UK) with a figure-of-eight magnetic coil (diameter of one winding=70 $\mathrm{mm}$, peak magnetic field=2.2 $\mathrm{T}$ ). The coil was held 
tangentially to the skull, with the handle pointing backwards and laterally at an angle of $45 \mathrm{deg}$ from midline, inducing a posterior-anterior current flow direction in the motor cortex. The optimal coil position (hotspot) was defined as the site where stimulation consistently resulted in the largest MEPs of the contralateral ADM. Surface EMG was recorded from the right $\mathrm{ADM}$ with $\mathrm{Ag}-\mathrm{AgCl}$ electrodes in a belly-tendon montage. The signals were filtered ( $2 \mathrm{~Hz}$ to $2 \mathrm{kHz}$, sampling rate $5 \mathrm{kHz}$ ), amplified, and then stored on computer via a power 1401 data acquisition interface (Cambridge Electronic Design, Cambridge, UK).

\section{Experimental procedures}

The study was conducted in a double-blinded, counter-balanced and placebo-controlled design. Each subject participated in six experimental sessions, including single dose RBX (the first 4 sessions) and chronic RBX (the last 2 sessions), or the respective placebo medication. For the single dose part, subjects received PLC or RBX combined with anodal or cathodal tDCS. Each experimental session was separated by one week to avoid cumulative effects. In the chronic RBX part, participants received RBX (8mg/day) consecutively for 21 days. The two sessions under chronic RBX application (RBX with anodal/cathodal tDCS) were conducted in counterbalanced order at the end of the second and third weeks after the start of chronic drug intake. At the beginning of each session, subjects were seated in a comfortable chair with head and arm rest. First the hotspot of the right ADM was determined over the left primary motor cortex, and 20 MEPs were recorded with the TMS intensity eliciting averaged $1 \mathrm{mV}$ MEP as the first baseline. Two hours after administration of the medication, a second baseline was obtained with the same intensity as BL2 to assess a possible influence of the medication on cortical 
excitability, and a third baseline was recorded if necessary (if BL2 MEP was outside the range of 80 to $120 \%$ of BL1 MEP) with adjusted TMS intensity for $\sim 1 \mathrm{mV}$ MEP amplitude. Afterwards, tDCS was performed between 12 am and $1 \mathrm{pm}$. Immediately after tDCS, 20 MEPs were recorded at the time points of $0,5,10,15,20,25,30,60$, 90, and $120 \mathrm{~min}$, and also the same evening (se: between $6 \mathrm{pm}$ and $7 \mathrm{pm}$ ), next morning (nm: between 9 am and $10 \mathrm{am}$ ), next noon (nn: between 12 am and $1 \mathrm{pm}$ ), and next evening (ne: between $6 \mathrm{pm}$ and $7 \mathrm{pm}$ ) (Figure 1).

\section{Statistics}

The individual MEP amplitude means of baseline 1,2,3, and all time points after stimulation were calculated. After checking for normal distribution (Shapiro-Wilk test), a repeated-measure analysis of variance (ANOVA) for the time bins up to next evening after stimulation was calculated with the within subject factors time course, drug condition (placebo medication, single dose RBX and chronic RBX), stimulation type (anodal and cathodal) and the dependent variable raw MEP amplitude, including baseline 2, or, in case of adjustment of MEP amplitudes, baseline 3. The Mauchly test of sphericity was conducted, and the Greenhouse-Geisser correction was applied when necessary. In case of significant results of the ANOVA, post hoc comparisons were performed using Student's t tests (paired samples, two tailed, critical $p<0.05$, not corrected for multiple comparisons) to determine whether the MEP amplitudes before and after tDCS differed in each intervention condition and whether these differences depended on drug condition. Furthermore, we conducted an ANCOVA with baseline raw MEP (baseline 2, or, in case of adjustment of MEP amplitudes, baseline 3) as co-variate, and for the chronic medication condition with order as co-variate to rule out systematic effects of order of conditions on the results. 
To explore if baseline MEP, and TMS intensity (percentage of maximal stimulator output, \%MSO) needed to obtain baseline MEP differed between each session, the respective baselines were compared via Student's t-tests (paired samples, two tailed, $\mathrm{p}<0.05$, not corrected for multiple comparisons).

\section{Results}

All subjects tolerated tDCS and RBX well. None of them reported any side effects of either RBX or the stimulation upon request. RBX alone did not change baseline MEP amplitudes and stimulation intensity significantly, as revealed by Student's t-tests comparing MEP between baseline 1 and baseline 2 (paired samples, two-tailed, $\mathrm{p}>0.05)$ (Table 1).

The Shapiro-Wilk test indicted that all data were normally distributed (all $\mathrm{p}>0.05$ ). The results of the ANOVA showed significant main effects of drug $(F(2)=7.843$; $\mathrm{p}=0.006)$, stimulation $(\mathrm{F}(1)=19.852 ; \mathrm{p}=0.002)$, and significant drug $\mathrm{x}$ stimulation $(\mathrm{F}(2)=10.159 ; \mathrm{p}=0.007)$, and stimulation $\mathrm{x}$ time $(\mathrm{F}(14)=4.964 ; \mathrm{p}=0.005)$ interactions. In addition, the result of ANCOVA did not show a significant effect of baseline MEP (baseline 2, or, in case of adjustment of MEP amplitudes, baseline 3) ( $\mathrm{P}=0.723)$, and the chronic medication conditions did not result in a significant effect of order $(\mathrm{p}=$ 0.617) (Table 2).

As revealed by the respective post hoc test, under placebo medication, cathodal tDCS decreased MEP amplitudes for up to 60 min after stimulation, whereas anodal tDCS 
increased cortical excitability for $90 \mathrm{~min}$. Single dose administration of RBX enhanced the MEP amplitudes significantly as compared to placebo medication at the time points of 10, 15, and 25 min after anodal tDCS, whereas it reversed cathodal tDCS-induced inhibition into facilitation, which remained significant for up to $60 \mathrm{~min}$ after tDCS. Under chronic RBX, anodal tDCS resulted in larger MEP amplitudes compared to placebo medication at the time point of $10,15,20,25,30,60,120$, se, $\mathrm{nm}$, nn, and ne after tDCS application. Furthermore, compared to the single dose condition, chronic RBX significantly increased MEP amplitudes at the time points of 20, 25, 30, 60, 120, se, nm, nn, and ne, which means the MEP amplitude enhancement was present for more than 24 hours after tDCS until next evening after stimulation. For cathodal tDCS, chronic RBX showed a similar effect as single dose administration compared to the placebo condition, which converted inhibition into facilitation with a more prominent excitability enhancement for 120 minutes after stimulation. In addition, chronic RBX resulted in a significant enhancement of MEP amplitudes compared to the single dose condition for 30 minutes and at the time point of 90 minutes after cathodal tDCS (Figure 2).

\section{Discussion}

The results of this study show that single dose administration of the selective NRI RBX increased LTP-like plasticity induced by anodal tDCS, whereas it turned cathodal tDCS-induced LTD-like plasticity into facilitation in healthy subjects. Moreover, under chronic administration, the LTP-like effects of anodal tDCS were prolonged for more than 24 hours after intervention, and thus lasted relatively longer than those under single dose administration or placebo medication. For cathodal 
tDCS, chronic RBX showed larger LTP-like plasticity compared to single dose medication for 120 minutes after stimulation. The prolonged after-effects of anodal tDCS achieved by chronic RBX might explain the relevance of long-term administration of selective NRIs to exert optimal effects. Moreover, these findings support recent concepts that changes of neural plasticity are relevant for therapeutic effects of noradrenergic enhancement (Marzo et al., 2009; Bhagya et al., 2015).

Baseline MEP showed no significant differences after RBX intake, which differs from another study (Plewnia et al., 2002; Plewnia et al., 2004). This difference between study results might be due to the fact that we used a stimulation intensity which elicits single pulse MEPs with peak-to-peak amplitudes on average $1 \mathrm{mV}$ instead of $180 \%$ MT (motor threshold) in the foregoing one. The latter criterion will result in larger MEPs. The respective different effect of RBX on baseline MEP might be caused by different pharmacological mechanisms involved in low and high intensity parts of the recruitment curve. Thus high, but not low amplitude MEPs are relevantly affected by the glutamatergic system (Paulus et al., 2008).

\section{Mechanisms}

Our results are in accordance with the findings of a previous experiment, in which the monoamine reuptake inhibitor amphetamine prolonged the duration of the LTP-like after-effects induced by anodal tDCS (Nitsche et al., 2004). They are also in line with results of animal slice experiments. Noradrenergic enhancement can enhance LTP and block LTD (Katsuki et al., 1997; Tully et al., 2007). The specific mechanism responsible for the effects of $\mathrm{RBX}$ on motor cortex plasticity in the human brain remains to be clarified in future studies. One candidate mechanism is the decrease of 
potassium conductance by the drug (Marzo et al., 2009). This would result in a depolarization of postsynaptic membranes and enhance calcium influx into the intraneuronal compartment through NMDA receptors and voltage-dependent calcium channels $(\mathrm{Gu}, 2002)$. The direction of induced plasticity depends on the amount of intracellular calcium. High enhancement of intracellular calcium induces LTP, whereas low enhancement results in LTD (Lisman, 2001). Since the after-effects of tDCS are NMDA receptor- and calcium-dependent (Liebtanz et al., 2002; Nitsche et al., 2003b), RBX might have strengthened the excitability enhancement induced by anodal tDCS through an enhancement of calcium influx, which might prolong the after effects of anodal tDCS. For cathodal tDCS, RBX might have shifted a small to large calcium increase through this mechanism and thus converted inhibition into facilitation. The calcium influx might be still lower for cathodal than for anodal tDCS, which would explain that the after effects of cathodal tDCS were shorter lasting. However, this suggested mechanism is speculative at present. Which specific noradrenaline receptor subtypes are involved in this mechanism is not clear. $\beta$-adrenoreceptors might be candidates, since in vivo and vitro studies conducted in the dentate gyrus and in area CA1 of the hippocampus show that noradrenaline facilitates or induces LTP through $\beta$-adrenoreceptors (Katsuki et al., 1997). Furthermore, since $\beta$-adrenoreceptors decrease calcium activation-dependent potassium conductance (Hass \& Konnerth, 1983), they are relevant for the conversion from early to late LTP (Straube \& Frey, 2003). Because noradrenaline also affects acetylcholine, serotonin, dopamine release and GABAergic activation (Page \& Lucki, 2002), which have been shown to modulate tDCS-induced plasticity, it cannot be ruled out that these modulators might have some impact on the effects (Kuo et al., 2007; Kuo et al., 2008; Nitsche et al., 2009). Although the results of the present study 
are rather clear, it should be kept in mind that in this study we explored a specific dosage of RBX, which is not selective for a specific noradrenergic receptor, and explored plasticity of a specific cortical area. The effects of activation of adrenergic receptor subtypes on LTP and LTD might however differ (Katsuki et al., 1997; Kemp \& Manahan-Vaughan, 2008). Moreover, due to the neuromodulatory function of noradrenaline, noradrenergic activation might exert non-linear dosage-dependent effects, and specific effects might depend on receptor density of a specific area, which differ between regions (Katsuki et al., 1997; Marzo et al., 2009). These factors might explain at least partially conflicting results between studies. Future studies should thus consider the contribution of specific receptor subtypes, explore the impact of different dosages, and explore plasticity effects of noradrenaline in different cortical areas. These factors have been shown to contribute to the effects of other neuromodulators, such as dopamine, on plasticity (Kuo et al., 2008; Monte-Silva et al., 2010).

Functional implications

Previous studies have shown that acute administration of RBX improves cognitive and motor performance in healthy subjects and depressed patients (Ferguson et al., 2003; Wang et al., 2009). Clinically, the majority of selective NRIs currently available has therapeutic effects only after approximately 2 weeks of treatment (Kasper et al., 2000), suggesting that in addition to the rapid inhibition of noradrenaline reuptake, other long-term adaptive modifications are induced by chronic noradrenergic enhancement. Here, its impact on LTP-like plasticity is a candidate mechanism. Indeed, deficient LTP was restituted in patients suffering from major depression after successful therapy (Player et al., 2014). In our study, repeated treatment with RBX 
significantly enhanced and prolonged LTP-like plasticity induced by anodal tDCS for more than $24 \mathrm{~h}$, suggesting induction of late phase LTP, whereas for single dose administration the after-effects of anodal tDCS were only extended until the evening of the day of intervention. In accordance, animal models of depression suggest that repeated treatment with selective NRIs significantly restores hippocampal synaptic plasticity and reduces spatial learning deficits (Marzo et al., 2009; Bhagya et al., 2015). These findings indicate the possibility that the enhancement of synaptic plasticity may contribute to adaptive changes induced by long-term antidepressant treatment. Interestingly, we found similar effects for the SSRI citalopram (Kuo et al., 2016). If this is a relevant mechanism, which can explain the delayed effects of antidepressants on clinical symptoms, remains to be shown directly. Beyond depression, compromised plasticity plays a role in various neurological and psychiatric diseases, and restitution by interventional approaches might be an important mechanism for reducing clinical symptoms. Noradrenergic enhancement might be relevant for diseases which are accompanied by deficient LTP, such as post stroke rehabilitation, and Parkinson's disease, just to name a few. It was described that RBX can improve hand function in chronic stroke patients (Zittel et al., 2007). Moreover, tDCS has been introduced as a potential therapeutic tool for diverse neurological and psychiatric diseases (Flöel, 2014; Kuo et al., 2014). Given the strengthening effect of RBX on the aftereffects of tDCS, combining tDCS with selective NRIs might be a promising option to enhance the clinical impact of these interventions. Indeed, such synergistic effects have been demonstrated for the combination of a SSRI and tDCS as antidepressant therapy (Brunoni et al., 2013).

\section{Limitations}


Some potential limitations of the present study should be considered. First, due to the limited time frame, we explored only three weeks for chronic administration of RBX. Second, to rule out an interference effects of plasticity interventions, a one week intersession interval was required. Since we did not have the chance to explore behavioral effects of noradrenaline in the present study, thus presumed functional implications are speculative at present. Third, we did not obtain drug plasma levels, which would have enabled exploration of dosage-dependent effects of the medication to some extent. Moreover, participants had not the exact identical duration of RBX intake in the respective chronic medication conditions (medication duration between 14 and 21 days). Participants received anodal stimulation after two weeks and cathodal stimulation after three weeks of RBX, or vice versa. To rule out systematic effects of order of conditions on the results, we conducted an ANCOVA for the chronic medication condition with order as co-variate, which ruled out an order effect.

Future studies

Since animal experiments showed different effects of $\alpha$ and $\beta$ receptors on plasticity (Kemp \& Manahan-Vaughan, 2008; McElligott \& Winder, 2008; Wojtowicz et al., 2010), future studies should consider the contribution of specific receptor subtypes (i.e., $\alpha_{1}, \alpha_{2}$ and $\beta_{1}, \beta_{2}, \beta_{3}$ subtypes) in more detail. Moreover, we have found a "focusing effect" of another neuromodulator, namely dopamine, on neuroplasticity, which strengthened focally induced but weakened/reversed nonfocally induced plasticity (Kuo et al., 2008; Fresnoza et al., 2014 ). In contrast, RBX might induce a de-focusing effect on plasticity, which would be an interesting difference to other neuromodulators. Therefore, future studies should consider to explore the noradrenergic effects with different plasticity induction protocols such as paired 
associative stimulation (PAS), which induce more focally restricted plasticity. Given the variability of neuromodulatory brain stimulation interventions (López-Alonso et al., 2014; Wiethoff et al., 2014), replication of the results of this study is also warranted. Finally, the results of the study were obtained in healthy volunteers. In neuropsychiatric diseases, transmitter availability and other features of brain function might be different. Thus, the assessment of the current setup in patients groups suffering from depression, stroke and other neurological and psychiatric syndromes in which the adrenergic system or neuroplasticity are involved is required to explore transferability of results, and mechanisms.

Our results show modulatory effects of the selective NRI, RBX, on tDCS-induced plasticity in the human motor cortex. RBX shifted tDCS-induced plasticity into a facilitatory direction. This impact of noradrenaline on plasticity may be a relevant neurological foundation for the therapeutic effect of selective NRIs in depressed subjects. This finding also suggests that long-term administration of selective NRIs might be a promising pathway to treat patients with neurological deficits associated with compromised LTP-like plasticity. Furthermore, the results may help to understand neuroplasticity processes in the human brain on the rational basis of pharmacological intervention. 


\section{References}

Bhagya V, Srikumar B, Traju T \& Rao B. (2015). The selective noradrenergic reuptake inhibitor reboxetine restores spatial learning deficits, biochemical changes, and hippocampal synaptic plasticity in an animal model of depression. Jounal of Neuroscience Research93, 104-120.

Brunoni A, Valiengo L, Baccaro A, Zanao T, Oliveira JD \& Goulart A. (2013). The sertraline vs electrical current therapy for treating depression clinical study: results from a factorial, randomized, controlled trial. JAMA Psychiatry70, 1-9.

Campell S \& Macqueen G. (2004). The role of the hippocampus in the pathophysiology of major depression. J Psychiatry Neurosci29, 417-426.

Castren E. (2004). Neurotrophic effects of antidepressant drugs. Curr Opin Pharmacol4, 58-64.

Coppell A, Pei Q \& Zetterstrom T. (2003). Bi-phasic change in BDNF gene expression following antidepressant drug treatment. Neuropharmacology44, 903-910.

Dostert P, Benedetti M \& Poggesi I. (1997). Review of the pharmacokinetics and metabolism of reboxetine, a selective noradrenaline reuptake inhibitor. European Neuropsychopharmacology1, 23-35.

Ferguson J, Wesnes K \& Schwartz G. (2003). Reboxetine versus paroxetine versus placebo: effects on conitive functioning in depressed patients. International Clinical Psychopharmacology18, 9-14.

Flöel A. (2014). tDCS-enhanced motor and cognitive function in neurological diseases. Neuroimage85, 934-947.

Fresnoza S, Stiksrud E, F Klinker, D Liebetanz, W Paulus, M F Kuo \& Nitsche. MA. (2014 ). Dosage-dependent effect of dopamine D2 receptor activation on motor cortex plasticity in humans. J Neurosci34, 10701-10709.

Gu Q. (2002). Neuromodulatory transmitter systems in the cortex and their role cortical plasticity. Neuroscience111, 815-835.

Hass H \& Konnerth A. (1983). Histamine and noradrenaline decrease calcium-activated potasium conductance in hippocampal pyramidal cells. Nature302, 432-434.

Kasper S, Giamal N \& Hilger E. (2000). Reboxetine: the first selective noradrenaline re-uptake inhibitor. Exp Opin Pharmacother1, 771-782.

Katsuki H, Izumi Y \& Zorumski C. (1997). Nordrenergic regulation of synaptic plasticity in the hippocampal CA1 region. Jounal of Neurophysiology77, 3013-3020. 
Kemp A \& Manahan-Vaughan D. (2008). Beta-adrenoreceptors comprise a critical element in learning-facilitated long-term platicity. Cerebral Cortex 18, 1326-1334.

Kuo HI, Paulus W, Batsikadze G, Jamil A, Kuo M \& Nitsche MA. (2016). Chronic enhancement of serotonin facilitatory excitatory transcrnial direct current-induced plasticity. Neuropsychopharmachology41, 1223-1230.

Kuo MF, Grosch J, Fregni F, Paulus W \& Nitshce MA. (2007). Focusing effect of acetylcholine on neuroplasticity in the human motor cortex. J Neurosci27, 1442-1447.

Kuo MF, Paulus W \& Nitsche MA. (2008). Boosting focally-induced brain plasticity by dopamine. Cereb Cortex18, 648-651.

Kuo MF, Paulus W \& Nitsche MA. (2014). Therapeutic effects of non-invasive brain stimulation with direct currents (tDCS) in neuropsychiatric diseases. Neuroimage15, 948-960.

López-Alonso V, Cheeran B, Río-Rodríguez D \& Fernandenz-Del-Olmo M. (2014). Inter-individual variability in response to non-invasive brain stimulation paradigms. . Brain Stimul7, 327-380.

Lei S, Deng P, Porter J \& Shin H. (2007). Adrenergic facilitation of GABAergic transmission in rat entorhinal cortex. J Neurophysiol98, 1868-1877.

Liebtanz D, Nitsche M, Tergau F \& Paulus W. (2002). Pharmacological approach to synaptic and membrane mechanisms of DC-induced neuroplasticity in man. Brain 125, 2238-2247.

Lisman J. (2001). Three Ca2+ levels affect plasticity differently: the LTP zone, the LTD zone and no man's land. J Physiol532, 285.

Marzo A, Bai J \& Otani S. (2009). Neuroplasticity regulation by noradrenaline in mammalian brain. Current Neuropharmacology7, 286-295.

McElligott Z \& Winder D. (2008). Alpha1-adrenergic receptor-induced heterosynaptic long-term depression in the bed nucleus of the stria terminalis is disrupted in mouse models of affective disorders. Neuropsychopharmachology33, 2313-2323.

Monte-Silva K, Liebetanz D, Grundey J, Paulus W \& Nitsche MA. (2010). Dosage-dependent non-linear effect of L-dopa on human motor cortex plasticity. J Physiol588, 3415-3424.

Nakadate K, Matsukawa M \& Okado N. (2006). Identification of adrenoreceptor subtype-medisted changes in the density of synapses in the rat visual cortex Neuroscience 138, 37-46.

Nitsche MA, Grundey J, Liebetanz D, Lang N, Tergau F \& Paulus W. (2004). 
Catecholaminergic consolidation of motor cortical neuroplasticity in humans. Cereb Cortex 14, 1240-1245.

Nitsche MA, Klein C, Tergau F, Rothwell J \& Paulus W. (2003a). Level of action of cathodal DC polarization induced inhibition of the human motor cortex. Clin Neurophysiol144, 600-604.

Nitsche MA, Fricke K, Henschke U, Schlitterlau A, Liebtanz D, Lang N \& et al. (2003b). Pharmacological modulation of cortical excitability shifts induced by transcrnial DC stimulation. J Physiol533, 293-301.

Nitsche MA, Seeber A, Frommann K, Klein CC, Rochford C, Nitsche MS \& et al. (2005). Modulating parameters of excitability during and after transcranial direct current stimulation of the human motor cortex. J Phsyiol568,291-303.

Nitsche MA, Kuo M, Karrasch R, Warden B, Liebtanz D \& Paulus W. (2009). Serotonin affects transcrnial direct current (tDCS)-induced neuroplasticity in humans. Biol Psychiatry66, 503-508.

Nitsche MA \& Paulus W. (2000). Excitability changes induced in the human motor cortex by weak transcranial direct current stimulation. $J$ Physiol527, 633-639.

Nitsche MA \& Paulus W. (2001). Sustained excitability elevations induced by transcranial DC motor cortex stimulation in humans. Neurology57, 1899-1901.

Nitsche MA, Roth A, Kuo NF, Fischer AK, Liebetanz D \& Lang N. (2007). Timing-dependent modulation of associative plasticity by general network excitability in the human motor cortex. J Neurosci27, 3807-3812.

Normann C, Schmitz D, Furmaier A, Doing C \& Bach M. (2007). Long-term platsicity of visually evoked potentials in humans is altered in major depression. Biol Psychiatry62, 373-380.

Page M \& Lucki I. (2002). Effects of acute and chronic reboxetine treatment on stress-induced manoamine efflux in the rat frontal cortex. Neuropsychopharmachology27, 238-247.

Paulus W, Classen J, Cohen LG, Large C, Lazzaro VD, Nitsche MA \& et al. (2008). State of the art: Pharmacologic effects on cortical excitability measures tested by transcranial magnetic stimulation. Brain Stimul 1, 151-163.

Pariente J, Loubinoux I, Carel C, Albucher J, Leger A, Manelfe C \& et al. (2001). Flouxetine modulates motor performance and cerebral activation of patients recovering from stroke. Ann Neurol50, 718-729.

Pellizzoni C, Poggesi I, Jørgensen N, Edwards D, Paus E \& Benedetti M. (1996). Pharmacokinetics of reboxetine in healthy volunteers. Single against repeated oral doses and lack of enzymatic alterations. Biopharm Drug Dispos 17, 623-633. 
Player M, Taylor J, Weickert C, Sachder P, Martin D, Mitchell P \& et al. (2014). Increase in PAS-induced neuroplasticity after a treatment course of transcranial direct current stimulation for depression. J Affect Disord160, 140-147.

Plewnia C, Hoppe J, Cohen L \& Gerloff C. (2004). Improved motor skill acquisition after selective stimulation of central norepinephrine. Neurology62, 2124-2126.

Plewnia C, Hoppe J, Heimke C, Bartles M, Cohen L \& Gerloff C. (2002). Enhancement of human cortico-motoneuronal excitability by the selective norepinephrine reuptake inhibitor reboxetine. Neuroscience letters330, 231-234.

Purpura DP \& McMurtry JG. (1965). Intracellular activities and evoked potential changes during polarization of motor cortex. J Neurophysiol28, 166-185.

Straube T \& Frey J. (2003). Involvement of beta-adrenergic receptors in protein synthesis-dependent late long-term potentiation (LTP) in the dentate gyrus of freely moving rats: the critical role of the LTP induction strength. Neuroscience119, 473-479.

Tully K, Y L, Tsvetkov E \& Bolshakov V. (2007). Norepinephrine enables the induction of associative long-term potentiation at thalamo-amygdala synapses. Pro Natl Acd Sci USA104, 14146-14150.

Wallings S, Milway J, Ingram M, Lau C, Morrison G \& Martin G. (2016). The effects of prolonged administration of norepinephrine reuptake inhibitors on long-term potentiation in dentate gyrus, and on tests of spatial and object recognition memory in rats. Neurobiol Learn Mem128, 92-102.

Wang L, Fink G, Dafotakis M \& Grefkes C. (2009). Noradrenergic stimulation and motor performance: different effects of reboxetine on movement kinetics and visuiomotor abilities in healthy human subjects. Neuropsychologia47, 1302-1312.

Wiethoff S, Hamada M \& Rothwell J. (2014). Variability in response to transcranial direct current stimulation of the motor cortex. Brain Stimul 7, 465-475.

Wojtowicz A, Fidzinski P, Heinemann U \& Behr J. (2010). Beta-adrenergic receptor activation induces long-lasting potentiation in burst-spiking but not regular-spiking cells at CA1-aubiculum synapses. Neuroscience171, 367-372.

Zittel S, Weiller C \& Liepert J. (2007). Reboxetine improves motor fucntion in chronic stroke. A pilot study. J Neurol254, 197-201. 
Table 1. MEP amplitude and stimulation intensity before and after reboxetine (RBX) administration.

\begin{tabular}{|c|c|c|c|c|c|c|}
\hline Stimulation & $\begin{array}{c}\text { TMS } \\
\text { parameter }\end{array}$ & Drug Condition & Baseline 1 & Baseline 2 & Baseline 3 & $p$ value \\
\hline \multirow[t]{6}{*}{ Anodal } & $\operatorname{MEP}(\mathrm{mV})$ & PLC & $1.04 \pm 0.12$ & $0.98 \pm 0.09$ & $0.99 \pm 0.06$ & 0.396 \\
\hline & & Acute RBX & $1.01 \pm 0.03$ & $0.95 \pm 0.18$ & $1.03 \pm 0.16$ & 0.153 \\
\hline & & Chronic RBX & $1.04 \pm 0.11$ & $1.07 \pm 0.08$ & $1 \pm 0.07$ & 0.357 \\
\hline & $\% \mathrm{MSO}$ & PLC & $52 \pm 8.07$ & $52 \pm 8.07$ & $50.3 \pm 6.74$ & 0.178 \\
\hline & & Acute RBX & $52.9 \pm 7.11$ & $52.9 \pm 7.11$ & $53.1 \pm 7.56$ & 0.445 \\
\hline & & Chronic RBX & $52.1 \pm 7.18$ & $52.1 \pm 7.18$ & $51.4 \pm 8.5$ & 0.325 \\
\hline \multirow[t]{6}{*}{ Cathodal } & $\operatorname{MEP}(\mathrm{mV})$ & PLC & $1.03 \pm 0.14$ & $0.99 \pm 0.12$ & $1.01 \pm 0.09$ & 0.517 \\
\hline & & Acute RBX & $1.01 \pm 0.13$ & $0.97 \pm 0.09$ & $0.99 \pm 0.08$ & 0.946 \\
\hline & & Chronic RBX & $0.94 \pm 0.06$ & $1.03 \pm 0.12$ & $1 \pm 0.09$ & 0.063 \\
\hline & $\% \mathrm{MSO}$ & PLC & $52.9 \pm 7.54$ & $52.9 \pm 7.54$ & $53.3 \pm 8.09$ & 0.801 \\
\hline & & Acute RBX & $51.6 \pm 6.19$ & $51.6 \pm 6.19$ & $51.3 \pm 7.43$ & 0.163 \\
\hline & & Chronic RBX & $51.9 \pm 7.62$ & $51.9 \pm 7.62$ & $51.3 \pm 7.6$ & 0.096 \\
\hline
\end{tabular}

Shown are the mean MEP amplitudes \pm S.D. and stimulation intensity (percentage of maximum stimulator output, \%MSO) mean \pm S.D. of baseline 1,2 , and 3 . The intensity of TMS was determined to elicit MEPs with a peak to peak amplitude of $\sim 1 \mathrm{mV}$ (baseline1). A second baseline (baseline 2) was recorded two hours after medication intake to determine the effect of the drug on cortical excitability and adjusted if necessary (baseline 3). Student's t-tests revealed no significant differences between conditions $(\mathrm{P}>0.05)$. 
Table 2. Results of the repeated-measures ANOVA.

\begin{tabular}{lccc}
\hline \multicolumn{1}{c}{ Parameters } & df & F-value & p-value \\
\hline Drug & 2 & 7.843 & $0.006^{*}$ \\
Stimulation & 1 & 19.852 & $0.002^{*}$ \\
Time & 14 & 3.220 & 0.078 \\
Drug x Stimulation & 2 & 10.159 & $0.007^{*}$ \\
Drug x Time & 28 & 2.034 & 0.118 \\
Stimulation x Time & 14 & 4.964 & $0.005^{*}$ \\
Drug x Stimulation x Time & 28 & 1.356 & 0.125 \\
\hline
\end{tabular}

*Significant results at $\mathrm{p}<0.05$, df: degrees of freedom 


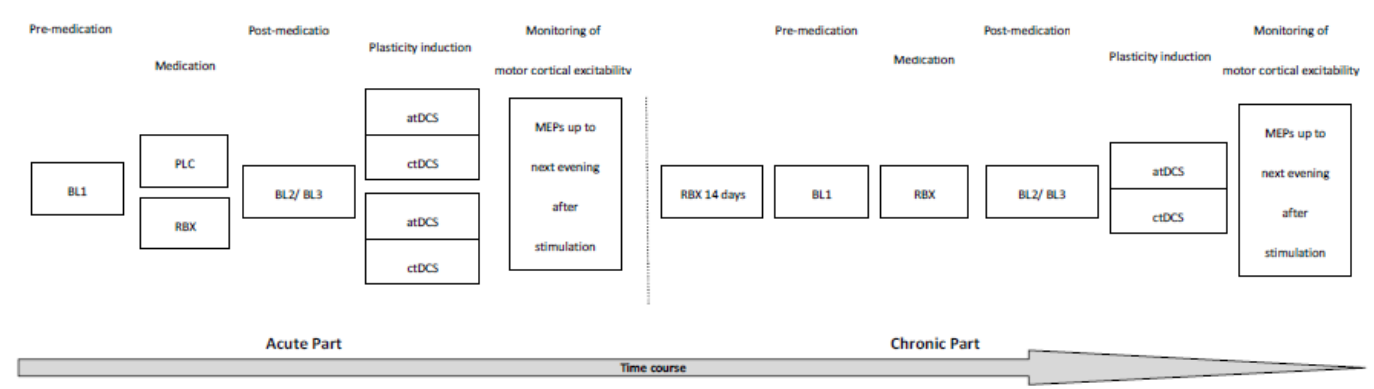

Figure 1. Experimental course of the present study.

The study was conducted in two parts. For the single dose medication part, subjects received a single dosage of $8 \mathrm{mg}$ reboxetine (RBX) or placebo medication (PLC) with anodal or cathodal tDCS. After these 4 sessions, which were separated by at least one week from each other, they took $8 \mathrm{mg}$ RBX consecutively for 21 days. In the "chronic" part of the experiments, the other two sessions (RBX with anodal or cathodal tDCS) were conducted at the end of the second and third week. For each session, first baseline MEP (BL1) were recorded with TMS. Two hours after intake of the medication, a second baseline (BL2) was determined and adjusted if necessary (BL3). Afterwards, tDCS was applied and MEPs were recorded after stimulation at different time points until next evening. 
A

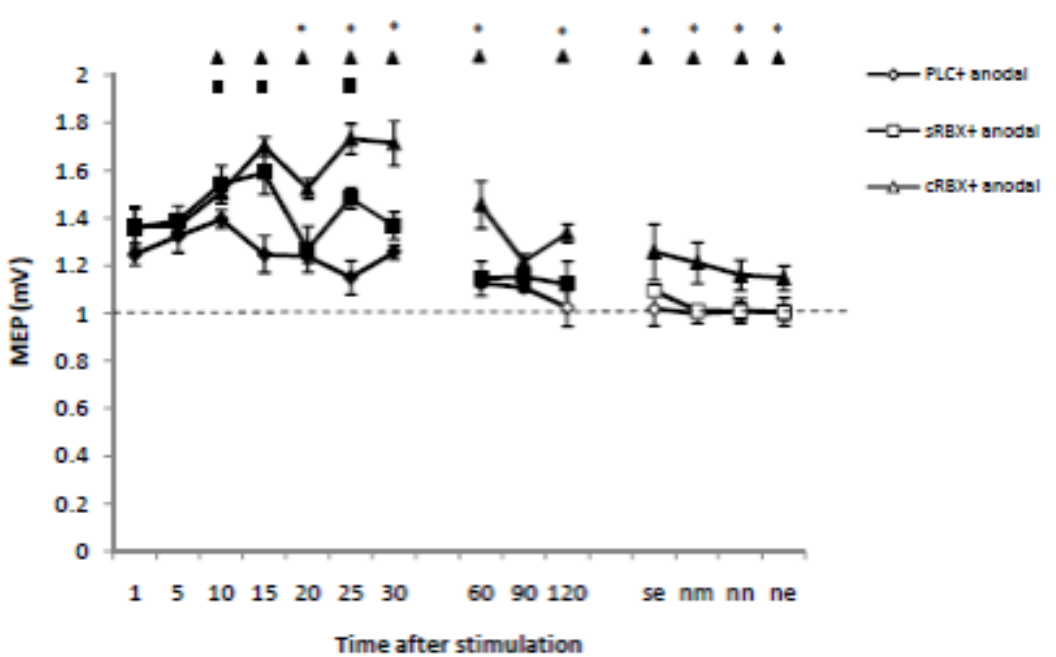

B

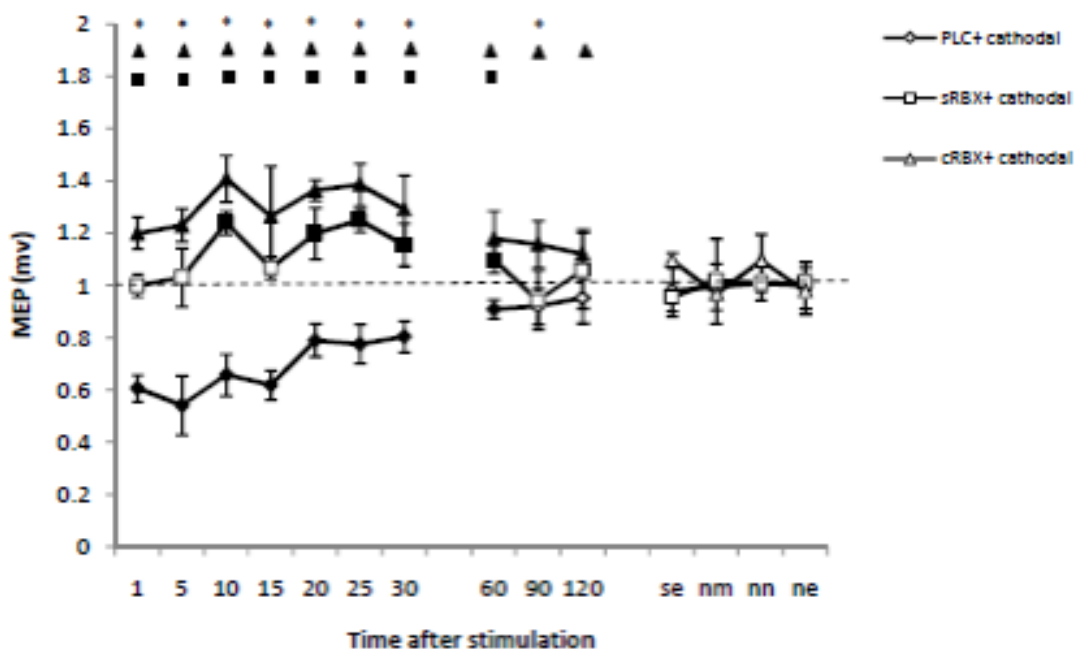

Figure 2. Impact of single dose and chronic reboxetine (RBX) on transcranial direct current stimulation (tDCS)-induced motor cortex plasticity.

Shown are raw MEP amplitudes after plasticity induction by anodal/cathodal tDCS under placebo (PLC), single dose RBX (sRBX), or chronic RBX (cRBX) conditions up to the next evening of the stimulation day. (A) In the PLC condition (diamonds), anodal tDCS induced a significant excitability enhancement for up to 90 minutes after stimulation. Single dose RBX (square) resulted in excitability enhancements for up to 120 minutes after stimulation. Chronic RBX (triangle) enhanced and prolonged these excitability enhancements until next evening. (B) In the PLC condition (diamond), cortical excitability was significantly reduced after cathodal tDCS for 60 minutes, 
whereas single dose (square) and chronic RBX (triangle) converted the inhibitory effect into facilitation. Furthermore chronic RBX showed a significant enhancement of MEP amplitudes compared to the single dose condition for 30 minutes and at the time point of 90 after stimulation. Error bars indicate S.E.M. The black symbols indicate significant differences of post-stimulation MEP amplitudes fromrespectivebaseline values; Floating symbols ( . : single dose RBX, . : chronic $\mathrm{RBX}$ ) indicate a significant difference between respective $\mathrm{RBX}$ conditions and placebo medication at the same time points (Student's t-test, two tailed, paired samples, $\mathrm{p}<0.05) . *$ indicate significant differences between acute and chronic RBX conditions at the same time points (Student's t-test, two tailed, paired samples, $\mathrm{p}<0.05)$. se $=$ same evening; $\mathrm{nm}=$ next morning; $\mathrm{nn}=$ next noon; $\mathrm{ne}=$ next evening. 


\subsection{Acute and chronic noradrenergic effects on cortical excitability in healthy}

\section{humans}

Recent studies have shown that noradrenaline affects cortical excitability and cortical activity. A single dose of the selective noradrenaline reuptake inhibitor (NRI) reboxetine (RBX) enhanced the slope of input-output curve (I-O curve) and intracortical facilitation (ICF) but showed no effect on motor thresholds (MTs) and intracortical inhibition (Plewnia et al., 2002; Plewnia et al., 2004). In principal accordance, Herwig et al. reported increased ICF, but also decreased intracortical inhibition under RBX (Herwig et al., 2002). With regard to the contribution of adrenergic receptor subtypes, following a single dose of the $\alpha$-2-adrenoreceptor agonist guanfacine, a decrease of the I-O curve and ICF as well as an increase of intracortical inhibition have been described (Korchounov et al., 2003). These findings confirm that noradrenaline is involved in human brain excitability, but the mechanism is complex, and probably receptor subtype-dependent. Clinically, therapeutic effects are usually obtained after some weeks (Kasper et al., 2000), which might go along with different physiological effects. However, the chronic impact of noradrenaline on cortical excitability has not been explored so far, and the underlying mechanism is also unclear. Therefore, the purpose of this study was to investigate the acute and chronic effects of the selective NRI RBX, on cortical excitability in healthy humans in a double-blind, placebo-controlled, randomized crossover study. Sixteen subjects were assessed with different TMS measurements: MTs, I-O curve, short-latency intracortical inhibition (SICI) and facilitation (ICF), I-wave facilitation, and short-interval afferent inhibition (SAI) before and after placebo or RBX (8mg) single dose administration. Afterwards, the same subjects took RBX (8mg/ day) consecutively for 21 days. During this period (subjects underwent two experimental 
sessions with identical TMS measures under placebo or RBX medication), TMS measurements were assessed before and after drug intake. Both single dose and chronic administration of RBX increased the slope of the I-O curve, ICF, and I-wave facilitation, but decreased SICI and SAI. Moreover, chronic RBX showed a more stable enhancement of ICF and I-wave facilitation as compared to single dose application. The results might explain possible mechanisms underlying the beneficial effect of RBX in neurological and psychiatric diseases. 


\title{
Acute and chronic noradrenergic effects on cortical excitability
}

\author{
in healthy humans
}

Hsiao-I Kuo ${ }^{12}$, Prof. Dr. Walter Paulus ${ }^{1}$, Dr. Giorgi Batsikadze ${ }^{4}$, Asif Jamil ${ }^{12}$, Dr. Min-Fang Kuo ${ }^{2}$, Prof. Dr. Michael A. Nitsche $e^{1,2,3 *}$

${ }^{1}$ Department of Clinical Neurophysiology, University Medical Center, Georg-August-University, Robert-Koch-Straße 40, 37075 Göttingen, Germany

${ }^{2}$ Dept. Psychology and Neurosciences,Leibniz Research Centre for Working Environment and Human Factors, Ardeystrasse 67, Dortmund, Germany

${ }^{3}$ Department of Neurology, University Medical Hospital Bergmannsheil, Bochum, Germany

${ }^{4}$ Department of Neurology, Essen University Hospital, University of Duisburg-Essen, Germany

*Corresponding author: Prof. Dr. M.A. Nitsche, Department Psychology and Neuroscience, Leibniz Research Centre for Working Environment and Human Factors, Ardeystrasse 67, Dortmund Germany. Tel: +4955139 9571, Fax: +49551398126. E-mail: nitsche@ifado.de

Abstract: 233 words

Manuscript: 3668 words

Number of references: 43

Table: 1

Figures: 5 


\section{Significance Statement}

- Acute and chronic enhancement of noradrenergic brain activity via single dose and chronic administration of reboxetine (RBX) increases cortical excitability via enhancement of facilitation and reduction of inhibition.

- Chronic RBX results in a more stable enhancement of intracortical facilitation and I-wave facilitation as compared to single dose application.

- Chronic RBX itself, without an additional acute loading dose, enhances corticospinal excitability and I-wave facilitation compared to placebo medication. 


\section{Abstract}

Background: Noradrenaline is a major neuromodulator in the central nervous system, and it is involved in the pathophysiology of diverse neuropsychiatric diseases. Previous transcranial magnetic stimulation (TMS) studies suggested that acute application of selective noradrenaline reuptake inhibitors (NRI) enhances cortical excitability in the human brain. However, it usually requires prolonged NRI treatment to achieve therapeutic effects in clinical populations, which might go along with different physiological effects.

Methods: The purpose of this study was to investigate the acute and chronic effects of the selective NRI reboxetine (RBX) on cortical excitability in healthy humans in a double-blind, placebo-controlled, randomized crossover study. Sixteen subjects were assessed with different TMS measurements: motor thresholds (MTs), input-output curve (I-O curve), short-latency intracortical inhibition (SICI) and intracortical facilitation (ICF), I-wave facilitation, and short-interval afferent inhibition (SAI) before and after placebo or RBX (8mg) single dose administration. Afterwards, the same subjects took RBX (8mg/ day) consecutively for 21 days. During this period (subjects underwent two experimental sessions with identical TMS measures under placebo or RBX), TMS measurements were assessed before and after drug intake. 
Results: Both single dose and chronic administration of RBX increased cortical excitability, increased the slope of the I-O curve, ICF, and I-wave facilitation, but decreased SICI and SAI. Moreover, chronic RBX showed a more stable enhancement of ICF and I-wave facilitation as compared to single dose application.

Conclusions: The results might explain possible mechanisms underlying the beneficial effect of RBX in neurological and psychiatric diseases.

Key words: reboxetine, noradrenaline, cortical excitability, transcranial magnetic stimulation 


\section{Introduction}

Noradrenaline is a neuromodulator in the central nervous system (CNS) which regulates various neuropsychological processes (Bhagya et al., 2015; Robinson, 2012). Moreover, a single dose of the selective noradrenaline reuptake inhibitor (NRI), reboxetine (RBX), can improve working memory and motor learning in healthy subjects as well as in clinical populations such as depression and stroke (Ferguson et al., 2003; Plewnia et al., 2004; Wang et al., 2009; Wang et al., 2011). The foundation for these effects might be the impact of noradrenaline on neuroplasticity and cortical excitability. Animal studies have shown that noradrenaline enhances long-term potentiation (LTP) as well as long-term depression (LTD) (Kirwood et al., 1999; Nakadate et al., 2006; Tully et al., 2007). The direction of LTP and LTD depends on the activation of $\alpha$ - and $\beta$-adrenoreceptors. Typically, activation of $\beta$-adrenoreceptors enhances LTP, whereas the activation of $\alpha$-adrenoreceptors reduces it (Kemp and Manahan-Vaughan, 2008; Marzo et al., 2009). These receptors also affect various intracellular processes such as ion channel opening via modulation of N-methyl-D-aspartate (NMDA) and gamma-aminobutyric acid (GABA) receptors (Hu et al., 2007; Tully et al., 2007).

Recently, transcranial magnetic stimulation (TMS) measures have been applied to test the effects of noradrenaline on cortical excitability in humans. Corticospinal excitability can be assessed by active and resting motor thresholds (MTs) and the input-output curve (I-O curve) (Abbruzzese and Trompetto, 2002; Chen, 2000). MTs reflect neuronal membrane excitability and depend primarily on ion channel activity, as they are increased by voltage-gated sodium channel blockers, but not affected by 
drugs modulating GABAergic or glutamatergic transmission (Ziemann et al., 1998a; Ziemann et al., 1996). The input-output curve (I-O curve) serves as an index of excitability of larger neuronal populations compared to MTs (Abbruzzese and Trompetto, 2002; Chen, 2000). The I-O curve depends on neuronal membrane excitability, because its slope is decreased by sodium and calcium channel blockers. Furthermore, synaptic mechanisms are involved as it is modulated by drugs influencing the GABAergic and glutamatergic system (Broojerdi et al., 1999; Lazzaro et al., 2003; Paulus et al., 2008). Short-latency intracortical inhibition (SICI) and intracortical facilitation (ICF), and motor cortex indirect waves (I-waves) are studied by paired-pulse TMS. SICI is mainly influenced by glutamate and $\mathrm{GABA}_{\mathrm{A}}$ receptors and based on the induction of inhibitory postsynaptic potentials (Liepert et al., 1997; Ziemann et al., 1998a; Ziemann et al., 1996). ICF is thought to reflect activity of GABAergic and glutamatergic systems. I-waves are thought to be primarily controlled by GABA-related neuronal circuits (Ziemann et al., 1998a; Ziemann et al., 1998b). In previous studies, a single dose of RBX enhanced the slope of I-O curve and ICF but showed no effect on MTs and intracortical inhibition (Plewnia et al., 2004; Plewnia et al., 2002). Herwig et al. also reported increased ICF, but also decreased intracortical inhibition under RBX (Herwig et al., 2002). With regard to the contribution of adrenergic receptor subtypes, following a single dose of the $\alpha$-2-adrenoreceptor agonist guanfacine, a decrease of the I-O curve and ICF as well as an increase of intracortical inhibition have been described (Korchounov et al., 2003). Thus, the RBX-induced excitability enhancement, which differs relevantly from the effect of $\alpha$-2-adrenoreceptor activation, might be primarily driven by excitatory effects of $\beta$-adrenoreceptors. These findings confirm that noradrenaline is involved in human brain excitability, but the mechanism is complex, and probably receptor 
subtype-dependent. The above-mentioned pharmacological TMS studies were conducted via single dose protocols. In clinical applications, therapeutic effects are usually obtained after some weeks (Kasper et al., 2000). It might be speculated that the physiological effects of the respective substances differ between single dose and chronic treatment. Therefore, we aimed to compare the effects of single dose with chronic treatment of RBX on motor cortex excitability in healthy volunteers, using a variety of single and paired-pulse TMS measures tackling different ion channels and receptors. In accordance with previous studies, we hypothesized that a single dose of RBX enhances cortical excitability in healthy humans. According to the superior clinical results obtained from chronic treatment, we furthermore hypothesized that chronic application might also lead to more pronounced enhancement of cortical excitability compared to a single dose application.

\section{Method}

\section{Subjects}

Sixteen healthy subjects ( 8 females) aged between $27.5 \pm 4.01$ years (mean \pm standard deviation) were recruited. Subjects were all right-handed, between 18 and 50 years old, and currently non-pregnant. None of them had a history of neurological diseases, electric implants in the body, or took other medications during the study period. Written informed consent was obtained from all subjects who participated in the study before inclusion. The study was approved by the Ethics Committee of the University of Göttingen, and conformed to the Declaration of Helsinki.

Pharmacological intervention 
8mg reboxetine (RBX) or an equivalent placebo (PLC) drug was administered 2 hours before the start of each experimental session, allowing the verum substance to induce a stable plasma level and prominent effects in the central nervous system (Dostert et al., 1997; Pellizzoni et al., 1996). Steady state plasma concentrations are achieved after five days of drug intake, and clinically, the majority of antidepressants have therapeutic effects after approximately 2 weeks of treatment (Dostert et al., 1997; Kasper et al., 2000). Thus, for the chronic RBX condition, we designated a three week period of RBX intervention, and started to measure cortical excitability after 2 weeks of application.

Monitoring of motor cortical excitability

Motor evoked potentials (MEPs) were induced in the right abductor digiti minimi muscle (ADM) by single-pulse TMS over the left primary motor cortex, conducted by a Magstim 200 magnetic stimulator (Magstim Company, Whiteland, Dyfed, United Kingdom) with a figure-of-eight magnetic coil (diameter of one winding $=70 \mathrm{~mm}$; peak magnetic field=2.2 T). For the paired-pulse TMS protocols, the coil was connected to two Magstim 200 stimulators via a bistim module. The coil was held tangentially to the skull, with the handle pointing backwards and laterally at $45^{\circ}$ from midline. The optimal position was defined as the site where TMS resulted consistently in the largest MEP. Surface electromyography (EMG) was recorded from the right ADM by use of $\mathrm{Ag}-\mathrm{AgCl}$ electrodes in a belly tendon montage. The signals were amplified, and band-pass filtered ( $2 \mathrm{~Hz}$ to $2 \mathrm{kHz}$; sampling rate, $5 \mathrm{kHz}$ ). Signals were digitized with a power 1401 data acquisition interface (Cambridge Electronic Design, Cambridge, United Kingdom) and stored for offline analysis. 
Motor threshold determination

The resting motor threshold (RMT) was defined as the minimum TMS intensity which elicited a peak-to-peak MEP of $50-100 \mu \mathrm{V}$ in the relaxed muscle in at least three of six consecutive trials. The active motor threshold (AMT) was the minimum intensity eliciting a MEP response of $\sim 200-300 \mu \mathrm{V}$ during moderate spontaneous background muscle activity ( $\sim 15 \%$ of the maximum muscle strength) in at least three of six consecutive trials.

Input-output curve (I-O curve)

The I-O curve was determined using TMS intensities of 100, 110, 130, and 150\% RMT (15 stimuli per block (each intensity), with the order of the blocks randomized).

Short-latency intracortical inhibition and intracortical facilitation (SICI-ICF)

Short-latency intracortical inhibition and facilitation were measured by a TMS paired-pulse protocol including ISIs of 2, 3, 5, 10, and $15 \mathrm{~ms}$. The first three ISIs represent inhibitory, and the last two ISIs reveal facilitatory effects, which reflect excitability of inhibitory and excitatory interneurons, respectively (Kujirai et al., 1993). In this protocol, the subthreshold conditioning stimulus (determined as $70 \%$ of AMT) precedes the test stimulus. The test pulse was adjusted to achieve a baseline MEP of $\sim 1 \mathrm{mV}$ and readjusted during the respective stimulation protocols, if needed, to compensate for effects of global excitability changes on test-pulse amplitude. The pairs of stimuli were organized in 15 blocks, where each ISI was represented once together with one additional single test pulse in a pseudo-randomized order for each block. 
I-wave facilitation

I-wave facilitation was investigated using a TMS paired-pulse protocol including ISIs of $1.1,1.3,1.5,2.3,2.5,2.7,2.9,4.1,4.3$ and $4.5 \mathrm{~ms}$ (Ziemann et al., 1998b). In this protocol, the TMS test stimulus precedes the conditioning stimulus (determined as $70 \%$ of RMT). The test pulse was adjusted to achieve a baseline MEP of $\sim 1 \mathrm{mV}$ and readjusted during the respective stimulation protocols, if needed, to compensate for effects of global excitability changes on test-pulse amplitude. The pairs of stimuli were organized in blocks in which each ISI and one test pulse was represented once and was pseudo-randomized. These blocks were repeated 15 times.

\section{Short-interval afferent inhibition (SAI)}

SAI combines peripheral and motor cortex stimulation to evaluate activity of cholinergic systems in the human brain (Lazzaro et al., 2006). In this protocol, a suprathreshold electric pulse (width of $200 \mu$ s and an intensity of $200 \%$ of the perceptual threshold) over the ulnar nerve precedes the motor cortex TMS test pulse. The test pulse was adjusted to achieve a baseline MEP of $\sim 1 \mathrm{mV}$ and readjusted during the respective stimulation protocols, if needed, to compensate for effects of global excitability changes on test-pulse amplitude. Peripheral nerve stimulation was delivered by a Digitimer D185 stimulator (Digitimer Ltd., Welwyn Garden City, United Kingdom). Interstimulus intervals of 20 and $40 \mathrm{~ms}$ between the peripheral and cortical stimulus were used (Lazzaro et al., 2006). The control-conditioning test pairs and a single TMS pulse control condition were recorded 20 times in random order.

\section{Experimental procedures}

The study was divided into two parts, each with two experimental sessions. Within 
each part, sessions were carried out in randomized order and separated by at least one week to avoid cumulative drug effects. All volunteers completed both parts of the study. The first part explored single dose RBX effects. Subjects were seated in a comfortable chair with head and arm rests. The right ADM hotspot was determined over the left primary motor cortex, and 20 MEPs were recorded with the TMS intensity which elicited an average $1 \mathrm{mV}$ MEP $\left(\mathrm{SI}_{1 \mathrm{mV}}\right)$. Afterwards, RMT and AMT were determined using standard procedures. After measuring AMT, a 15 min break followed to avoid a possible effect of muscle contraction on the next measurements. After this break, the following parameters were recorded in randomized order as baseline measures: I-O curve, SICI-ICF, I-wave facilitation and SAI. Afterwards, the participants took single-dose placebo (sPLC) or RBX (sRBX). Two hours after medication, TMS was readjusted to obtain single test pulse amplitudes of $1 \mathrm{mV}$, if needed. Then all of the above-mentioned parameters were measured again. The second part of the experiment explored chronic RBX effects. The same participants received RBX (8mg/day) consecutively for 21 days. During this period, the other two sessions with RBX (chronic RBX condition (cRBX): with $\mathrm{RBX}$ at the day of experiment) or placebo (chronic placebo condition (cPLC): with placebo at the day of experiment) were conducted in randomized order at the end of the second and third week after the start of chronic drug intake. The procedure in each session was the same as in the first part of the study (Figure 1).

Data analysis

With regard to the RMT, AMT and $\mathrm{SI}_{1 \mathrm{mV}}$, the individual means of the TMS intensity at RMT, AMT and $\mathrm{SI}_{1 \mathrm{mV}}$ were calculated for the before and after drug administration 
conditions separately. Repeated-measures analyses of variance (ANOVA) were performed for the above-mentioned data using the RMT, AMT, and $\mathrm{SI}_{1 \mathrm{mV}}$ value as the dependent variable, and drug condition (sPLC, sRBX, cRBX, and cPLC) and time point (pre- and post- medication) as independent within-subject factors. For significant ANOVA results, exploratory post hoc comparisons were performed using Student's t-tests (paired samples, two tailed, $\mathrm{p}<0.05$, not corrected for multiple comparisons).

For the I-O curve, the individual means of MEP amplitudes were calculated for all subjects. Repeated-measures ANOVAs were performed on the above-mentioned data using MEP amplitudes as the dependent variable, and drug condition (sPLC, sRBX, cRBX, and cPLC), time point (pre- and post- medication), and TMS intensity as independent within-subject factors. For significant ANOVA results, exploratory post hoc comparisons were performed using Student's t-tests (paired samples, two tailed, $\mathrm{p}<0.05$, not corrected for multiple comparisons).

Regarding SICI-ICF, I-wave facilitation, and SAI, the mean values were normalized to the respective single-pulse condition. First, intra-individual means were calculated for each condition. To determine significance, repeated measures ANOVAs were performed (ISIs, drug conditions, and time point as independent within-subject factors and MEP amplitude as the dependent variable). In case of significant results of the ANOVA, exploratory post hoc comparisons were performed using Student's t-tests (paired samples, two tailed, $\mathrm{p}<0.05$, not corrected for multiple comparisons).

\section{Results}


In follow-ups, all subjects tolerated TMS and RBX without side effects (one reported a mild tingling sensation during TMS, but did finish the whole session). For RMT, AMT, and $\mathrm{SI}_{1 \mathrm{mV}}$, the respective ANOVAs resulted in no significant main effects of drug and time or the respective interactions (all p>0.05) (Table 1).

Input-output curve (I-O curve)

As displayed in Table 1, the ANOVA showed significant main effects of drug $(\mathrm{F}(3)=4.916 ; \mathrm{p}=0.006)$, intensity $(\mathrm{F}(3)=123.338 ; \mathrm{p}<0.001)$ and drug $\mathrm{x}$ intensity interaction $(\mathrm{F}(9)=4.869 ; \mathrm{P}<0.001)$. This is due to enhanced MEP amplitudes caused by increased TMS stimulator output (100, 110, 130 and 150\% RMT), and effects of different drug conditions on MEPs. As shown in Figure 2A, post hoc Student's t-tests (paired, two-tailed, $\mathrm{p}<0.05$ ) showed significantly larger MEP amplitudes elicited by 110, 130, and $150 \%$ RMT TMS intensity in the sRBX_post compared to the sPLC_post condition. Application of chronic RBX showed significantly larger MEP amplitudes compared to the cPLC_post condition in the 110, 130, and $150 \%$ RMT TMS intensity (Figure 2B). Furthermore, cRBX_post significantly enhanced the MEP amplitude compared to the sRBX_post condition in the 110 and 150\% RMT TMS conditions (Figure 2C). As compared to the sPLC_post condition, MEP amplitudes in the cPLC_post condition were significantly larger for 130 and $150 \%$ RMT, which reflects the long-lasting impact of chronic RBX application on cortical excitability (Figure 2D).

Short-latency intracortical inhibition and intracortical facilitation (SICI-ICF)

The ANOVA (Table 1) showed significant effects of drug $(\mathrm{F}(3)=8.423 ; \mathrm{P}<0.001)$, 
time $(\mathrm{F}(1)=9.489 ; \mathrm{P}=0.01)$, ISI $(\mathrm{F}(4)=5.171 ; \mathrm{p}=0.002)$, drug $\mathrm{x}$ time $(\mathrm{F}(3)=13.770$; $\mathrm{p}<0.001)$, drug $x$ ISI $(F(12)=4.481 ; \mathrm{p}<0.001)$, time $x$ ISI $(F(4)=5.831 ; p=0.001)$, and drug $\mathrm{x}$ time $\mathrm{x}$ ISI interaction $(\mathrm{F}(12)=5.267 ; \mathrm{p}=0.001)$. Post hoc Student's t-tests (paired, two-tailed, $\mathrm{p}<0.05$ ) showed that both single dose and chronic RBX shifted cortical excitability towards an enhancement of excitability. As shown in Figure 3A, sRBX_post significantly increased facilitation at ISI of $15 \mathrm{~ms}$ and decreased inhibition at ISIs of 2, 3 and $5 \mathrm{~ms}$ compared to the sPLC_post condition. Administration of chronic RBX resulted in significantly enhanced MEP amplitudes at ISIs of 2, 5, 10 and $15 \mathrm{~ms}$ compared to cPLC_post condition (Figure 3B). Moreover, a stronger facilitation at ISIs of 10, and $15 \mathrm{~ms}$ and decrease of inhibition at ISIs of 2 and $5 \mathrm{~ms}$ was observed under cRBX_post compared to sRBX_post (Figure 3C). As compared to the sPLC_post condition, the cPLC_post condition showed a non-significant trend torwards enhanced facilitation and decreased inhibition (Figure 3D).

I-wave facilitation

The ANOVA (Table 1) revealed significant effects of drug $(F(3)=3.254 ; p=0.034)$, ISI $(F(9)=14.340 ; p<0.001)$, drug $x$ ISI $(F(27)=2.233 ; p<0.001)$, and drug $x$ Time $x$ ISI interaction $(\mathrm{F}(27)=1.767 ; \mathrm{p}=0.012)$. The results are caused by significant enhancement of I-wave facilitation under both single dose and chronic RBX conditions. As shown in Figure 4A, application of single dose RBX significantly enhanced MEP amplitudes at all ISI compared sPLC_post condition. Similarly, cRBX_post significantly enhanced I-wave facilitation at nearly all ISIs compared to sPLC_post condition (Figure 4B). Administration of chronic RBX revealed a significantly enhanced facilitation at nearly all ISIs compared to the sRBX_post condition (Figure 4C). In addition, as compared to the sPLC_post condition, the 
cPLC_post condition demonstrated significantly increased facilitation at all ISI (Figure 4D).

Short-interval afferent inhibition (SAI)

The ANOVA revealed significant main effects of drug $(F(3)=19.886 ; p<0.001)$, time $(\mathrm{F}(1)=14.218 ; \mathrm{p}=0.003)$, and ISI $(\mathrm{F}(1)=32.029 ; \mathrm{p}<0.001)$ (Table 1). Accordingly, after application of single dose and chronic RBX, SAI was significantly decreased at ISI $20 \mathrm{~ms}$. As compared to the sPLC condition, the cPLC condition demonstrated significant decreased SAI at ISI 20ms after drug intake. However, both, single dose and chronic RBX did not have any significant impact on SAI at an ISI of $40 \mathrm{~ms}$ (Figure 5).

\section{Discussion}

In the present study, we explored the effects of single dose and chronic noradrenaline enhancement on cortical excitability in healthy subjects by different TMS protocols (corticospinal excitability: MTs and I-O curves; intracortical excitability: SICI-ICF, I-wave facilitation, and SAI). In general, our findings show enhanced corticospinal excitability, intracortical facilitation, and I-wave facilitation, but reduced intracortical inhibition and SAI after single dose and chronic RBX intake. Furthermore, intracortical facilitation and I-wave facilitation were more prominently enhanced in the chronic medication condition. These findings might partially explain why the onset of action of antidepressants takes several weeks in clinical application (Anderson et al., 2000; Heinbotham and Dunwiddie, 1991). Additionally, chronic RBX itself without an additional acute loading dose enhanced facilitation of I-O curve 
and I-wave facilitation compared to placebo medication, which shows that chronic application of RBX itself results in prolonged excitability alteration.

Regarding corticospinal excitability, AMT, RMT as well as $\mathrm{SI}_{1 \mathrm{mV}}$ did not differ between single dose RBX, chronic RBX, and placebo medication. However, I-O curve MEP amplitudes were enhanced after both single dose and chronic RBX. Animal studies showing that noradrenaline enhances glutamatergic facilitation in the hippocampus might explain these results, as higher TMS intensities of the I-O curve are affected by glutamatergic mechanisms (Hu et al., 2007). Moreover, our results are in line with previous human studies which found that single dose RBX and the presynaptic $\alpha 2$-antagonist yohimbine increase the slope of I-O curve but do not alter MTs (Plewnia et al., 2001; Plewnia et al., 2004; Plewnia et al., 2002). Since measuring the I-O curve involves larger neuronal populations as compared to MTs, the sensitivity of the I-O curve in detecting cortical excitability changes might be superior. This - beyond the impact of glutamatergic mechanisms on the I-O curve might be another reason why the I-O curves, but not MTs, are modulated by RBX. As the enhancement of the I-O curve originates at cortical or/and subcortical sites, our findings indicate that modulation of central noradrenaline is a possible way to enhance cortico-spinal excitability.

SICI-ICF and I-wave facilitation were prominently modulated by RBX, especially in the chronic application mode. Here, we found enhanced ICF and I-wave facilitation as well as a decreased inhibition after RBX administration. ICF is regulated predominantly by glutamate receptors with some $\mathrm{GABA}_{\mathrm{A}}$ receptor contribution (Ziemann et al., 1998a; Ziemann et al., 1995; Ziemann et al., 1998b). SICI and I-wave 
facilitation are suggested to be primarily controlled by GABAA receptors (Ilic et al., 2002; Paulus et al., 2008; Ziemann et al., 1998b). Our results thus imply that noradrenaline exerts modulation effects on both, excitatory glutamatergic neurotransmission and GABA-related inhibition. These results are in accordance with those of former in-vivo and in-vitro studies showing that noradrenaline suppresses GABAergic inhibition and enhances glutamatergic facilitation (Hu et al., 2007; Tully et al., 2007). Furthermore, studies in humans have shown that single dose RBX decreases SICI and increases ICF (Herwig et al., 2002; Plewnia et al., 2004; Plewnia et al., 2002). These findings however contrast with one study, in which application of RBX for 16 days did not affect ICI and ICF (Lange and Liepert, 2007). A possible explanation might be the longer duration of drug intake in our study, which might result in more prominent effects.

Since SAI is directly controlled by the cholinergic system, modulation of the noradrenergic system might have decreased SAI by cholinergic activation (Lazzaro et al., 2000). In the visual cortex, slice studies have shown that noradrenaline acts in combination with the cholinergic system, which results in facilitation of LTP (Brocher et al., 1992). However, a contribution of the GABAergic system to the RBX effects cannot be ruled out at present, as SAI is also affected by GABAergic mechanisms (Lazzaro et al., 2007), and other results of our study are in accordance with a prominent impact of RBX on GABA activity.

\section{Mechanisms}

The specific mechanisms responsible for the effects of RBX on cortical excitability in the human brain should be further investigated in forthcoming studies. One possible 
mechanism is the reduction of potassium conductance by RBX (Hass and Konnerth, 1983). This would result in a depolarization of postsynaptic membranes and enhance calcium influx into the intraneuronal compartment through NMDA receptors and voltage-dependent calcium channels, which might enhance cortical facilitation $(\mathrm{Gu}$, 2002). The $\beta$-adrenoreceptor might be the candidate involved in this mechanism as activation of $\beta$-adrenoreceptors decreases potassium conductance and results in depolarization of postsynaptic neurons (Hass and Konnerth, 1983). Moreover, the main mechanism suggested by the results of our experiments, an increase of glutamatergic and reduction of GABAergic activity by noradrenergic enhancement, is supported by recent animal studies showing that $\beta$-adrenoreceptors suppress GABAergic inhibition and facilitate activation of NMDA receptors in different brain areas (Hu et al., 2007; Tully et al., 2007).

\section{Functional relevance}

Our finding is in accordance with a previous study of our group in which it was shown that RBX prolongs and enhances LTP-like plasticity induced by non-invasive brain stimulation (unpublished observations). Additionally, recent studies have shown that increased excitability may be relevant for functional adaptation of neuronal networks in neuropsychiatric diseases (Henn and Vollmayer, 2004; Holderbach et al., 2007; Nudo et al., 2001), which is in line with studies showing that noradrenergic agents improve cognitive processes such as working memory and motor learning in healthy and depressed subjects (Ferguson et al., 2003; Wang et al., 2011). Thus RBX as a selective NRI might have long term therapeutic effects by enhancing cortical excitability. 


\section{Limitations}

Some potential limitations of the present study should be taken into account. First, the study was conducted in healthy subjects. In neuropsychiatric diseases, transmitter availability and other features of brain function might be different; future studies are thus needed to explore transferability of these results to patients. Moreover, the impact of noradrenaline on excitability based on dosages and receptor subtype contributions should be explored in future experiments. Finally, given the prominent effect of noradrenergic receptor activation on cortical excitability, future studies should explore the relevance of noradrenergic activation on functional outcomes in humans. 


\section{References}

Abbruzzese G, Trompetto C. (2002). Clinical and research methods for evaluating cortical excitability. J Clin Neurophysiol19, 307-321.

Anderson I, Nutt D, Deakin J. (2000). Evidence-based guidelines for treating depressive disorders with antidepressants: a reversion of the 1993 Britisch Association for Psychopharmacology guidelines. Journal of Psychopharmacology14, 3-20.

Bhagya V, Srikumar B, Traju T, Rao B. (2015). The selective noradrenergic reuptake inhibitor reboxetine restores spatial learning deficits, biochemical changes, and hippocampal synaptic plasticity in an animal model of depression. Jounal of Neuroscience Research93, 104-120.

Brocher S, Artola A, Singer W. (1992). Agonist of cholinergic and noradrenergic receptors facilitate synergistically the induction of long-term potentiation in slices of rat visual cortex. Brain Res.573, 27-36.

Broojerdi V, Battaglia F, Meullenbacher W, Cohen L. (1999). Evaluation of the effects of CNS-active drugs on cortical excitability in intact humans. Neurology52, 457.

Chen R. (2000). Studues of human motor physiology with transcrnial magnetic stimulation. Muscle Nerve Suppl9, 26-32.

Dostert P, Benedetti M, Poggesi I. (1997). Review of the pharmacokinetics and metabolism of reboxetine, a selective noradrenaline reuptake inhibitor. European Neuropsychopharmacology1, 23-35.

Ferguson J, Wesnes K, Schwartz G. (2003). Reboxetine versus paroxetine versus placebo: effects on conitive functioning in depressed patients. International Clinical Psychopharmacology18, 9-14.

Gu Q. (2002). Neuromodulatory transmitter systems in the cortex and their role cortical plasticity. Neuroscience111, 815-835.

Hass H, Konnerth A. (1983). Histamine and noradrenaline decrease calcium-activated potasium conductance in hippocampal pyramidal cells. Nature302, 432-434.

Heinbotham L, Dunwiddie T. (1991). Long-term increases in the evoked population spike in the CA1 region of rat hippocampal pyramidal cells. Nature302, 432-434.

Henn F, Vollmayer B. (2004). Basic pathophysiological machanisms in depression: what are they and how might they affect the course of the illness? . Pharmacopsychiatry37, 152-156.

Herwig U, Brauer K, Connemann B, Spitzer M, Schonfeldt-Lecuona C. (2002). Intracortical excitability is modulated by a norepinephrine-reuptake inhibitor sa measured with piared-pulse transcrnial magnetic stimulation. Psychopharmacology164, 228-232.

Holderbach R, Clark K, Moreu J, Bischofberger J, Norman C. (2007). Enhanced long-term synaptic depression in an animal model of depression. Biol Psychiatry62, 373-380.

Hu H, Real E, Takamiya K, Kang M, Ledoux J, Huganir RL, Malinow R. (2007). Emotion enhances learning via norepinephrine regulation of AMPA-receptor trafficking. Cell131, 160-173.

Ilic T, Meintschel F, Cleff U, Ruge D, Kessleer K, Ziemann U. (2002). Short-interval paired-pulse inhibition and facilitation of human motor cortex: 
the dimension of stimulation intensity. . J Physiol545, 771-782.

Kasper S, Giamal N, Hilger E. (2000). Reboxetine: the first selective noradrenaline re-uptake inhibitor. Exp Opin Pharmacother1, 771-782.

Kemp A, Manahan-Vaughan D. (2008). Beta-adrenoreceptors comprise a critical element in learning-facilitated long-term platicity. Cerebral Cortex18, 1326-1334.

Kirwood A, Rozas C, Kirwood J, Perez F, Bert M. (1999). Mosulation of long-term synaptic depression in visual cortex by acetylcholine and norepinephrine. $J$ Neurosci19, 1599-1609.

Korchounov A, Ilic T, Ziemann U. (2003). The alpha 2-adrenergic agonist guanfacine reduces excitability of human motor cortex through disfacilitation and increase of inhibition. Clinical Neurophysiol114, 1834-1840.

Kujirai T, Caramia M, Rothwell J, Day B, Thompson P. (1993). Cortical inhibition in human motor cortex. $J$ Physiol471, 501-519.

Lange R, Liepert W. (2007). Chronic dose effects of reboxetine on motor skill acquisition and cortical excitability. Journal of Neural Transmission114, 1085-1089.

Lazzaro V, Olivero A, Profice P, Pennisi M, Giovanni S, Zito G, Tonali P, Rothwell JC. (2000). Muscarinic receptor blockade has differential effects on the excitability of intracortical circuits in the human motor cortex. Exp Brain Res135, 455-461.

Lazzaro V, Oliviero A, Profice P, Pennisi M, Pilato F, Zito G, Dileone M, Nicoletti R, Pasqaletti P, Tonali PA. (2003). Ketamine increases human motor cortex excitability to transcranial magnetic stimulation. J Physiol547, 485-496.

Lazzaro V, Pilato F, Dileone M, Profice P, Ranieri F, Ricci V, et al. (2007). Sergergating two inhibitory circuits in human motor cortex at the level of GABA $_{A}$ receptor subtypes: a TMS syudy. Clin Neurophysiol118, 2207-2214.

Lazzaro V, Pilato F, Dileone M, Saturno E, Oliviero A, Marra C, Ranieri F, Quaranta D, Gainotti G, Tonali PA. (2006). In vivo cholinergic circuit evaluation in frontotemporal and Alzheimer dementias. Neurology66, 1111-1113.

Liepert J, Schwenkreis P, Tagenhoff M, Malin J. (1997). The glutamate antagonist riluzole suppresses intracortical facilitation. J Neurol Transm104, 1207-1214.

Marzo A, Bai J, Otani S. (2009). Neuroplasticity regulation by noradrenaline in mammalian brain. Current Neuropharmacology7, 286-295.

Nakadate K, Matsukawa M, Okado N. (2006). Identification of adrenoreceptor subtype-medisted changes in the density of synapses in the rat visual cortex Neuroscience 138, 37-46.

Nudo R, Plautz E, Frost S. (2001). Role of adaptive plasticity in recovery of function after damage to motor cortex. Muscle Nerve24, 1000-1009.

Paulus W, Classen J, Cohen L, Lang C, Lazarro V, Nitsche M, Pascaul-Leone A, Rosenow F, Rothwell JC, Ziemann U . (2008). State of the art: Pharmacologic effects on cortical excitability measures tested by transcrnial magnetic stimulation. Brain Stimul1, 151-163.

Pellizzoni C, Poggesi I, Jørgensen N, Edwards D, Paus E, Benedetti M. (1996). Pharmacokinetics of reboxetine in healthy volunteers. Single against repeated oral doses and lack of enzymatic alterations. Biopharm Drug Dispos17, 623-633.

Plewnia C, Classen J, Cohen L, Gerloff C. (2001). Noradrenergic modulation of human cortical excitability by the presynaptic alpha2-antagonist yohimbine. 
Neuroscience letters307, 41-44.

Plewnia C, Hoppe J, Cohen L, Gerloff C. (2004). Improved motor skill acquisition after selective stimulation of central norepinephrine. Neurology62, 2124-2126.

Plewnia C, Hoppe J, Heimke C, Bartles M, Cohen L, Gerloff C. (2002). Enhancement of human cortico-motoneuronal excitability by the selective norepinephrine reuptake inhibitor reboxetine. Neuroscience letters330, 231-234.

Robinson E. (2012). Blockade of noradrenaline re-uptake improves accuracy and impulse control in rat performing a five-choixe serial reaction time tasks. Psychopharmacology219, 303-312.

Tully K, Y L, Tsvetkov E, Bolshakov V. (2007). Norepinephrine enables the induction of associative long-term potentiation at thalamo-amygdala synapses. Pro Natl Acd Sci USA104, 14146-14150.

Wang L, Fink G, Dafotakis M, Grefkes C. (2009). Noradrenergic stimulation and motor performance: different effects of reboxetine on movement kinetics and visuiomotor abilities in healthy human subjects. Neuropsychologia47, 1302-1312.

Wang L, Fink G, Diekhoff S, Rehme A, Eickoff S, Grefke C. (2011). Noradrenergic enhancement improves motor network connectivity in stroke patients. ANNALS of Neurology69, 375-388.

Ziemann U, Chen R, Cohen L, Hallet M. (1998a). Dextromethorphane decreases the excitability of the human motor cortex. Neurology51, 1320-1324.

Ziemann U, Loennecker S, Paulus W. (1995). Inhibition of human motor cortex by ethanol. A transcrnial magnetic stimulation study. Brain118, 1437-1446.

Ziemann U, Loennecker S, Steinhoff B, Paulus W. (1996). Effects of antiepileptic drugs on motor cortex excitability in humans: a transcrnial magnetic stimulation study. Ann Neurol40, 367-378.

Ziemann U, Tergau F, Wassermann E, Wischer S, Hildebrandt J, Paulus W. (1998b). Demonstartion of facilitatory I wave interaction in the human motor cortex by paired transcranial magnetic stimulation. J Physiol511, 181-190. 
Table 1. Repeated-measures ANOVA results

\begin{tabular}{|c|c|c|c|c|}
\hline Measurement & Parameters & df & F-value & p-value \\
\hline \multirow[t]{3}{*}{ 1-mV intensity } & Drug & 3 & 0.358 & 0.784 \\
\hline & Time & 1 & 0.541 & 0.483 \\
\hline & Drug x Time & 3 & 1.583 & 0.220 \\
\hline \multirow[t]{3}{*}{ AMT } & Drug & 3 & 2.663 & 0.071 \\
\hline & Time & 1 & 1.583 & 0.224 \\
\hline & Drug $\mathrm{x}$ Time & 3 & 1.254 & 0.112 \\
\hline \multirow[t]{3}{*}{ RMT } & Drug & 3 & 0.534 & 0.663 \\
\hline & Time & 1 & 1.254 & 0.295 \\
\hline & Drug $x$ Time & 3 & 0.544 & 0.486 \\
\hline \multirow[t]{7}{*}{ I-O curve } & Drug & 3 & 4.916 & 0.006 \\
\hline & Time & 1 & 3.104 & 0.104 \\
\hline & Intensity & 3 & 123.338 & $<0.001$ \\
\hline & Drug $x$ Time & 3 & 1.762 & 0.174 \\
\hline & Drug x Intensity & 9 & 4.869 & $<0.001$ \\
\hline & Time $\mathrm{x}$ Intensity & 3 & 1.230 & 0.314 \\
\hline & Drug $x$ Time $\mathrm{x}$ Intensity & 9 & 1.159 & 0.330 \\
\hline \multirow[t]{7}{*}{ SICI-ICF } & Drug & 3 & 8.423 & $<0.001$ \\
\hline & Time & 1 & 9.489 & 0.01 \\
\hline & ISI & 4 & 5.171 & 0.002 \\
\hline & Drug $\mathrm{x}$ Time & 3 & 13.770 & $<0.001$ \\
\hline & Drug x ISI & 12 & 4.481 & $<0.001$ \\
\hline & Time $\mathrm{x}$ ISI & 4 & 5.831 & 0.001 \\
\hline & Drug $x$ Time $x$ ISI & 12 & 5.267 & 0.001 \\
\hline \multirow[t]{2}{*}{ I-wave facilitation } & Drug & 3 & 3.254 & 0.034 \\
\hline & Time & 1 & 2.308 & 0.157 \\
\hline
\end{tabular}




\begin{tabular}{lccc} 
ISI & 9 & 14.340 & $<\mathbf{0 . 0 0 1}$ \\
Drug x Time & 3 & 1.820 & 0.163 \\
Drug x ISI & 27 & 2.233 & $<\mathbf{0 . 0 0 1}$ \\
Time x ISI & 9 & 0.863 & 0.561 \\
Drug x Time x ISI & 27 & 1.767 & $\mathbf{0 . 0 1 2}$ \\
Drug & 3 & 19.886 & $<\mathbf{0 . 0 0 1}$ \\
Time & 1 & 14.218 & $\mathbf{0 . 0 0 3}$ \\
ISI & 1 & 32.029 & $<\mathbf{0 . 0 0 1}$ \\
Drug x Time & 3 & 0.650 & 0.558 \\
Drug x ISI & 3 & 1.753 & 0.175 \\
Time x ISI & 1 & 1.497 & 0.247 \\
Drug x Time x ISI & 3 & 0.440 & 0.726 \\
\hline
\end{tabular}

RMT=resting motor threshold; AMT=active motor threshold; I-O curve=input-output curve; SICI-ICF=short-latency intracortical inhibition and intracortical facilitation; SAI=short-interval afferent inhibition. $*$ Significant results at $\mathrm{p}<0.05$, d.f.: degrees of freedom. 


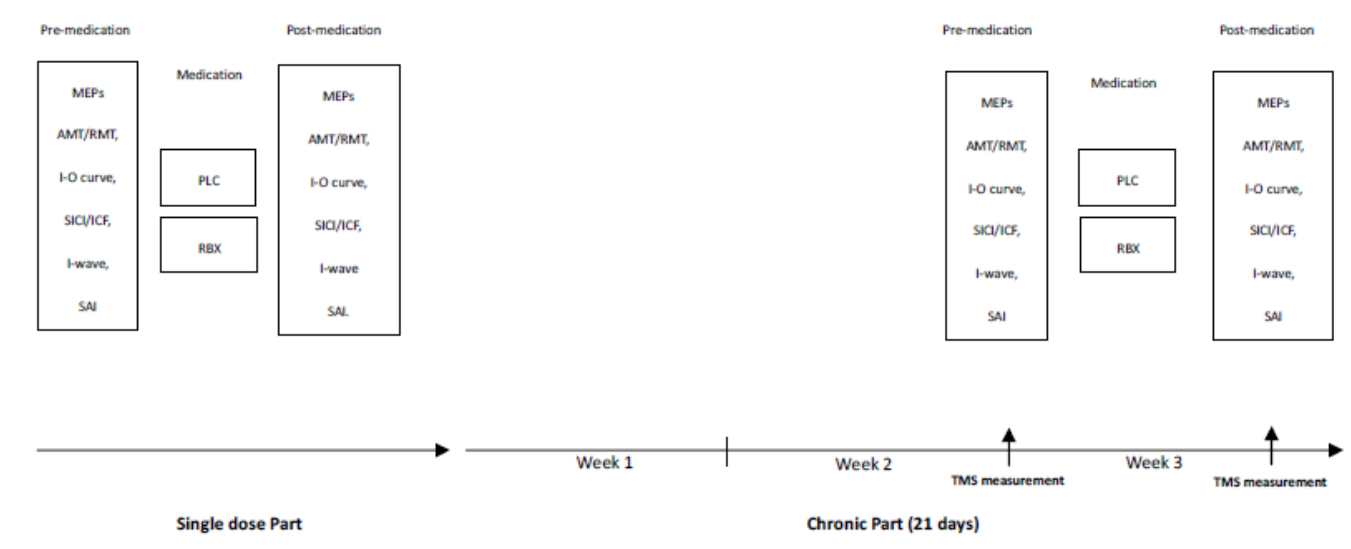

\section{Figure 1. Experimental course of the present study}

The study was conducted in two parts (single dose part and chronic part). For the single dose part, subjects received $8 \mathrm{mg}$ single dose reboxetine (sRBX) or placebo medication (sPLC). First, transcranial magnetic stimulation (TMS) was applied over the left motor cortical representation area of the right abductor digiti minimi muscle (ADM) with an intensity to elicit motor-evoked potentials (MEPs) with a peak-to-peak amplitude of on average $1 \mathrm{mV}\left(\mathrm{SI}_{1 \mathrm{mV}}\right)$. Then, active and resting motor thresholds (AMT and RMT) were determined. Afterwards, input-output curve (I-O curve), short-latency intracortical inhibition and intracortical facilitation (SICI-ICF), I-wave facilitation and short-interval afferent inhibition (SAI) were conducted in randomized order. Two hours after intake of the medication, the same TMS measurements were repeated to explore the effect RBX on excitability. For the chronic part, the same subjects took $8 \mathrm{mg}$ RBX consecutively for 21 days. During this period, the other two sessions (cRBX: with RBX at the day of experiment or cPLC: with PLC at the day of experiment) were conducted. The design of each session in the chronic part was the same as for the respective single dose condition. 
A

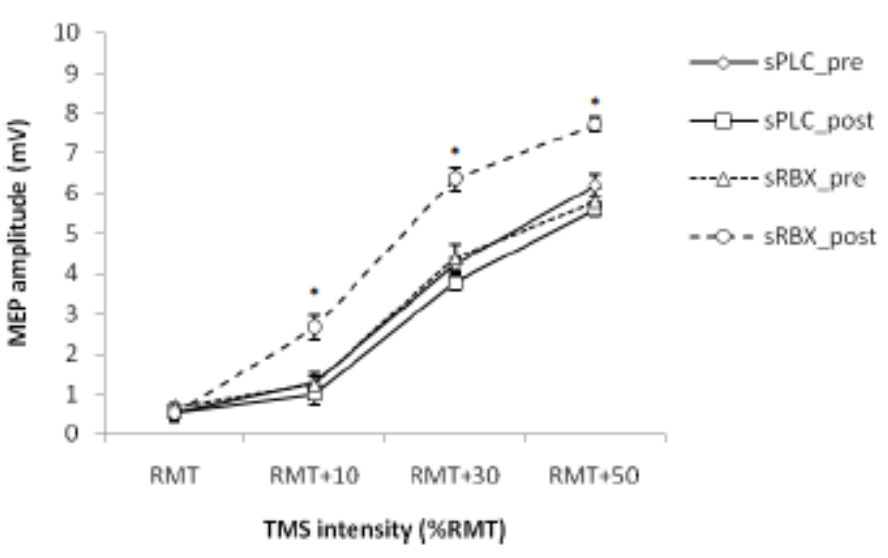

B

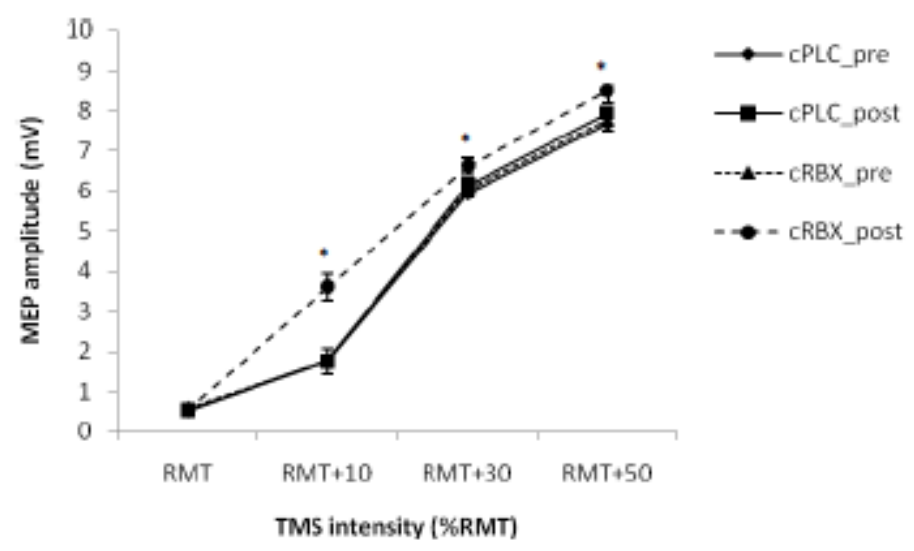



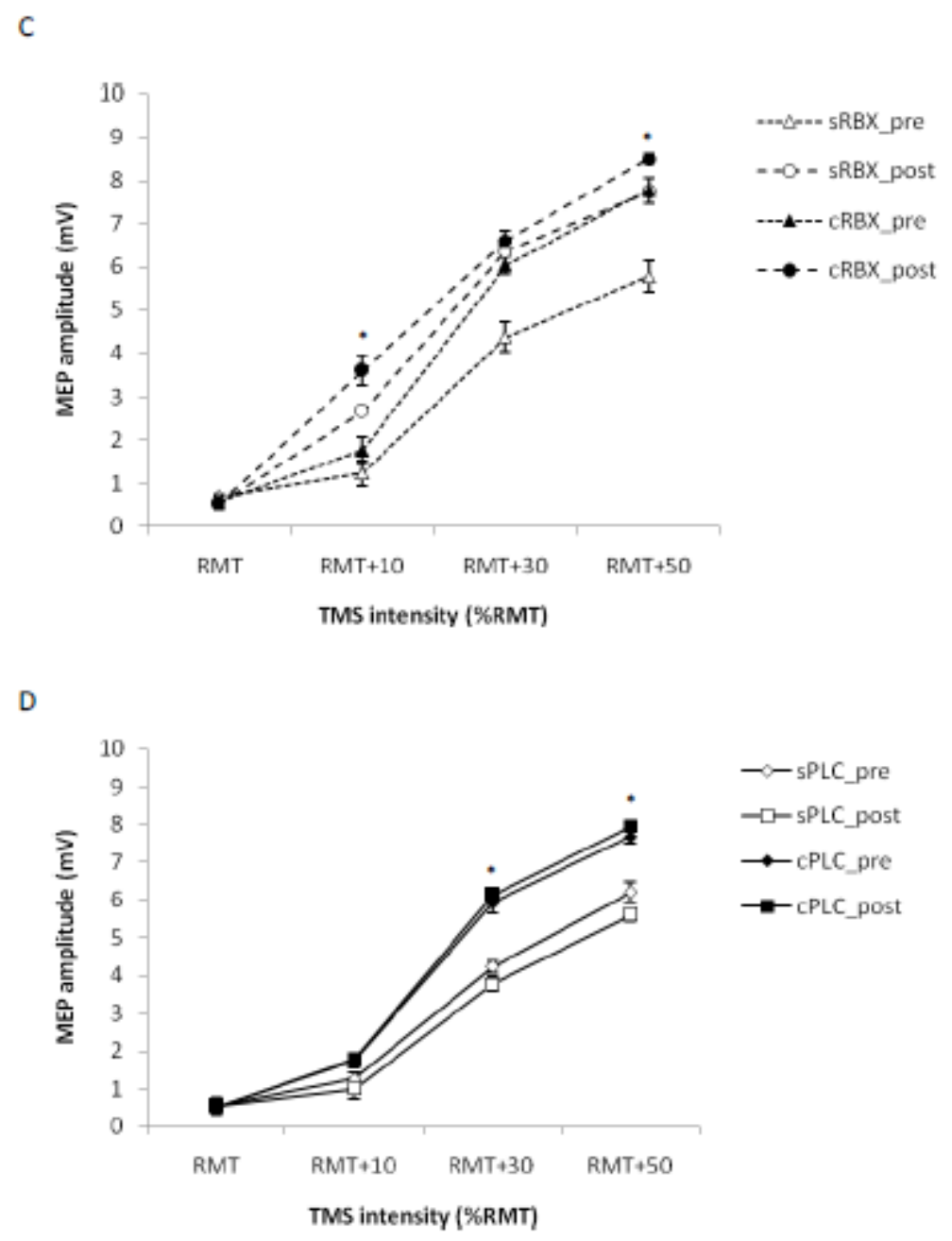

\section{Figure 2. Input-output curve values before and after drug administration}

A-D displays the MEP amplitudes (means \pm SEM) at 100, 110, 130 and $150 \%$ of resting motor threshold (RMT) under four conditions: single dose placebo (sPLC), single dose reboxetine (sRBX), chronic reboxetine+placebo medication at the day of experiment (cPLC), and chronic reboxetine+reboxetine at the day of experiment (cRBX). A: sRBX_post showed significantly larger MEP amplitudes compared to sPLC_post elicited by 110, 130, and 150\% RMT TMS. B: cRBX_post showed significantly larger MEP amplitudes compared to cPLC_post at 110, 130 and 150\% RMT TMS. C: cRBX_post significantly enhanced the MEP amplitude compared to sRBX_post at 110 and $150 \%$ RMT TMS. D: cPLC_post showed significantly enhanced MEP compared to sPLC_post at 130 and 150\% RMT TMS. Asterisks 
indicate significant differences (Student's t-test, $\mathrm{P}<0.05$ ). Vertical bars depict standard error of mean (SEM). 
A

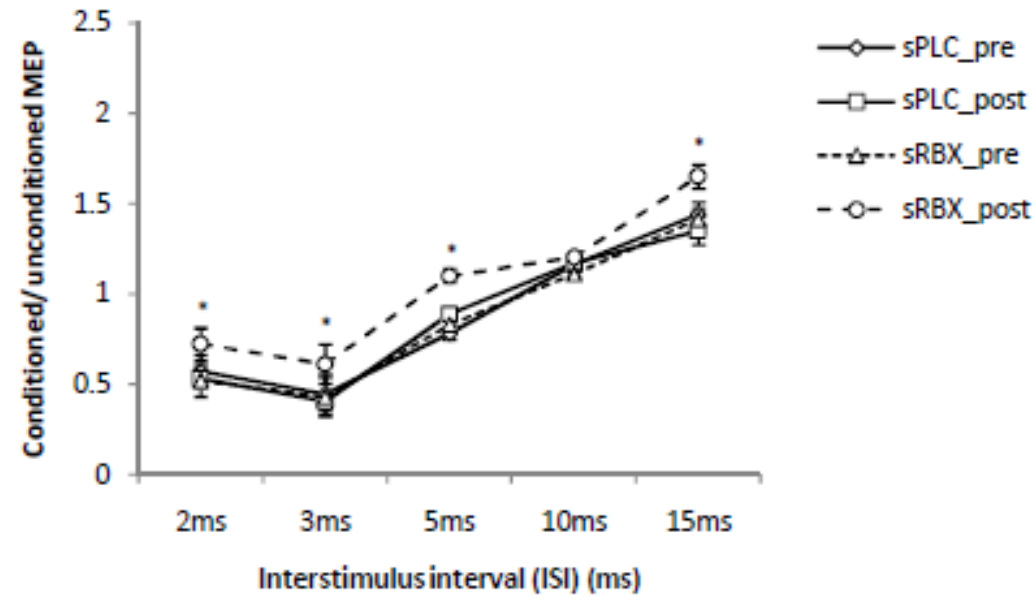

B

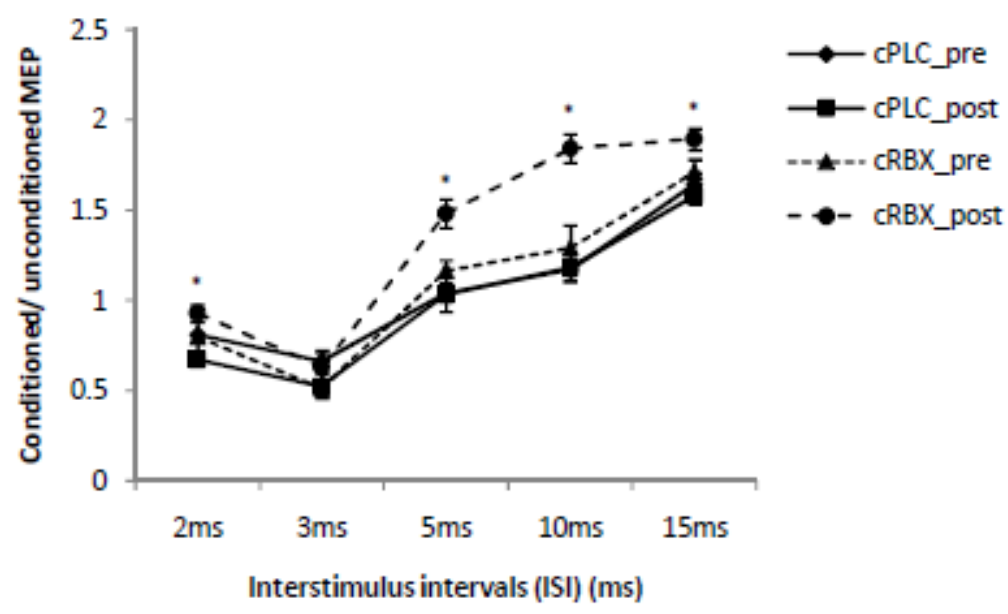


C

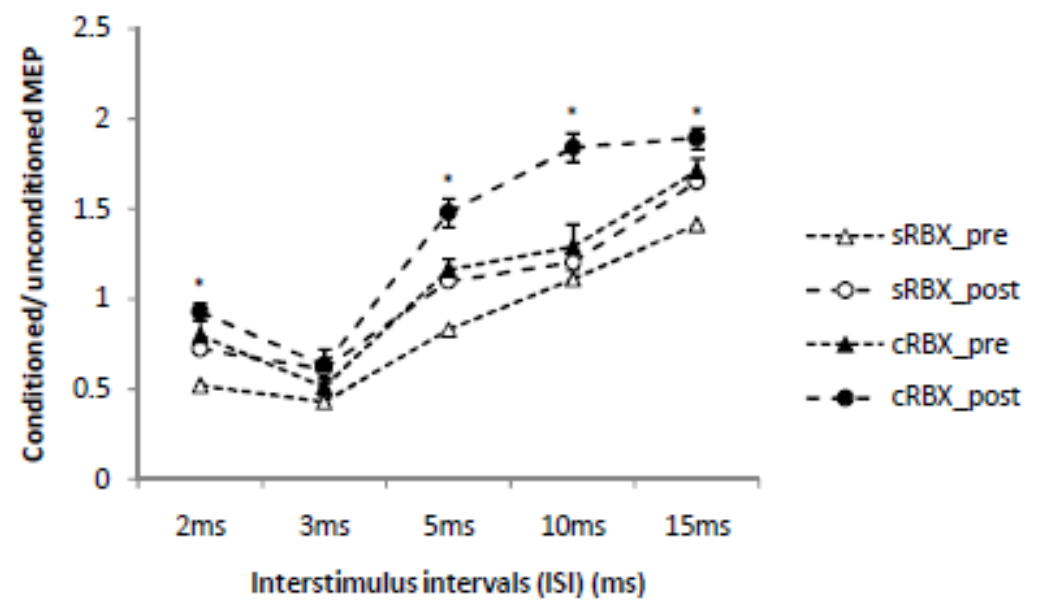

D

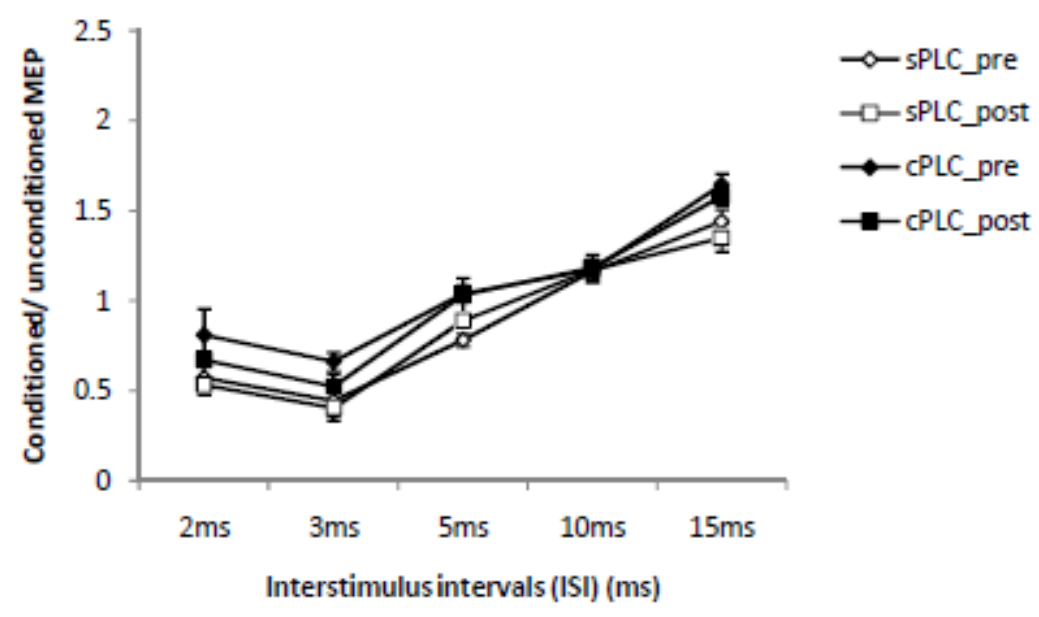

Figure 3. Short-latency intracortical inhibition and intracortical facilitation before and after drug administration

Single-pulse standardized double stimulation MEP amplitude ratios \pm SEM are depicted for ISIs revealing inhibitory (ISIs of 2, 3 and $5 \mathrm{~ms}$ ) and facilitatory (ISIs of 10 and $15 \mathrm{~ms}$ ) effects for different medication conditions: single dose placebo (sPLC), single dose RBX (sRBX), chronic reboxetine+placebo medication at the day of experiment (cPLC), and chronic reboxetine+reboxetine at the day of experiment (cRBX). A: The sRBX_post condition significantly increased facilitation for the ISI of $15 \mathrm{~ms}$ and decreased inhibition for ISIs of 2, 3 and $5 \mathrm{~ms}$ compared to sPLC_post condition. B: cRBX_post showed a significant increase of facilitation for the ISIs of 
10 and $15 \mathrm{~ms}$ and a significant decrease of inhibition for the ISI of 3 and $5 \mathrm{~ms}$ compared to cPLC_post. C: cRBX_post showed significant facilitation for the ISIs of 10 , and $15 \mathrm{~ms}$ and significant decrease of inhibition for the ISIs of 2 and $5 \mathrm{~ms}$ compared sRBX_post. D: cPLC_post showed a non-significant trend torwards enhanced facilitation and decreased inhibition compared to sPLC_post. Asterisks indicate significant differences (Student's t-test, $\mathrm{P}<0.05$ ). Vertical bars depict standard error of mean (SEM). 
A

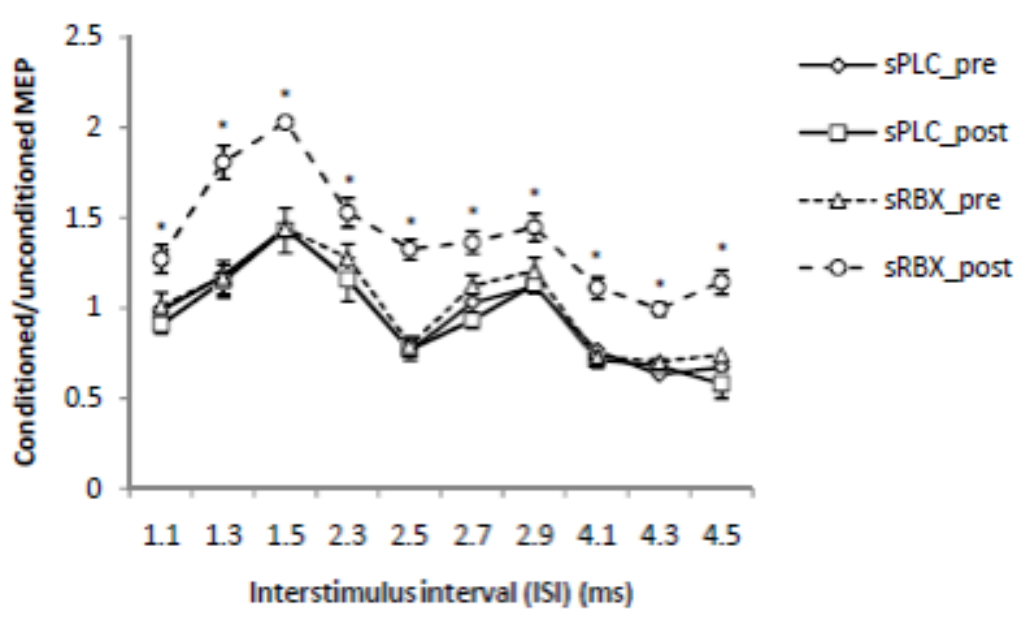

B

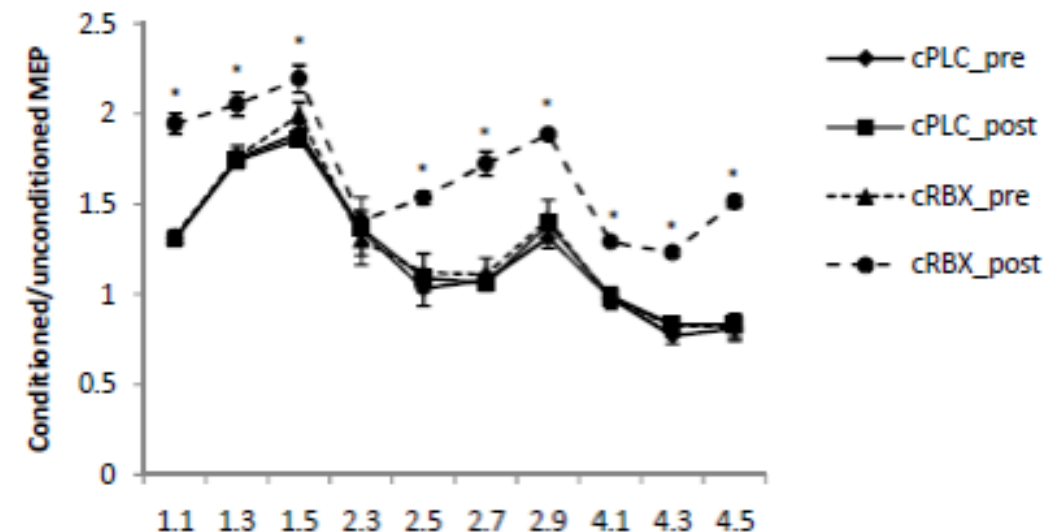

Interstimulus interval (ISI) (ms) 
C

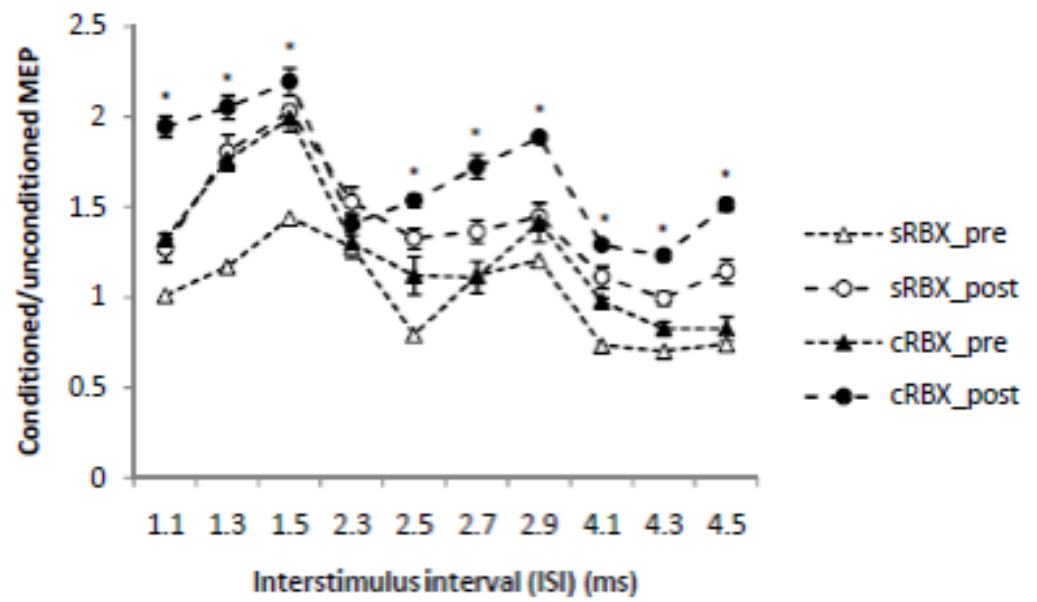

D

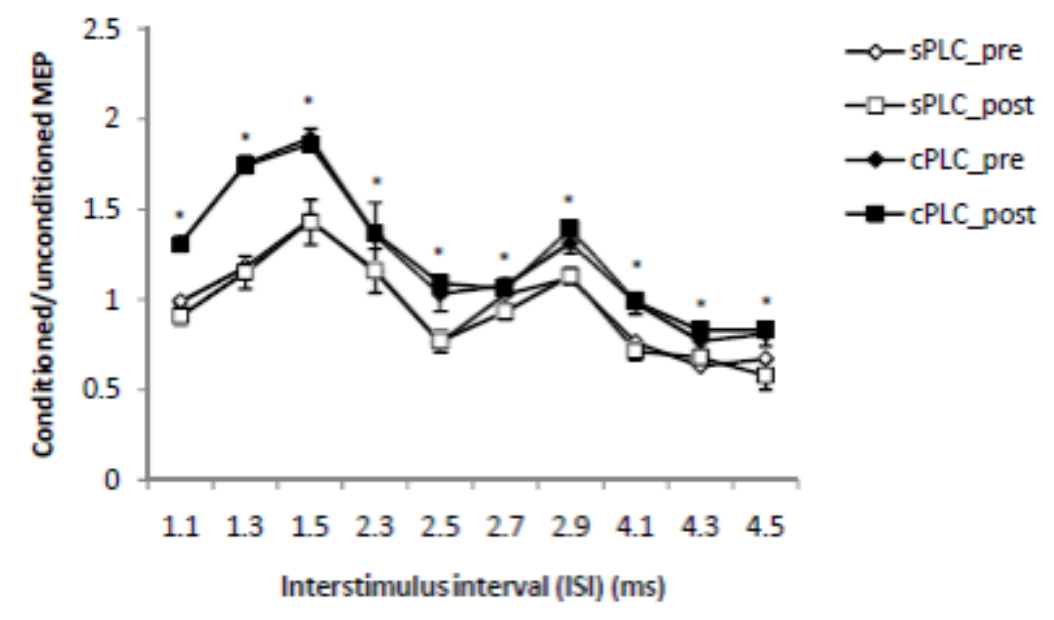

Figure 4. I-wave facilitation before and after drug administration

Single-pulse standardized double stimulation MEP amplitude ratios \pm SEM are depicted for ISIs of $1.1,1.3,1.5,2.3,2.5,2.7,2.9,4.1,4.3$ and $4.5 \mathrm{~ms}$ with different conditions: single does placebo (sPLC), single dose reboxetine (sRBX), chronic reboxetine + placebo medication at the day of experiment (cPLC), and chronic reboxetine + reboxetine at the day of experiment (cRBX). A: sRBX_post significantly enhanced MEP amplitude at all ISIs compare to sPLC_post. B: cRBX_post significantly enhanced I-wave facilitation at most ISIs compared to cPLC_post. C: cRBX_post revealed a significant enhancement of facilitation at most ISIs compared to sRBX_post. D: cPLC_post demonstrated significantly increased facilitation at all 
ISIs compared to sPLC_post. Asterisks indicate significant differences (Student's t-test, $\mathrm{P}<0.05)$. Vertical bars depict standard error of mean (SEM). 


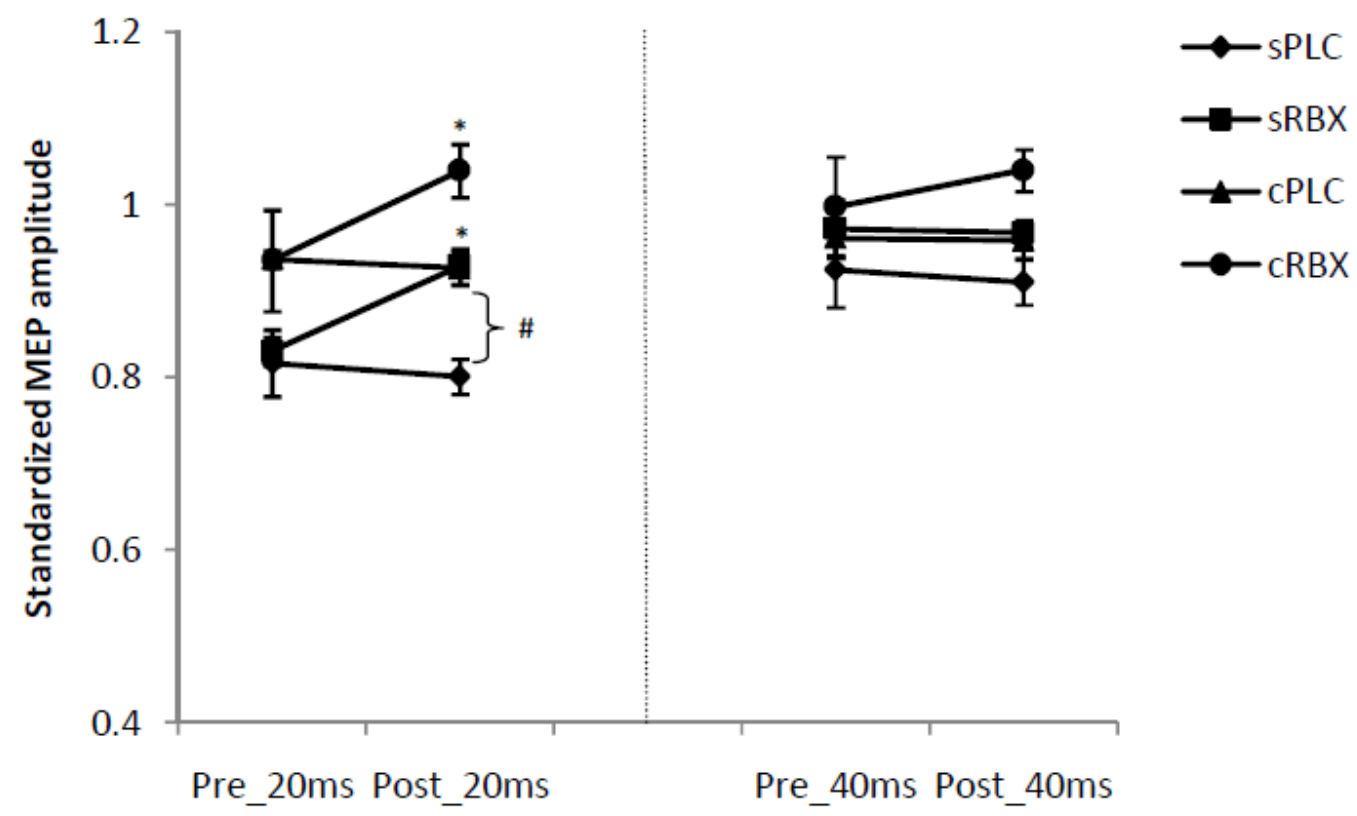

Figure 5. Short-interval afferent inhibition before and after drug administration

The figure displays SAI under different conditions: single dose placebo (sPLC), single dose reboxetine (RBX), chronic reboxetine+placebo medication at the day of experiment (cPLC), and chronic reboxetine+reboxetine at the day of experiment (cRBX). After application of both, single dose and chronic reboxetine, SAI was significantly decreased for ISI 20. As compared to the sPLC condition, in the cPLC condition, SAI was significantly reduced at ISI 20ms. Asterisks indicate significant differences of the MEP amplitude between before and after drug intake (Student's t-test, $\mathrm{P}<0.05)$. The Hash symbol indicates significant differences of the MEP amplitude between sPLC and cPLC after drug intake. Vertical bars depict standard error of mean (SEM). 


\section{Chapter 3-Summary}

\subsection{General remarks}

The studies included in the thesis explored the impact of the neuromodulators serotonin and noradrenaline on human brain physiology relevant for cognition and behavior, namely cortical excitability and plasticity. In the first study, our results showed that chronic activation of serotonergic systems enhanced and prolonged facilitatory plasticity, whereas it converted inhibitory plasticity into facilitation. In addition, serotonin modulated N-methyl-D-asparate (NMDA) receptor-dependent plasticity, but did not induce plasticity by itself. In the second study, both acute and chronic noradrenergic activation enhanced facilitatory plasticity, whereas it converted inhibitory plasticity into facilitation, but chronic application showed more stable effects. In the third study, acute and chronic enhancement of the noradrenergic system increased cortical excitability via enhancement of facilitation and reduction of inhibition. Furthermore, the chronic medication condition resulted in a more stable cortical excitability enhancement as compared to single dose application. These findings add important information to our understanding of the mechanisms of consolidation of neuroplasticity and excitability in the human cortex.

\subsection{Functional implications}

Our findings confirm that serotonin and noradrenaline impact on plasticity and excitability of the human brain. In the field of clinical application, the results might imply that serotonergic and noradrenergic agents have therapeutic effects by enhancing facilitatory plasticity and increasing cortical excitability. Moreover, since chronic administration showed more stable results, this might partially explain why 
the maximal effects of antidepressant agents are usually obtained after prolonged treatment periods.

Recently, transcranial direct current stimulation (tDCS) is increasingly applied for treatment of neurological and psychiatric diseases (Kuo et al., 2014). In many cases, it is used to induce facilitatory plasticity for therapeutic effects. Given the strengthening effect of citalopram and reboxetine on the after-effects of tDCS, combining tDCS with pharmacological agents might be a promising venue to enhance its clinical impact. In accordance, it was shown recently that combined tDCS and selective serotonin reuptake inhibitor (SSRI) treatment had a superior impact on major depression, compared both of the interventions alone or placebo treatment (Brunoni et al., 2013). Likewise, SSRIs and selective noradrenaline reuptake inhibitors (NRI) might be suited to strengthen tDCS effects in other diseases such as Alzheimer's disease, Parkinson's disease, or motor rehabilitation after stroke. Future studies should explore these possibilities and include functional outcomes to assess the agonistic effects of brain stimulation and SSRI/selective NRI therapy.

\subsection{Limitations}

Some potential limitations of the present work should be taken into account. First, we did not obtain drug plasma levels, which would have enabled exploration of dosage-dependent effects of the medication to some extent. Moreover, all studies in the thesis were conducted in healthy subjects. In neuropsychiatric diseases, transmitter availability and other features of brain function might be different. Finally, due to the limited time frame, we did not have the chance to explore the behavioral effects of serotonin and noradrenaline in these studies, thus presumed functional implications are speculative at present. 


\subsection{Future perspectives}

Our studies explored the impact of serotonergic activation on neuroplasticity and noradrenergic activation on plasticity as well as on cortical excitability in humans. The results supply clear evidence for the relevance of these receptors with regard to respective physiological processes. Future studies should explore the mechanisms of action of these effects in larger detail, regarding dosages, receptor subtypes, and different plasticity induction techniques. Apart from experiments in humans, cellular, slice and in vivo animal experiments are essential, and will help to understand the mechanisms of action of both neuromodulators on plasticity and excitability into larger detail, because they enable the exploration of biological effects on a level not accessible for experiments in humans.

Pathologically altered plasticity, especially compromised LTP, recently came into the focus of attention as a potentially important pathophysiological mechanism in various neurological and psychiatric diseases, e.g., major depression, Alzheimer's disease, Parkinson's disease, and others. However, our study was conducted in healthy young humans. A one-to-one transferability of the results to participants with other characteristics cannot be taken for granted, because the basal state of brain activity and excitability will differ between groups, which might relevantly affect intervention effects. For future studies, it will be interesting to explore if therapeutic medication in these patients improves symptoms at least partly due to a re-establishment of plasticity or pathologically altered excitability.

Beyond tDCS, a variety of stimulation methods with different plasticity mechanisms is available that can be used to evaluate the effects of neuromodulatory systems on plasticity in humans. For example, PAS shows more focal effects 
compared to tDCS. Furthermore, similar to other neuromodulators such as dopamine, the effects of SSRI and selective NRI might be specific for the kind of plasticity induction (Kuo et al., 2008; Fresnoza et al., 2014 ). Exploration of the intervention on different cortical areas could also be interesting in the future, since effects might differ due to area-specific receptor subtype composition. Specifically, plasticity induction of the dorsolateral prefrontal cortex with tDCS could be relevant for our understanding of the pathophysiological foundation of depression (Rajji et al., 2013), because this cortical area is closer related to respective symptoms of the disease as compared with the primary motor cortex, which we investigated in our present study.

Neuroplasticity and cortical excitability are thought to be important foundations for cognition and motor learning. Serotonergic and noradrenergic agents have been shown to improve cognition and motor behavior in healthy humans and patients suffering from neurological and psychiatric disorders (Loubinoux et al., 2002; Ferguson et al., 2003). In addition, for acute and chronic administration of the drug, this functional improvement was accompanied by enhanced cortical excitability and also facilitatory plasticity (Pariente et al., 2001; Loubinoux et al., 2002). It is important to investigate further if alterations of plasticity and cortical excitability induced by serotonergic and noradrenergic agents are related to the respective cognitive effects and functional outcomes. Future studies will explore the association between neuroplasticity, cortical excitability, cognitive performance, and functional outcome in larger detail.

Overall, improved knowledge about the mechanisms of neuroplasticity and excitability of the human brain will strengthen the possibility to shape the plastic potential of the brain, and might open a broader field of new therapeutic and research perspectives. However, we are still at the beginning of our understanding of the 
neurophysiological and functional effects of neuromodulators on the human central nervous system. The central involvement of these substances in various brain functions in health and disease makes related studies important for improving our understanding of brain functions, but also for development of new therapeutic strategies to treat people suffering from diseases involving pathological alterations of neuromodulatory activity. 


\section{References}

Abbruzzese G \& Trompetto C. (2002). Clinical and research methods for evaluating cortical excitability. J Clin Neurophysiol19, 307-321.

Aydina K, Ucarb A, Oguzc K, Okurd O, Agayevb A, Unal Z \& et al. (2007). Increased Gray Matter Density in the Parietal Cortex of Mathematicians: A Voxel-Based Morphometry Study. Am J Neuroradiol28, 1859-1864.

Balzarotti S \& Colombo B. (2016). Effects of unilateral transcranial direct current stimulation of left prefrontal cortex on processing and memory of emotional visual stimuli. PLoS One19, 11.

Barker A, Jalinous R \& Freeston I. (1985). Non-invasive magnetic stimulation of human motor cortex. Lancet1, 1106-1107.

Batsikadze G, Paulus W, Kuo MF \& Nitsche MA. (2013). Effect of serotonin on paired associative stimulation-induced plasticity in the human motor cortex. Neuropsychopharmacology38, 2260-2267.

Bezchilbnyk-Butler K, Aleksic I \& Kennedy S. (2000). Citalopram- a review pf pharmachological and clinical effects. J Psychiatry Neurosci25, 241-254.

Bhagya V, Srikumar B, Traju T \& Rao B. (2015). The selective noradrenergic reuptake inhibitor reboxetine restores spatial learning deficits, biochemical changes, and hippocampal synaptic plasticity in an animal model of depression. Jounal of Neuroscience Research93, 104-120.

Bindman L, Lippold O \& Redfearn J. (1964). The action of brief polarizing currents on the cerebral cortex of the rat (1) during current flow and (2) in the production of long-lasting after-effects. J Physiol172, 369-382.

Boroojerdi V, Battaglia F, Meullenbacher W \& Cohen L. (1999). Evaluation of the effects of CNS-active drugs on cortical excitability in intact humans. Neurology 52, 457.

Brunoni A, Nitsche MA, Bolognini N, Bikson M, Wagner T \& Merabet L. (2012). Clinical research with transcrnial direct current stimulation (tDCS): challenges and future directions. Brain Stimul5, 175-195.

Brunoni A, Valiengo L, Baccaro A, Zanao T, Oliveira JD \& Goulart A. (2013). The sertraline vs electrical current therapy for treating depression clinical study: results from a factorial, randomized, controlled trial. JAMA Psychiatry70, 1-9.

Buonomano D \& Merzenich M. (1998). Cortical plasticity: from synapses to maps. Annu Rev Neurosci21, 149-186.

Campell S \& Macqueen G. (2004). The role of the hippocampus in the pathophysiology of major depression. J Psychiatry Neurosci29, 417-426. 
Castren E. (2004). Neurotrophic effects of antidepressant drugs. Curr Opin Pharmacol4, 58-64.

Chen R. (2000). Studues of human motor physiology with transcrnial magnetic stimulation. Muscle Nerve Suppl9, 26-32.

Cooke S \& Bliss T. (2006). Plasticity in the human central nervous system. Brain 129, 1659-1657.

Coppell A, Pei Q \& Zetterström T. (2003). Bi-phasic change in BDNF gene expression following antidepressant drug treatment. Neuropharmachology44, 903-910.

Criti A \& Malenka R. (2008). Synaptic plasticity: multiple foms, functions, and mechanisms. Neuropsychopharmachology33, 18-41.

Di LazzoroV, Oliviero A, profice P, Pennis MA, Pilato F, Zito G \& et al. (2003). Ketamine increases human motor cortex excitabilty to transcranial magnetic stimulation. J Physiol547, 485-496.

Di Lazzoro V, Pilato F, Dilenone M, Saturno E, Oliviero A, Marra C \& et al. (2006). In vivo cholinergic circuit evaluation in frontotemporal and Alzheimer dementias. Neuroplogy66,1111-1113.

Ferguson J, Wesnes K \& Schwartz G. (2003). Reboxetine versus paroxetine versus placebo: effects on conitive functioning in depressed patients. International Clinical Psychopharmacology18, 9-14.

Fresnoza S, Stiksrud E, F Klinker, D Liebetanz, W Paulus, MF Kuo \& Nitsche. MA. (2014). Dosage-dependent effect of dopamine D2 receptor activation on motor cortex plasticity in humans. J Neurosci34, 10701-10709.

Gartside I. (1968). Mechanisms of sustained increases of firing rate of neurones in the rat cerebral cortex after polarization: role of protein synthesis. Nature 26, 383-384.

Gerdelat-Mas A, Loubinoux I, Tombari D, Rascol O, Chollet F \& Simonetta-Moreau M. (2005). Chronic administration of selective serotonin reuptake inhibitor (SSRI) paroxetine modulates human motor cortex excitability in healthy subjects. Neuroimage27, 314-322.

$\mathrm{Gu}$ Q. (2002). Neuromodulatory transmitter systems in the cortex and their role cortical plasticity. Neuroscience111, 815-835.

Hass H \& Konnerth A. (1983). Histamine and noradrenaline decrease calcium-activated potasium conductance in hippocampal pyramidal cells. Nature302, 432-434.

Hattori Y, Moriwaki A \& Hori Y. (1990). Biphasic effects of polarizing current on 
adenosine-sensitive generation of cyclic AMP in rat cerebral cortex. Neuroscience Letters 116, 320-324.

Heinbotham L \& Dunwiddie T. (1991). Long-term increases in the evoked population spike in the CA1 region of rat hippocampal pyramidal cells. Nature302, 432-434.

Henn F \& Vollmayer B. (2004). Basic pathophysiological machanisms in depression: what are they and how might they affect the course of the illness? . Pharmacopsychiatry 37, 152-156.

Herwig U, Brauer K, Connemann B, Spitzer M \& Schonfeldt-Lecuona C. (2002). Intracortical excitability is modulated by a norepinephrine-reuptake inhibitor sa measured with piared-pulse transcrnial magnetic stimulation. Psychopharmacology164, 228-232.

Hodics T, Cohen LC \& Cramer S. (2006). Functional imaging of intervention effects in stroke motor rehabilitation. Arch Phys Med Rehabil 87, 36-42.

Hu H, Real E, Takamiya K, Kang M, Ledoux J, Huganir RL \& et al. (2007). Emotion enhances learning via norepinephrine regulation of AMPA-receptor trafficking. Cell131, 160-173.

Islam N, Aftabuddin M, Moriwaki A, Hattori Y \& Hori Y. (1995a). Increase in the calcium level following anodal polarization in the rat brain. Brain Res684, 206-208.

Islam N, Moriwaki A \& Hori Y. (1995b). Co-localization of c-fos protein and protein kinase $\mathrm{C}$ gamma in the rat brain following anodal polarization. Indian $J$ Physiol Pharmacol39, 209-215.

Johansen-Berg H, Scholz J \& Stagg C. (2010). Relevance of structural brain connectivity to learning and recovery from stroke. Front Syst Neurosci2, 1-9.

Kasper S, Giamal N \& Hilger E. (2000). Reboxetine: the first selective noradrenaline re-uptake inhibitor. Exp Opin Pharmacother1, 771-782.

Katsuki H, Izumi Y \& Zorumski C. (1997). Nordrenergic regulation of synaptic plasticity in the hippocampal CA1 region. Jounal of Neurophysiology77, 3013-3020.

Kemp A \& Manahan-Vaughan D. (2005). The 5-hydroxytryptamine4 receptor exhibits frequnecy-dependent properties in synaptic plasticity and behavioral metaplasticity in the hippocampal CA1 region in vivo. Cereb Cortex 15, 1037-1043.

Kemp A \& Manahan-Vaughan D. (2008). Beta-adrenoreceptors comprise a critical element in learning-facilitated long-term platicity. Cerebral Cortex18, 1326-1334. 
Kirwood A, Rozas C, Kirwood J, Perez F \& Bert M. (1999). Mosulation of long-term synaptic depression in visual cortex by acetylcholine and norepinephrine. $J$ Neurosci19, 1599-1609.

Kobayashi M \& Pascual-Leone A. (2003). Transcranial magnetic stimulation in neurology. Lancet Neurol2, 145-156.

Kojic L, Gu Q, Douglas R \& Cynader M. (1997). Serotonin facilitates synaptic plasticity in kitten visual cortex: an in vitro study. Brain Res DEv Brain Res101, 299-304.

Korchounov A, Ilic T \& Ziemann U. (2003). The alpha 2-adrenergic agonist guanfacine reduces excitability of human motor cortex through disfacilitation and increase of inhibition. Clinical Neurophysiol114, 1834-1840.

Korchounov A \& Ziemann U. (2011). Neuromodulatory neurotransmitters influence LTP-like plasticity in human cortex: a pharmaco-TMS study. Neuropsychopharmachology36, 1894-1902.

Kuo HI, Paulus W, Batsikadze G, Jamil A, Kuo MF \& Nitsche MA. (2016). Chronic enhancement of serotonin facilitatory excitatory transcrnial direct current-induced plasticity. Neuropsychopharmachology41, 1223-1230.

Kuo MF, Paulus W \& Nitsche MA. (2008). Boosting focally-induced brain plasticity by dopamine. Cereb Cortex 18, 648-651.

Kuo MF, Paulus W \& Nitsche MA. (2014). Therapeutic effects of non-invasive brain stimulation with direct currents (tDCS) in neuropsychiatric diseases. Neuroimage 15, 948-960.

Lapiz M, Bondi C \& Morilak D. (2007). Chronic treatment with desipramine improves cognitive performance of rats in an attentional set-shifting test. Neuropharmachology32, 1000-1010.

Lei S, Deng P, Porter J \& Shin H. (2007). Adrenergic facilitation of GABAergic transmission in rat entorhinal cortex. J Neurophysiol98, 1868-1877.

Levy C, Nichols D, Schmalbrock P, Keller P \& Chakeres D. (2001). Functional MRI evidence of cortical reorganization in upper-Limb stroke hemiplegia treated with constraint-induced movement therapy. Am J Phys Med Rehabil80, 4-12.

Liebtanz D, Nitsche M, Tergau F \& Paulus W. (2002). Pharmacological approach to synaptic and membrane mechanisms of DC-induced neuroplasticity in man. Brain 125, 2238-2247.

Lisman J. (2001). Three Ca2+ levels affect plasticity differently: the LTP zone, the LTD zone and no man's land. $J$ Physiol532, 285.

Loubinoux I, Pariente J, Boulanouar K, Carel C, Manelfe C, Rascol O \& et al. (2002). A single dose of the serotonin neurotransmission agonist paroxetine enhnaces 
motor output: Double-blinded, placebo-controlled, fMRI study in healthy subjects. Neuroimage15, 26-36.

Loubinoux I, Tombari D, Parente J, Gerdela-Mas A, Franceries X, Cassol E \& et al. (2005). Modulation of behavior and cortical motor activity in healthy subjects by a chronic administration of a serotonin enhancer. Neuroimage27, 299-313.

Malenka R \& Bear MF. (2004). LTP and LTD: an embarrassment of riches. Neuron 441, 5-21.

Martin S, Grimwood P \& Morris R. (2000). Synaptic plasticity and memory: an evaluation of the hypothesis. Annu Rev Neurosci23, 649-711.

Marzo A, Bai J \& Otani S. (2009). Neuroplasticity regulation by noradrenaline in mammalian brain. Current Neuropharmacology7, 286-295.

Merton P \& Morton H. (1980). Stimulation of the cerebral cortex in the intact human subject. Nature 285, 227.

Nakadate K, Matsukawa M \& Okado N. (2006). Identification of adrenoreceptor subtype-medisted changes in the density of synapses in the rat visual cortex Neuroscience 138, 37-46.

Nitsche MA, Grundey J, Liebetanz D, Lang N, Tergau F \& Paulus W. (2004). Catecholaminergic consolidation of motor cortical neuroplasticity in humans. Cereb Cortex 14, 1240-1245.

Nitsche MA, Kuo MF, Karrasch R, Warden B, Liebtanz D \& Paulus W. (2009). Serotonin affects transcrnial direct current (tDCS)-induced neuroplasticity in humans. Biol Psychiatry66, 503-508.

Nitsche MA, Cohen LG, Wassermann EM, Priori A, Lang N, Antal A \& et al. (2008). Transcranial direct surrent stimulation: state of art 2008. Brain Stimul1, 206-223.

Nitsche MA, Fricke K, Henschke U, Schlitterlau A, Liebtanz D, Lang N \& et al. (2003b). Pharmacological modulation of cortical excitability shifts induced by transcrnial DC stimulation. J Physiol533, 293-301.

Nitsche MA, Klein C, Tergau F, Rothwell J \& Paulus W. (2003a). Level of action of cathodal DC polarization induced inhibition of the human motor cortex. Clin Neurophysiol144, 600-604.

Nitsche MA, Muller-Dahlhaus F, Paulus W \& Ziemann U. (2012). The pharmacology of neuroplasticity induced by non-invasive brain stimulation: building models for the clinical use of CNS active drugs. J Physiol590, 4641-4662.

Nitsche MA \& Paulus W. (2000). Excitability changes induced in the human motor cortex by weak transcranial direct current stimulation. $J$ Physiol527, 633-639. 
Nitsche MA \& Paulus W. (2001). Sustained excitability elevations induced by transcranial DC motor cortex stimulation in humans. . Neurology57, 1899-1901.

Normann C, Schmitz D, Furmaier A, Doing C \& Bach M. (2007). Long-term platsicity of visually evoked potentials in humans is altered in major depression. Biol Psychiatry62, 373-380.

Ohashi S, Machiko M, Togashi H, Ueno K \& Yoshioka M. (2003). The serotonergic modulation of synaptic plasticity in the rat hippocmapo-medial prefrontal cortex pathway. Neuroscience letters342, 179-182.

Ohashi S, Matsumoto M, Otani H, Mori K, Togashi H \& Ueno K. (2002). Changes in synaptic plasticity in the rat hippocmapo-medial prefrontal cortex pathway induced by repeated treatment with flouxamin. Brain Res949, 131-138.

Page M \& Lucki I. (2002). Effects of acute and chronic reboxetine treatment on stress-induced manoamine efflux in the rat frontal cortex. Neuropsychopharmachology27, 238-247.

Pantev C, Roberts L, Schulz M, Engelien A \& Ross B. (2001). Timbre-specific enhancement of auditory cortical representations in musicians. Neuroreport 12, 169-174.

Pariente J, Loubinoux I, Carel C, Albucher J, Leger A, Manelfe C \& et al. (2001). Flouxetine modulates motor performance and cerebral activation of patients recovering from stroke. Ann Neurol50, 718-729.

Park I, Lee K, Han J, Lee NJ, Lee W, Park KA \& et al. (2009). Experience-dependent plasticity of cerebellar vermis in basketball players. Cerebellum8, 334-339.

Park S, Jang H, Cho K, Kim M, Yoon S \& Rhie D. (2012). Developmental switch of the serotonergic role in the induction of synaptic long-term potentiarion in the rat visual cortex. Korean J Physiol Pharmacol16, 65-70.

Plewnia C, Hoppe J, Cohen L \& Gerloff C. (2004). Improved motor skill acquisition after selective stimulation of central norepinephrine. Neurology62, 2124-2126.

Plewnia C, Hoppe J, Heimke C, Bartles M, Cohen L \& Gerloff C. (2002). Enhancement of human cortico-motoneuronal excitability by the selective norepinephrine reuptake inhibitor reboxetine. Neuroscience letters330, 231-234.

Purpura D \& Mcmurtry J. (1965). Intracellular activities and evoked potential changes during polarization of motor cortex. J Neurophysiol 28, 166-185.

Rajji T, Sun Y, Zomorrodi-Moghaddam R, Farzan F, Blumberger D, Mulsant BH \& et al. (2013). PAS-induced potentiation of cortical evoked activity in the dorsolateral prefrontal Cortex. Neuropsychopharmachology38, 2545-2552. 
Rioult-Pedotti M, D DF \& Donoghue J. (2000). Learning-induced LTP in neocortex. Science290, 533-536.

Robinson E. (2012). Blockade of noradrenaline re-uptake improves accuracy and impulse control in rat performing a five-choixe serial reaction time tasks. Psychopharmacology219, 303-312.

Rothwell J. (1993). Evoked potentials, magnetic stimulation studies, and event-related potentials. Curr Opin Neurol6, 715-723.

Shin Y, Foerster A \& Nitsche MA. (2015). Transcrnial direct current stimulation (tDCS)-application in neuropsychology. Neuropsychologia69, 154-175.

Straube T \& Frey J. (2003). Involvement of beta-adrenergic receptors in protein synthesis-dependent late long-term potentiation (LTP) in the dentate gyrus of freely moving rats: the critical role of the LTP induction strength. Neuroscience119, 473-479.

Ueki Y, Mima T, Kotb M, Sawada H, Saiki H, Ikeda A \& et al. (2006). Altered plasticity of the human motor cortex in Parkinson's disease. Ann Neurol59, 60-71.

Wagner T, Valero-Cabre A \& Pascual-Leone A. (2007). Noninvasive human brain stimulation. Annu Rev Biomed Eng9, 527-565.

Wang L, Fink G, Dafotakis M \& Grefkes C. (2009). Noradrenergic stimulation and motor performance: different effects of reboxetine on movement kinetics and visuiomotor abilities in healthy human subjects. Neuropsychologia47, $1302-1312$.

Wang L, Fink G, Diekhoff S, Rehme A, Eickoff S \& Grefke C. (2011). Noradrenergic enhancement improves motor network connectivity in stroke patients. ANNALS of Neurology69, 375-388.

Wojtowicz A, Fidzinski P, Heinemann U \& Behr J. (2010). Beta-adrenergic receptor activation induces long-lasting potentiation in burst-spiking but not regular-spiking cells at CA1-aubiculum synapses. Neuroscience171, 367-372.

Ziemann U. (2003). Pharmacology of TMS. Suppl Clin Neurophysiol56, 226-231.

Ziemann U. (2004). TMS induces plasticity in human cortex. Rev Neurosci15, 253-266.

Ziemann U, Chen R, Cohen L \& Hallet M. (1998). Dextromethorphane decreases the excitability of the human motor cortex. Neurology51, 1320-1324.

Ziemann U, Loennecker S, Steinhoff B \& Paulus W. (1996). Effects of antiepileptic drugs on motor cortex excitability in humans: a transcrnial magnetic stimulation study. Ann Neurol40, 367-378. 
Ziemann U, Muellbacher W, Hallet M \& Cohen LG. (2001). Modulation of practice-dependent plasticity in human motor cortex. Brain124, 1171-1181.

Ziemann U, Paulus W, Nitsche M, Pascual-Leone A, Byblow W, Berardelli A \& et al. (2008). Consesus: motor cortex plasticity protocols. Brain Stimul1, 164-182.

Ziemann U, Tergau F, Wassermann E, Wischer S, Hildebrandt J \& Paulus W. (1998b). Demonstartion of facilitatory I wave interaction in the human motor cortex by paired transcranial magnetic stimulation. J Physiol511, 181-190. 


\section{Acknowledgements}

First of all, I would like to express deeply appreciation to my supervisor Prof. Michael Nitsche. The thesis would have not been possible to accomplish without his innovative ideas and encouragement. I learned so much from him during my doctoral studies. I want to thank him for his trust in the beginning and always warm supports whenever I met difficulties. His enthusiastic spirit to research and humorous attitude to life are the characters I admire the most. Hope I can keep and follow his positive and healthy attitude in my following research. Thank you to the other members of my thesis committee, Prof. Andree Niklas and Prof. Siegmar Blumentritt, for their support and brilliant advice during my $\mathrm{PhD}$.

Sincerely thank you to the chairman of the department, Prof. Walter Paulus, for his guidance and support through these years. I am also grateful to the other professors on the department: Prof. Martin Sommer, Prof. David Liebtanz, and Prof. Andrea Antal, for their help and support. Special thanks to Ms. Marion Kurze for her kind help and smiling face. To Manuel Hewitt, thank you for your professional technical help.

Lots of thanks to my former colleagues and friends from whom I learned a lot: Min-Fang Kuo, Shane Fresnoza, and Giorgi Batsikadze. Thank you to my sweet working team: Asif Jamil, Yuichiro Shirota, and Aguida Foerster for their friendship and help.

Warmest thanks to all my family members, without their support and love I could not finish the whole study. Especially to my mom, my dad, my husband, and my little boy, thank you for your endless love and support.

Finally, I would like to thank all the volunteers on the studies. Wish the studies we have done together can really benefit the people who are suffering from diseases, and make the world more wonderful in the future. 


\section{Curriculum Vitae}

Name: Kuo,Hsiao-I

Dateof Birth: Jun. 13. 1986

Nationality: Taiwan

Contact Details: Klinik fur Klinische Neurophysiologie, Robert-Koch-Str. 40

Goettingen, 37075 Germany

$(+49) 176-32983382$

Catecean20@gmail.com

\section{Education}

October 2013-present

Student, Doctor of Philosophy

University of Goettingen

Goettingen, Germany

Thesis: Effects of Serotonin and Noradrenaline on Neuroplasticity of The Primary

Motor Cortex in Humans

September 2008-June 2010

Master of Science in Physical Therapy

National Taiwan University

Taipei, Taiwan

\section{September 2004-June 2008}

Bachelor of Science in Physical Therapy

National Taiwan University

Taipei, Taiwan

\section{Employment History}

\section{2-2013}

Research assistant

National Taiwan Science Council

Taipei, Taiwan

\section{0-2012}

Physical Therapist

Department of Rehabilitation, National Taiwan University Hospital

Taipei, Taiwan

\section{8-2010}

Teaching assistant

Department of Physical Therapy, National Taiwan Univeristy

Taipei, Taiwan 


\section{Lists of Publications}

Kuo HI, Wu YT, Luo HJ, Chen PS, Jeng SF. (2009) General movement assessment for high risk infants. FJPT 34:9-17.

Kuo HI, Bikson M, Abhishek D, Minhas P, Paulus W, Kuo MF, Nitsche MA. (2013) Comparing cortical plasticity induced by conventional and high-definition $4 \times 1$ ring tDCS: a neurophysiological study. Brain Stimulation 6:644-648.

Kuo HI, Paulus W, Batsikadze G, Jamil A, Kuo MF, Nitsche MA. (2016) Chronic enhancement of serotonin facilitates excitatory transcranial dorect current stimulation-induced neuroplasticity. Neuropsychopharmacology 41:1223-1230.

Kuo HI, Paulus W, Batsikadze G, Jamil A, Kuo MF, Nitsche MA. (2016) Acute and chronic effects of noradrenergic enhancement on transcranial direct current stimulation (tDCS)-induced plasticity in humans. The Journal of Physiology (Accepted)

Kuo HI, Paulus W, Batsikadze G, Jamil A, Kuo MF, Nitsche MA. Acute and chronic noradrenergic effects on cortical excitability in healthy humans. (submitted)

Jamil A, Batsikadze G, Kuo HI, Labruna L, Hasan A, Paulus W, Nitsche MA. (2016) Systematic evaluation of the impact of stimulation intensity on neuroplastic after-effects induced by transcranial direct current stimulation. The Journal of Physiology (Accepted)

\section{Conference Presentations}

\section{September 29-October 3, 2015}

The $15^{\text {th }}$ European Congress on Clinical Neurophysiology.

Brno, Czech Republic

Poster: Chronic enhancement of serotonin facilitates excitatory transcranial direct current stimulation-induced neuroplasticity

\section{October 10-13, 2011}

The $11^{\text {th }}$ International Congress of the Asian Confederation for Physical Therapy. Bali, Indonesia

Poster: The reliability, validity, and responsiveness of the fall risk assessment tool in subjects with stroke.

\section{November 11-14, 2009}

The $60^{\text {th }}$ Scientific Conference of the Physical Therapy Association of the Republic of China.

Taipei, Taiwan

Oral presentation: Effects of the telerehabilitation on balance in subjects with with subacute stroke: a pilot study.

\section{April 4-6, 2009}


The $58^{\text {th }}$ Scientific Conference of the Physical Therapy Association Of the Republic of China.

Taipei, Taiwan

Oral Presentation: Comparison of the center of pressure during voluntary weight shift with and without virtual reality system.

\section{Awards and Scholarships}

\section{3-2015}

Government scholarship, Taiwan

\section{0}

Graduate Student Research and Development Award,

National Taiwan University, Taiwan

\section{8}

President Award

National Taiwan University, Taiwan 